\title{
Summary of Floods in the United States during 1968
}

GEOLOGICALSURVEY WATER-SUPPLY PAPER $1970-B$

Prepared in cooperution with Federul, State, and local agencies

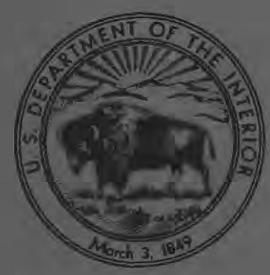




\section{Summary of Floods in the \\ United States during 1968}

By J. O. ROSTVEDT and others

FLOODS OF 1968 I N THE U N I T E D S T A T E S

GEOIOGICALSURVEY WATER-SUPPLY PAPER 1970-B

Prepared in cooperation with Federal, State, and local agencies

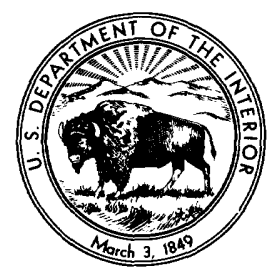


UNITED STATES DEPARTMENT OF THE INTERIOR

ROGERS C. B. MORTON, Secretary

GEOLOGICAL SURVEY

V. E. McKELVEY, Director

Library of Congress catalog-card No. 72-600281

For sale by the Superintendent of Documents, U.S. Government Printing Office Washington, D.C. 20402 - Price 60 cents (paper cover)

Stock Number 2401-2171 


\section{CONTENTS}

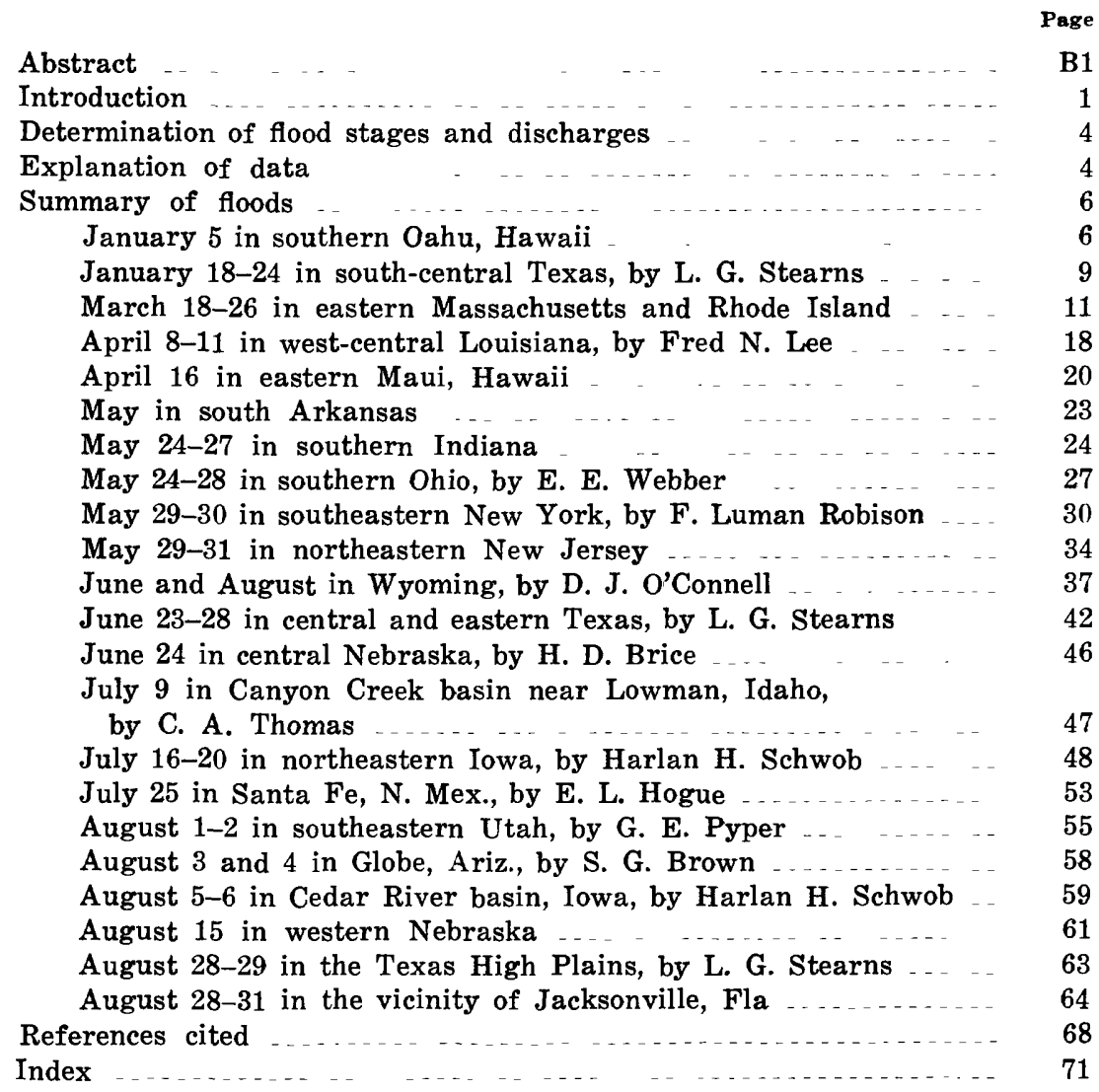

\section{ILLUSTRATIONS}

Figure

1. Map showing areas and months of flood occurrence

in United States

Page

2-17. Maps of flood areas:

2. January 5 in southern Oahu, Hawaii ... 6

3. January 18-24 in south-central Texas . . - 10 
Figures 2-17. Maps of flood areas-Continued

4. March 18-26 in eastern Massachusetts and Rhode Island

5. April 8-11 in west-central Louisiana ... 19

6. April 16 in eastern Maui, Hawaii _..... 21

7. May in south Arkansas ........... 24

8. May 24-27 in southern Indiana ........ 25

9. May 24-28 in southern Ohio ......... . $\quad 28$

10. May 29-30 in southeastern New York ... 31

11. May 29-31 in northeastern New Jersey - $\quad 35$

12. June and August in north-central Wyoming

13. June and August in southwestern Wyoming _ . . .... . . . ....... 40

14. June $23-28$ in central and eastern Texas 43

15. June 24 in central Nebraska ... . . . 46

16. July 9 in Canyon Creek basin near Lowman, Idaho

17. July 16-20 in northeastern Iowa ....... 50

18. Graph of accumulated rainfall, July $16-17$ at

three stations in northeastern Iowa .... .... . 51

19-25. Maps of flood areas:

19. July 25 in Santa Fe, N. Mex .... 54

20. August 1-2 in southeastern Utah _.... 56

21. August 3 and 4 in Globe, Ariz ....... 58

22. August 5-6 in Cedar River basin, Iowa - 60

23. August 15 in western Nebraska ....... 62

24. August $28-29$ in the Texas High Plains -. 63

25. August $28-31$ in the vicinity of Jacksonville, Fla

26. Graph of relation of August 28-31 flood peaks to major flood peaks of record in the vicinity of Jacksonville, Fla

\section{TABLES}

TABLES 1-11. Flood stages and discharges:

1. January 5 in southern Oahu, Hawaii

2. January 18-24 in south-central Texas

3. March $18-26$ in eastern Massachusetts and Rhode Island

4. April 8-11 in west-central Louisiana

5. April 16 in eastern Maui, Hawaii ....... 22

6. May 24-27 in southern Indiana ........ 25

7. May 24-28 in southern Ohio . . . . . . . . 29

8. May 29-30 in southeastern New York ... 32 
TABLES 1-11. Flood stages and discharges-Continued

9. May 29-31 in northeastern New Jersey B36

10. June and August in Wyoming. . . 41

11. June $23-28$ in central and eastern Texas 44

12. Rainfall, in inches, June 23-25 in central $\mathrm{Ne}$ braska

13-17. Flood stages and discharges:

13. June 24 in central Nebraska

14. July 16-20 in northeastern Iowa ... . 52

15. July 25 in Sante Fe, N. Mex ...

16. August 1-2 in southeastern Utah . . . $\quad 57$

17. August 5-6 in Cedar River basin, Iowa - 60

18. August flood elevations in Dry Run Creek basin,

Cedar Falls, Iowa

19-21. Flood stages and discharges:

19. August 15 in western Nebraska

20. August $28-29$ in the Texas High Plains

21. August $28-31$ in vicinity of Jacksonville, 


\title{
SUMMARY OF FLOODS IN THE UNITED STATES DURING 1968
}

\author{
By J. O. Rostvedt and others
}

\section{ABSTRACT}

This report describes the most outstanding floods in the United States during 1968. The four areas of most destructive flooding occurred on May 29-31 in northeastern New Jersey and southeastern New York, on March 18-26 in eastern Massachusetts and Rhode Island, on May 9-17 in southern Arkansas, and on July 16-20 in northeastern Iowa.

Heavy rains on May 28-30 in northeastern New Jersey and southeastern New York caused major flooding. Many peak discharges, especially in streams in New Jersey, exceeded that of the 50-year flood. Eight lives were lost because of the floods, and the Small Business Administration estimated the flood damage in New Jersey at $\$ 133$ million.

March 1968 was one of the wettest Marches of record in Massachusetts and Rhode Island, where total rainfall ranged from 7 to 12 inches. Most of this rain fell in two periods, March 12-13 and 17-19. This heavy rain caused outstanding floods on March 18-26. Peak discharges in many streams exceeded previous maximums; some were the greatest since 1886 . No lives were lost in the floods, but damage was estimated at $\$ 28$ million in Massachusetts and $\$ 9$ million in Rhode Island.

Heavy rain fell in southern Arkansas on May 7-18 over an area extending from Little Rock to the southwest corner of the State. The wetting of the soil from a storm of May 9-10 was conducive to flooding following a second storm on May 13. Flooding was severe and widespread, millions of acres of land were inundated, and damage amounted to $\$ 18.3$ million.

A severe storm occurred in the central part of northeastern Iowa on the night of July 16-17. The storm caused record floods in the central part of the Wapsipinicon River basin and in tributaries of the Cedar River. Four lives were lost. A flood-control dam on Virden Creek was overtopped and the outlet structure was washed out. Damage was estimated at $\$ 14.5$ million.

In addition to the four areas of flooding mentioned above, 20 others of lesser magnitude are considered important enough to be included in this annual flood summary.

\section{INTRODUCTION}

This report summarizes information on outstanding floods in the United States during 1968. The floods reported were unusual 
hydrologic events in which large areas were affected, great damage resulted, or record-high discharges or stages occurred and in which sufficient data were available for the preparation of a report.

Water-Supply Paper 1970-A, "Floods of May 1968 in South Arkansas" (Gilstrap, 1972) is a special report which describes those floods in detail. The areas for which flood reports have been prepared for 1968 are shown in figure 1. The area discussed in chapter $\mathrm{A}$ is indicated by a dot pattern, and other areas discussed in this summary chapter are shown by a line pattern. The months in which the floods occurred are shown.

A flood may be defined as any abnormally high streamflow that overtops natural or artificial banks of a stream; every year, a great number of these events occur that are unreported.

Each flood in this report was selected as an outstanding or relatively rare event. A rare flood is not necessarily an impressive flood, but it is one whose probability of being duplicated at any one site is small. A rare flood in an isolated area or in a sparsely inhabited area could possibly be a more outstanding hydrologic event than a much publicized flood in a developed area.

Innumerable combinations of the variable factors of meteorology and physiography produce floods of all degrees and severity. Some meteorological factors influencing floods are the form, amount, duration, and intensity of precipitation; amount of previous precipitation, which would affect the moisture absorption of the soil; the temperature, which may cause frozen soil or may determine the rate of snowmelt; and the direction of storm movement. The principal physiographic features of a basin that determine flood flows are drainage area, altitude, geology, shape, slope, aspect, and vegetative cover. With the exception of vegetative cover and soil preconditioning the physiographic features are fixed for any given area. The combination of the magnitude and intensity of meteorologic phenomena, the antecedent moisture conditions, and the effect of inherent physiographic features on runoff determines what the magnitude of a flood will be.

According to U.S. Weather Bureau data, losses from floods in the United States during 1968 (\$339 million) were about 85 percent of the national annual average of $\$ 400$ million, based on the 15-year period 1951-65 and adjusted to the 1965 price index.

Nationally, the number of lives lost because of floods in 1968 was 31 compared with 34 in 1967 and 50 in 1966 and was much less than the annual average of 77 lives lost during the 44-year period, 1925-68. 


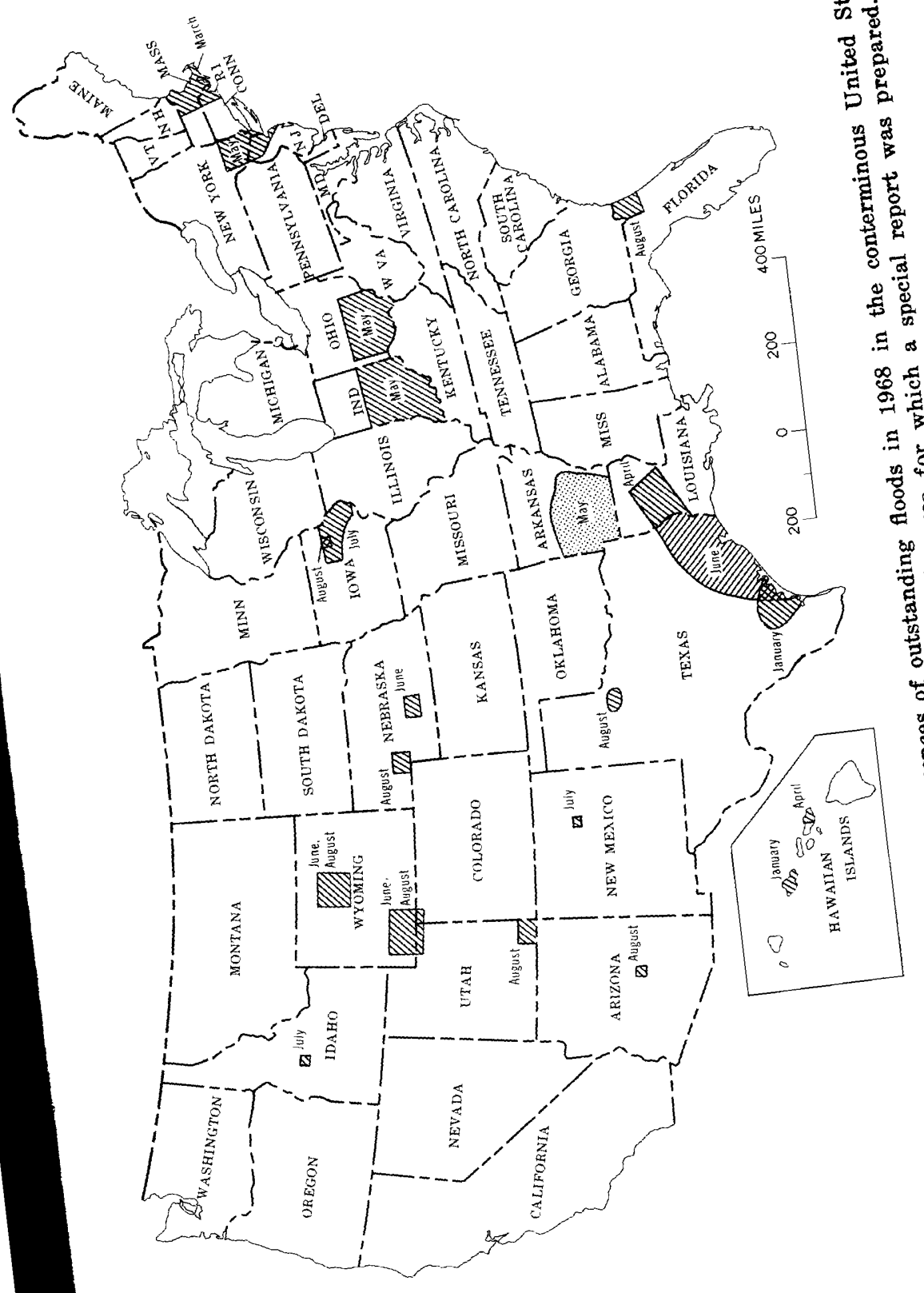


Many of the flood reports give the amount of rainfall and the duration of the storm associated with the flooding. Recurrence intervals of these storms may be determined from the U.S. Weather Bureau (1961) or from a simplified set of isopluvial maps and charts contained in a report by Rostvedt (1965).

Continuing investigation of surface-water resources in the areas covered by this report is performed by the U.S. Geological Survey in cooperation with State agencies, the U.S. Army Corps of Engineers, the Bureau of Reclamation, and other Federal or local agencies. Some data were obtained from U.S. Weather Bureau publications.

Data were collected, computations were made, and most of the text was written by the district offices in whose districts the floods occurred.

\section{DETERMINATION OF FLOOD STAGES AND DISCHARGES}

The usual method of determining stream discharges at a gaging station is the application of a stage-discharge relation to a known stage. The relation at a station is usually defined by current-meter measurements through as much of the range of stage as possible. If the peak discharge at a station is above the range of the computed stage-discharge relation, short extensions may be made to the graph of relation by logarithmic extrapolation, by velocity-area studies, or by the use of other measurable hydraulic factors.

Peak discharges that are greatly above the range of the stage-discharge relation at gaging stations and peak discharges at miscellaneous sites that have no developed stage-discharge relation are generally determined by various types of indirect measurements. Adverse conditions often make it impossible to obtain current-meter measurements at some sites during major floods. Peak discharges at these sites are measured, after the floods have subsided, by indirect methods based on detailed surveys of selected channel reaches. A general description of the indirect methods used by the Geological Survey is given by Corbett and others (1943), Johnson (1936), and Dalrymple and others (1937). More detailed information concerning the latest techniques is available in recent reports by Kindsvater and others (1953), Bodhaine (1963), and Tracy (1957).

\section{EXPLANATION OF DATA}

The floods are described in chronological order. Because the 
type and the amount of information differ for the floods, no consistent form can be used to report the events.

The date for each flood include: A description of the storm, the flood, and the flood damage; a map of the flood area showing flood-determination points and, for some storms, precipitation stations or isohyets; rainfall amounts and intensities; and peak stages and discharges of the streams affected.

When considerable rainfall data are available, they are presented in tabular form and show daily or storm totals. When sufficient date are available to determine the pattern and distribution of rainfall, an isohyetal map may be shown.

A summary table of peak stages and discharge is given for each flood unless the number of stations in the report is small, and then the information is included in the text description.

In the summary table the first column under maximum floods gives the period of known floods prior to the 1968 floods. This period does not necessarily correspond to that of gaging station operation, but the period may extend back to an earlier date. More than one period of known floods are shown for some stations. A period is shown whenever it can be associated with a maximum stage, even though the corresponding discharge may not be known. A second, shorter period of floods is then given in which maximums of both discharge and stage are known.

The second column under maximum floods shows the year, within the period of known floods, prior to the 1968 floods being reported, in which maximum stage or discharge occurred. The third column gives the date of the peak stage or discharge of the 1968 floods.

The last column gives the recurrence interval for the 1968 peak discharge. The recurrence interval is the average interval, in years, in which a flood of a given magnitude (the 1968 peak) will be exceeded once as an annual maximum. A flood having a recurrence interval of 20 years is one that can be expected to occur, on the average, once in 20 years or that has a 5-percent chance of occurring in any year. The recurrence intervals in the tables were obtained from U.S. Geological Survey reports on flood magnitude and frequency. In nearly all flood-frequency reports used, the data limit the determination of recurrence intervals to 50 years; in a few reports the limit is less than 50 years. The severity of a flood whose recurrence interval exceeds the limit of determination is expressed as the ratio of its peak discharge to the discharge of the flood that has a recurrence interval equal to the limits of determination. 


\section{SUMMARY OF FLOODS}

\section{FLOODS OF JANUARY 5 IN SOUTHERN OAHU, HAWAII ${ }^{x}$}

Development of deep and nearly stationary low-pressure areas at upper levels and at the surface caused cold and unstable weather conditions over the Hawaiian Islands.

Rainfall on Oahu during the night of January 4 was heavy on the leeward slopes of the Koolau Range extending from Wahiawa to the Makiki Valley area. The heaviest rain, beginning about 9:30 p.m. on the 4th and extending through the early morning hours of the 5th, fell over the central highlands of the island (fig. 2). The high intensity rainfall caused severe flooding and extensive damages to areas in Waiawa, Pearl City, and Waimalu.

The Pearl City Terrace station in Pearl City recorded 6.77

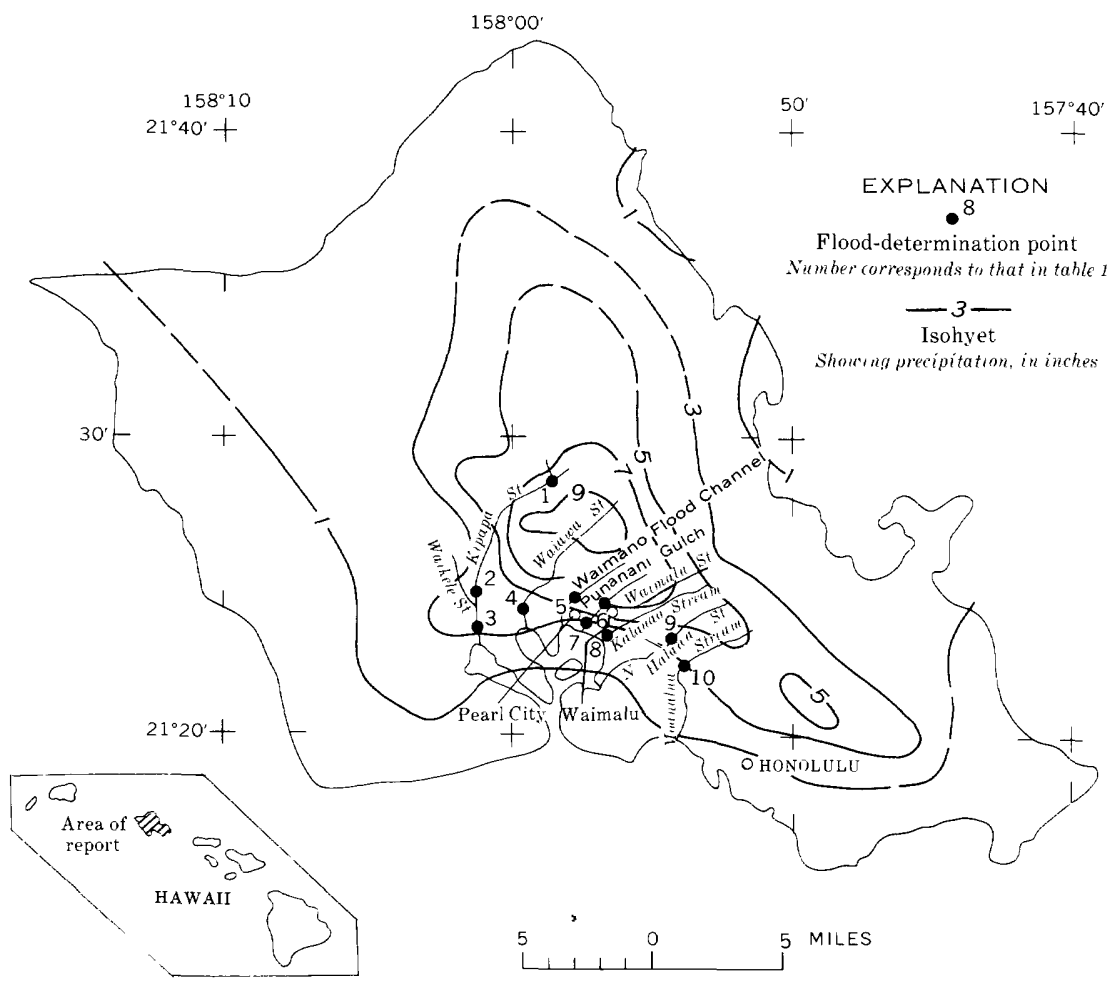

FIGURE 2.-Flood area; location of flood-determination points and isohyets for January 5 , floods of January 5 in southern Oahu, Hawaii.

\footnotetext{
${ }^{1}$ Modified from U.S. Corns of Engineers (1968a).
} 
inches of rain from 9:00 p.m. on the 4th through 5:00 a.m. on the 5 th ; 2.78 inches fell during the first $21 / 2$ hours, and after stopping for almost 3 hours, a heavy burst dumped 3.97 inches in the last 2 hours. Of this total, slightly more than 2 inches fell between $3: 15$ a.m. and $3: 30$ a.m.; also 3.57 inches were recorded between $3: 15$ a.m. and 4:00 a.m. Intensities in the area have recurrence intervals of over 100 years.

Two unofficial plantation gages on higher ground above Pearl City reported 9.08 and 9.40 inches of rain for the 24-hour period ending at 8:00 a.m. on January 5. Most of the rain fell during the night and the early morning hours. Stations located in other parts of the island recorded scattered amounts of rainfall ranging from 0 to 3.31 inches. None of these stations reported any unusual amounts or intensities.

Waiawa Stream, with 16 years of record, had a discharge of $23,400 \mathrm{cfs}$ (cubic feet per second). The Waimano Flood Channel had a discharge of $3,400 \mathrm{cfs}$ at a site 0.5 mile upstream from the gage. Additional runoff between the measurement site and the gage did not enter the channel. Punanani Gulch, a miscellaneous measurement site tributary to Waimalu Stream, had a discharge of $4,500 \mathrm{cfs}$. Slope-area measurements were completed by U.S. Geological Survey personnel to determine peak discharges during this storm. A summary of streamflow data is presented in table 1.

TABLE 1.-Flood stages and discharges, January 5 in southern Oahu, Hawaii

\begin{tabular}{|c|c|c|c|c|c|c|c|}
\hline \multirow{3}{*}{ No. } & \multirow{3}{*}{$\begin{array}{l}\text { Stream and place } \\
\text { of determination }\end{array}$} & \multirow{3}{*}{$\begin{array}{l}\text { Drainage } \\
\text { area } \\
\text { (sq mi) }\end{array}$} & \multicolumn{5}{|c|}{ Maximum floods } \\
\hline & & & \multicolumn{2}{|c|}{$\begin{array}{l}\text { Known before } \\
\text { January } 1968\end{array}$} & \multirow{2}{*}{$\begin{array}{l}\text { January } \\
1968\end{array}$} & \multirow{2}{*}{$\begin{array}{c}\text { Gage } \\
\text { height } \\
\text { (feet) }\end{array}$} & \multirow{2}{*}{$\begin{array}{l}\text { Discharge } \\
\text { (cfs) }\end{array}$} \\
\hline & & & Period & Year & & & \\
\hline 1 & $\begin{array}{l}\text { Kipapa Stream near } \\
\text { Wahiawa. }\end{array}$ & 4.29 & $1957-68$ & 1963 & 5 & $\begin{array}{r}12.29 \\
8.12\end{array}$ & $\begin{array}{l}\mathbf{5}, 680 \\
1,520\end{array}$ \\
\hline 2 & Kipapa Stream near & 13.8 & $1967-68$ & 1967 & o & $\begin{array}{l}8.12 \\
9.08\end{array}$ & 1,090 \\
\hline 3 & $\begin{array}{l}\text { Waipahu. } \\
\text { Waikele Stream at }\end{array}$ & 45.7 & $1951-68$ & 1954 & 5 & $\begin{array}{l}16.5 \\
114.82\end{array}$ & $\begin{array}{r}7,300 \\
13,600\end{array}$ \\
\hline & Waipahu. & & & & 5 & 9.72 & 7,360 \\
\hline 4 & $\begin{array}{l}\text { Waiawa Stream near } \\
\text { Pearl City. }\end{array}$ & 26.4 & $\begin{array}{l}1952-68 \\
\ldots\end{array}$ & 1954 & 5 & $\begin{array}{l}19.27 \\
20.56\end{array}$ & $\begin{array}{l}16,900 \\
23,400\end{array}$ \\
\hline 5 & $\begin{array}{l}\text { Waimano Flood } \\
\text { Channel at } \\
\text { Pearl City. }\end{array}$ & ... & $\ldots$ & $\ldots$ & 5 & $\ldots$ & 3,400 \\
\hline 6 & $\begin{array}{l}\text { Punanani Gulch at } \\
\text { Waiau. }\end{array}$ & $\ldots$ & - & . & 5 & $\cdots$ & 5,400 \\
\hline 7 & $\begin{array}{l}\text { Waimalu Stream } \\
\text { at Aiea. }\end{array}$ & 6.07 & $\begin{array}{l}1952-62 \\
1963-68 .\end{array}$ & 1965 & $\cdots$ & 6.30 & 6,930 \\
\hline 8 & $\begin{array}{l}\text { Kalauao Stream at } \\
\text { Moanalua Road } \\
\text { at Aiea. }\end{array}$ & 2.59 & $1957-68$ & 1963 & 5 & $\begin{array}{l}6.82 \\
6.63 \\
3.56\end{array}$ & $\begin{array}{l}8,100 \\
2,580 \\
1,200\end{array}$ \\
\hline 9 & $\begin{array}{l}\text { North Halawa } \\
\text { Stream near }\end{array}$ & 3.45 & $\begin{array}{l}1939-33, \\
1953-68 .\end{array}$ & 1932 & $\cdots$ & 13.36 & 6,650 \\
\hline 10 & $\begin{array}{l}\text { Aiea. } \\
\text { Moanaiua Stream } \\
\text { near Honolulu. }\end{array}$ & 2.73 & $1926-68$ & $\begin{array}{l}1930 \\
-\ldots\end{array}$ & $\begin{array}{r}5 \\
5\end{array}$ & $\begin{array}{r}10.40 \\
11.58 \\
7.63\end{array}$ & $\begin{array}{l}1,210 \\
4,580 \\
1,160\end{array}$ \\
\hline
\end{tabular}

${ }^{1}$ At site 300 feet downstream at datum 1.30 feet higher. 
Extremely high flow in Punanani Gulch, a tributary to Waimalu Stream, caused extensive damage to Waimalu Elementary School. The normally dry gulch, without a defined channel in the lower reaches, carried the rampaging waters through the sugarcane fields, across the highway, and slammed huge tree trunks and other debris against the school building. Water up to $41 / 2$ feet deep smashed through six classrooms, washing furniture, books, supplies, and other equipment into the river. Three automobiles and a converted garage storing school furniture and appliances were completely demolished. Debris and mud up to 2 feet deep covered the school grounds. Damage to the school was about $\$ 75,000$. Damage to homes in the flood area was minimal owing to the high floor lines. Equipment and other property in a contractor's yard was also damaged by the floodwater. Total damage to private property amounted to about $\$ 25,000$.

Backwater created by blocked culverts in the Waimano Flood Channel caused considerable damage to public and private properties in the Pearl City area during the early morning flood. The Pearl City Shopping Center was inundated to a depth of 6 feet. Ten business establishments lost an estimated $\$ 745,000$ in damage.

Two bridges consisting of double box culverts slightly upstream from the shopping center were clogged with debris. Chain link fences were washed into the stream forming efficient barriers and nets in the culverts, stopping debris as it floated by. As a result, the floodwaters rose more than 8 feet above the 10foot-deep channel. More than 20 homes were inundated to 1-foot depth and several others, to a depth of 4-5 feet. Five homes were severely damaged when swept off their foundations. Plants, small trees, sheds, and a car were swept downstream. Water overflowed at an upper culvert sending a stream 3-4 feet deep rushing down a two-block long street and washing everything away within its path. Three other cars were destroyed, and two large trailer dump trucks were swept more than 100 feet down the street. One home near the culvert was lifted from the foundation and turned around by the force of the water. A private childeren's day care center lost $\$ 5,000$ worth of school equipment, furniture, and supplies.

Farther upstream, another blocked culvert sent water over the road and through two more homes, which caused a considerable amount of damage. The estimated damages to residential property in the Pearl City area were about $\$ 140,000$.

Water over the right bank of the channel upstream from the 
Noelani Street culvert undermined and destroyed 450 feet of the rubble masonry wall. Damages to the Waimano Flood Channel totaled about $\$ 158,000$. A concrete-lined channel at the Pacific Palisades Drainageway in the newly developed Pacific Palisades subdivision was damaged during the storm.

Damage in the lowland areas of Waiawa was extensive. Seven homes near the Ewa-Schofield Junction at Kamehameha Highway were totally destroyed, and 12 others were severely damaged by high water. The river rose more than 15 feet above the banks, carried the homes more than 400 yards downstream, and crushed them together near the highway bridge. Banana fields were leveled, many head of livestock were drowned, and huge quantities of household goods and furniture were lost.

Areas downstream from the Kamehameha Highway bridge were flooded. The rampaging stream went over the low banks again and damaged a home and an automobile. A sewage treatment plant was also damaged. The low-lying area, nearly at sea level, is sparsely populated and used mainly for agriculture. Nine acres of crops were destroyed and a number of cattle, hogs, and poultry were lost.

Damages to residences in Waiawa totaled about $\$ 69,000$; and agricultural losses, $\$ 33,600$.

FLOODS OF JANUARY 18-24 IN SOUTH-CENTRAL TEXAS

By L. G. STEARNS

Frequent rains, accompanied by an occasional intense rainstorm, made the month of January one of the wettest Januarys on record at many weather stations in south-central Texas.

The U.S. Weather Bureau at San Antonio recorded a monthly rainfall of 8.52 inches, the greatest amount ever recorded in January at that station since records began in 1871. Above-normal rainfall occurred during the month at nearly all stations in the area. During the period January 18-21, heavy rains of 10 or more inches caused flooding from San Antonio southward to the Gulf Coast (fig. 3). During the period January 18-24, the basins of the Guadalupe and Nueces Rivers and their tributaries were the principal basins affected by flooding (table 2). Several adjacent smaller basins were affected to a lesser extent. Although record rainfall totals were established during January at several stations, floods were not the maximum of record at any gaging station during this period.

Flash flooding in San Antonio caused five deaths and property damage estimated at $\$ 4$ million. Bexar County was declared a disaster area for the first time in history. 


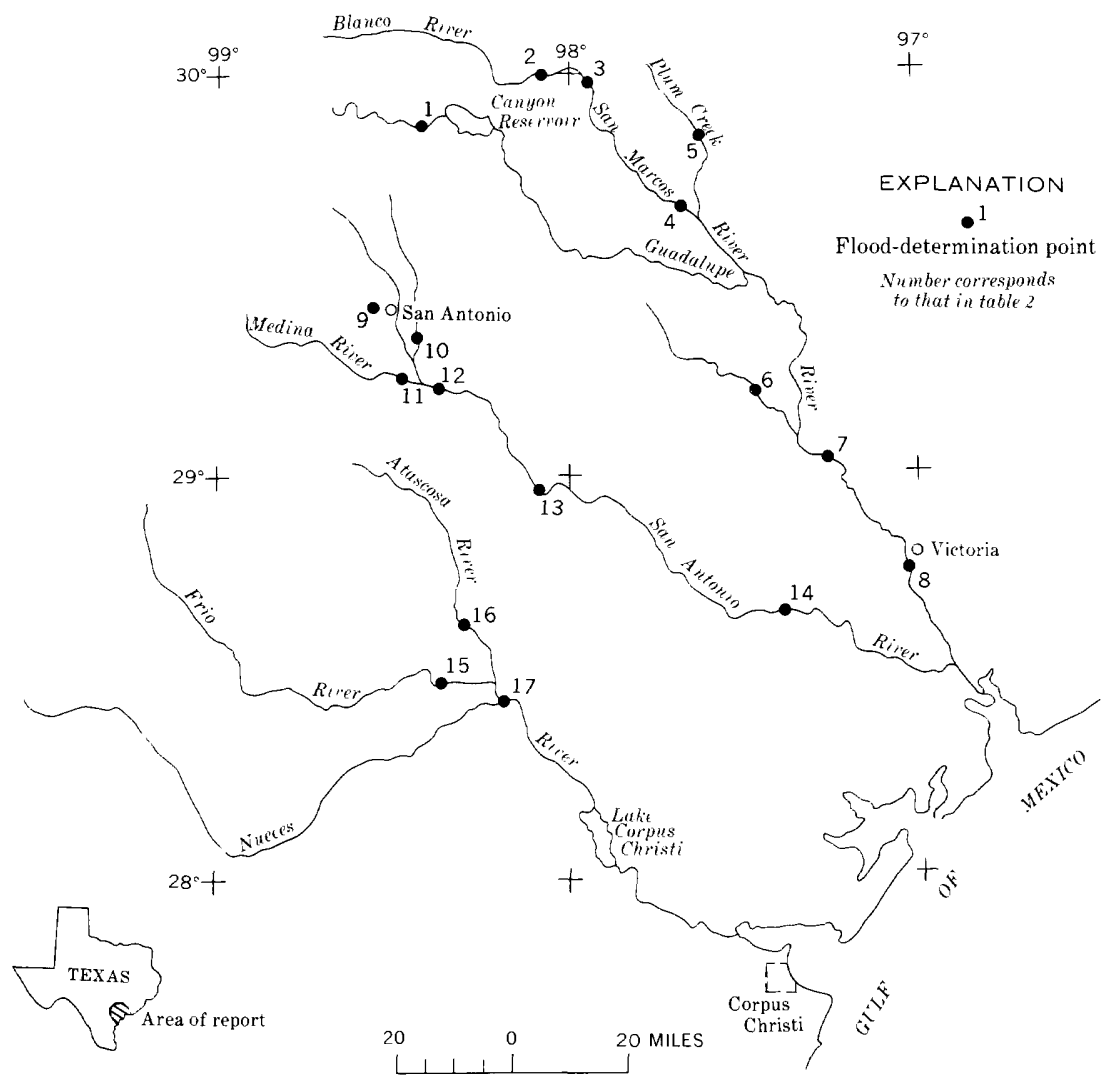

Figure 3.-Flood area; location of flood-determination points, floods of January $18-24$ in south-central Texas.

TABLE 2.-Flood stages and discharges, January 18-24 in south-central Texas

\begin{tabular}{|c|c|c|c|c|c|c|c|c|}
\hline \multirow{4}{*}{ No. } & \multirow{4}{*}{$\begin{array}{l}\text { Stream and place } \\
\text { of determinaiton }\end{array}$} & \multirow{4}{*}{$\begin{array}{l}\text { Drainage } \\
\text { area } \\
(\mathrm{sq} \mathrm{mi})\end{array}$} & \multicolumn{6}{|c|}{ Maximum floods } \\
\hline & & & \multirow{2}{*}{\multicolumn{2}{|c|}{$\begin{array}{l}\text { Known before } \\
\text { January } 1968\end{array}$}} & \multirow{3}{*}{$\underset{1968}{\text { January }}$} & \multirow{3}{*}{$\begin{array}{c}\text { Gage } \\
\text { height } \\
\text { (feet) }\end{array}$} & \multicolumn{2}{|c|}{ Discharge } \\
\hline & & & & & & & & Recur- \\
\hline & & & Period & Year & & & Cfs & $\begin{array}{c}\text { interval } \\
\text { (years) }\end{array}$ \\
\hline \multicolumn{9}{|c|}{ Guadalupe River basin } \\
\hline 1 & $\begin{array}{l}\text { Guadalupe River } \\
\text { near Spring }\end{array}$ & 1,282 & $\begin{array}{l}1859-1968 \\
1922-68\end{array}$ & $\begin{array}{l}1869 \\
1932\end{array}$ & $\overline{20}$ & $\begin{array}{l}53 \\
42.10\end{array}$ & $\begin{array}{c}\left({ }^{1}\right) \\
121,000\end{array}$ & $-\overline{2}$ \\
\hline 2 & $\begin{array}{l}\text { Blanco River at } \\
\text { Wimberley. }\end{array}$ & 364 & $1869-1968$ & 1929 & 20 & $\begin{array}{l}31.10 \\
12.90\end{array}$ & $\begin{array}{r}113,000 \\
18,200\end{array}$ & 4 \\
\hline 3 & $\begin{array}{l}\text { Blanco River } \\
\text { near Kyle. }\end{array}$ & 424 & $1882-1968$ & 1929 & 20 & $\begin{array}{l}40 \\
23.33\end{array}$ & $\begin{array}{r}139,000 \\
26,600\end{array}$ & 5 \\
\hline 4 & $\begin{array}{l}\text { San Marcos River } \\
\text { at Luling. }\end{array}$ & 833 & $\begin{array}{l}1859-1968 \\
1939-68\end{array}$ & $\begin{array}{l}1869 \\
1952\end{array}$ & $\overline{2} \overline{1}$ & $\begin{array}{l}40.4 \\
34.95 \\
31.42\end{array}$ & $\begin{array}{c}(1) \\
57,000 \\
24,800\end{array}$ & $\begin{array}{ll}- \\
-- \\
3\end{array}$ \\
\hline 5 & $\begin{array}{l}\text { Plum Creek at } \\
\text { Lockhart. }\end{array}$ & 113 & $\begin{array}{r}1905-68 \\
1959-68\end{array}$ & $\begin{array}{l}1936 \\
1960\end{array}$ & $\begin{array}{l}21 \\
\overline{21}\end{array}$ & $\begin{array}{l}222 \\
20.62 \\
15.92\end{array}$ & $\begin{array}{r}\left({ }^{1}\right) \\
26,600 \\
4,350\end{array}$ & - \\
\hline
\end{tabular}

See footnotes at end of table. 
TABLE 2.-Flood stages and discharges, January 18-24 in south-central Texas-Continued

\begin{tabular}{|c|c|c|c|c|c|c|c|c|}
\hline \multirow{4}{*}{ No. } & \multirow{4}{*}{$\begin{array}{l}\text { Stream and place } \\
\text { of determination }\end{array}$} & \multirow{4}{*}{$\begin{array}{l}\text { Drainage } \\
\text { area } \\
(\mathrm{sq} \mathrm{mi})\end{array}$} & \multicolumn{6}{|c|}{ Maximum floods } \\
\hline & & & \multirow{2}{*}{\multicolumn{2}{|c|}{$\begin{array}{l}\text { Known before } \\
\text { January } 1968\end{array}$}} & \multirow{3}{*}{$\underset{1968}{\text { January }}$} & \multirow{3}{*}{$\begin{array}{l}\text { Gage } \\
\text { height } \\
\text { (feet) }\end{array}$} & \multicolumn{2}{|c|}{ Discharge } \\
\hline & & & & & & & \multirow[b]{2}{*}{ Cfs } & \multirow{2}{*}{$\begin{array}{l}\text { Recur- } \\
\text { rence } \\
\text { interval } \\
\text { (years) }\end{array}$} \\
\hline & & & Period & Year & & & & \\
\hline \multicolumn{9}{|c|}{ Guadalupe River basin-Continued } \\
\hline 6 & \multirow{3}{*}{$\begin{array}{l}\text { Sandies Creek } \\
\text { near Westhoff. } \\
\text { Guadalupe River } \\
\text { at Cuero. }\end{array}$} & 560 & 1864-1968 & 1936 & $2 \overline{2}$ & $\begin{array}{l}33.1 \\
22.82\end{array}$ & \multirow{2}{*}{$\begin{array}{r}92,700 \\
6,550 \\
\left({ }^{1}\right) \\
101,000\end{array}$} & $<2$ \\
\hline 7 & & 4,877 & $\begin{array}{l}1900-68 \\
1903-06 \\
1917-68\end{array}$ & $\begin{array}{l}1936 \\
1929\end{array}$ & $\cdots$ & $\begin{array}{l}44.33 \\
3 \mathbf{3 5 . 2}\end{array}$ & & - \\
\hline & & & - - - - & $\cdots$ & 23 & 33.81 & \multirow{3}{*}{$\begin{array}{r}44,200 \\
179,000 \\
179,000 \\
44,300 \\
15,300 \\
5,730\end{array}$} & $\begin{array}{r}6 \\
--\end{array}$ \\
\hline 8 & \multirow{3}{*}{$\begin{array}{l}\text { Guadalupe River } \\
\text { at Victoria. } \\
\text { San Antonio } \\
\text { River at } \\
\text { San Antonio. } \\
\text { Salado Creek } \\
\text { (lower station) } \\
\text { at San Antonio. }\end{array}$} & 5,161 & $1833-1968$ & 1936 & $2 \overline{5}$ & \multirow{2}{*}{$\begin{array}{r}31.22 \\
29.72 \\
320.14 \\
11.23\end{array}$} & & 6 \\
\hline 9 & & 42 & $1819-1968$ & $\overline{19} \overline{2} \overline{1}$ & $1 \ddot{8}$ & & & 8 \\
\hline 10 & & 189 & $\begin{array}{l}1941-68 \\
1961-68\end{array}$ & $\begin{array}{l}1946 \\
1960 \\
1965\end{array}$ & & \multirow{2}{*}{$\begin{array}{l}26.8 \\
26.8 \\
22.11 \\
22.88 \\
39.23 \\
28.56\end{array}$} & \multirow{2}{*}{$\begin{array}{c}\left({ }^{1}\right) \\
\left({ }^{1}\right) \\
6,090 \\
6,630 \\
31,800 \\
13,100\end{array}$} & 3 \\
\hline 11 & $\begin{array}{l}\text { Medina River } \\
\text { near San } \\
\text { Antonio. }\end{array}$ & 1,317 & $1912-68$ & $\overline{19} \overline{4} \overline{6}$ & $\begin{array}{l}18 \\
18\end{array}$ & & & $<2$ \\
\hline 12 & $\begin{array}{l}\text { San Antonio River } \\
\text { near Elmendorf. }\end{array}$ & 1,743 & $\begin{array}{l}1900-1968 \\
1963-68 \\
\end{array}$ & $\begin{array}{l}1946 \\
1965\end{array}$ & $\overline{18}$ & $\begin{array}{l}61 \\
35.34 \\
40.15\end{array}$ & $\begin{array}{c}\left({ }^{(1)}\right) \\
15,100 \\
29,800\end{array}$ & $\overline{25}$ \\
\hline 13 & \multirow{2}{*}{$\begin{array}{l}\text { San Antonio River } \\
\text { near Falls City. } \\
\text { San Antonio River } \\
\text { at Goliad. }\end{array}$} & 2,113 & $1875-1968$ & $\overline{19} 46$ & 21 & $\begin{array}{l}33.80 \\
22.87\end{array}$ & $\begin{array}{l}47,400 \\
17,300\end{array}$ & 8 \\
\hline 14 & & 3,921 & $\begin{array}{c}1869-1968 \\
-\end{array}$ & $\begin{array}{r}1967 \\
-\cdots \\
\end{array}$ & $\overline{24}$ & $\begin{array}{l}53.7 \\
41.98\end{array}$ & $\begin{array}{r}138,000 \\
25,900 \\
\end{array}$ & 8 \\
\hline \multicolumn{9}{|c|}{ Nueces River basin } \\
\hline 15 & \multirow{3}{*}{$\begin{array}{l}\text { Frio River at } \\
\text { Calliham. } \\
\text { Atascosa River } \\
\text { at Whitesett. } \\
\text { Nueces River near } \\
\text { Three Rivers. }\end{array}$} & 5,491 & 1870-1968 & 1932 & $\overline{2} \overline{1}$ & \multirow{3}{*}{$\begin{array}{l}39.2 \\
29.84 \\
41.3 \\
35.93 \\
49.21 \\
40.64\end{array}$} & \multirow{3}{*}{$\begin{array}{r}109,000 \\
12,700 \\
121,000 \\
23,500 \\
141,000 \\
36,000\end{array}$} & 3 \\
\hline 16 & & 1,171 & $1881-1968$ & 1967 & $\overline{2} \bar{I}$ & & & is \\
\hline 17 & & 15,600 & $1875-1968$ & 1967 & $\overline{2} \overline{2}$ & & & 6 \\
\hline
\end{tabular}

1 Not determined.

2 At site 548 feet upstream.

"At former site and datum.

\section{FLOODS OF MARCH 18-26 IN EASTERN MASSACHUSETTS AND RHODE ISLAND :}

Precipitation during March in eastern Massachusetts and Rhode Island was considerably above normal. The U.S. Weather Bureau reported that, in much of the area, March 1968 was one of the wettest Marches of record with monthly totals as high as 7 to 12 inches. Most of the precipitation fell during two stormsthe first on March 12-13, the second on March 17-19. Precipitation during the first storm was generally much less than that during the second, but with runoff from snow still remaining in sheltered areas, the earlier storm contributed to the severe flooding. In addition, the time of the year-prior to the start of the growing season-increased the magnitude of the flood peaks and volume of runoff.

\footnotetext{
${ }^{2}$ Modified from G. K. Wood, L. A. Swallow, C. G. Johnson, and G. H. Searles (1970).
} 
The Weather Bureau reported that at many stations new 24hour precipitation records were established for March. Blue Hill Observatory at Milton, Mass., reported a 24-hour total of 6.62 inches on March 17 and 18. An isohyetal map for Massachusetts and Rhode Island, adapted from a map prepared by the Weather Bureau, is presented in figure 4 .

The flooding of March 18-26 was outstanding. Many streams with long-term streamflow records had flood peaks which exceeded previous maximums (table 3). For Ipswich River near Ipswich, Mass., (No. 19, table 3) the March peak flow exceeded

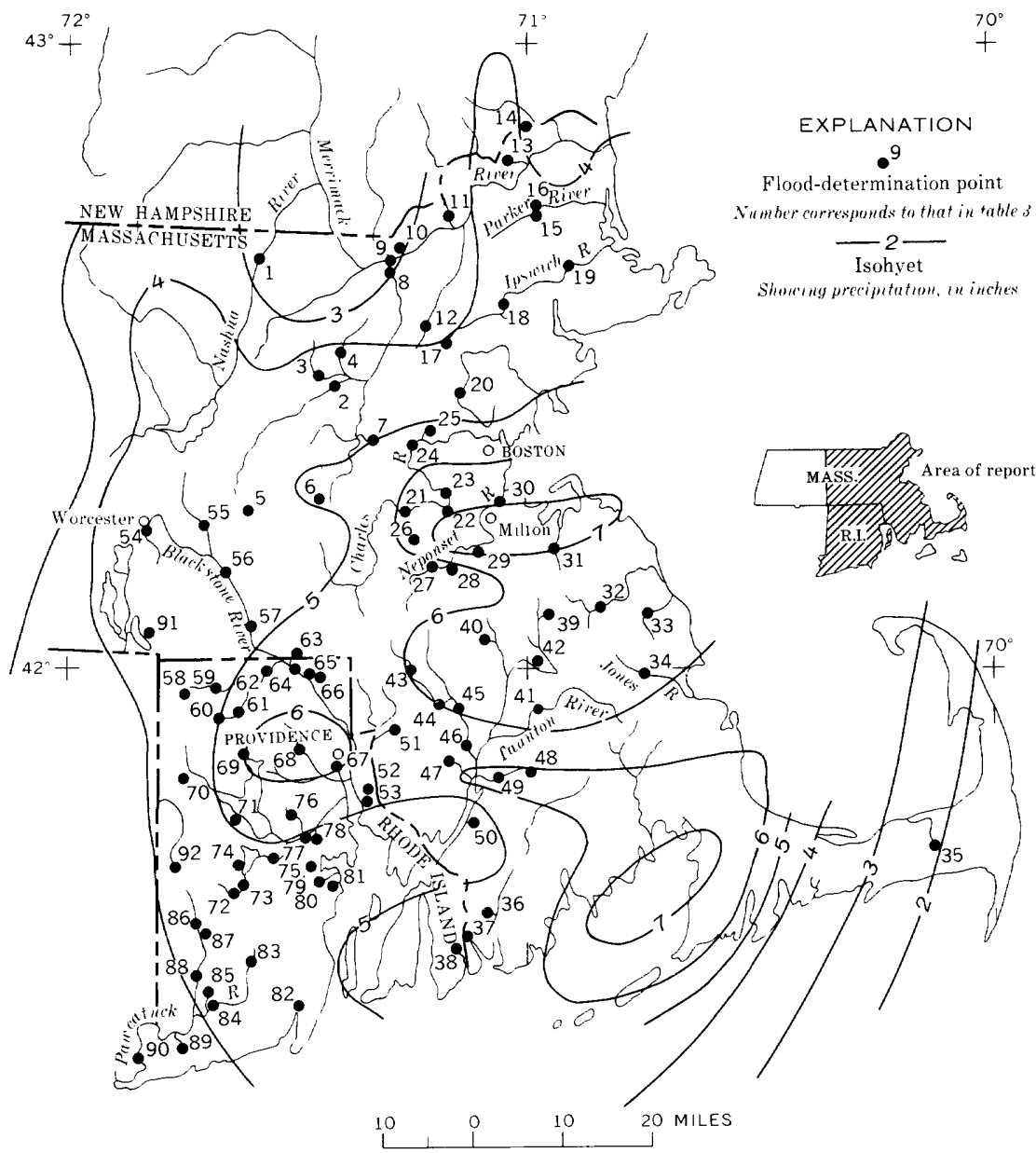

FIgURE 4.-Flood area; location of flood-determination points and isohyets for March 17-19, floods of March 18-26 in eastern Massachusetts and Rhode Island. 
TABLE 3.-Flood stages and discharges, March 18-26 in eastern Massachusetts and Rhode Island

\begin{tabular}{|c|c|c|c|c|c|c|c|c|}
\hline \multirow{4}{*}{ No. } & \multirow{4}{*}{$\begin{array}{l}\text { Stream and place } \\
\text { of determination }\end{array}$} & \multirow{4}{*}{$\begin{array}{l}\text { Drainage } \\
\text { area } \\
\text { (sq mi) }\end{array}$} & \multicolumn{6}{|c|}{ Maximum floods } \\
\hline & & & \multirow{2}{*}{\multicolumn{2}{|c|}{$\begin{array}{l}\text { Known before } \\
\text { March } 1968\end{array}$}} & \multirow{3}{*}{$\underset{1968}{\text { March }}$} & \multirow{3}{*}{$\begin{array}{l}\text { Gage } \\
\text { height } \\
\text { (feet) }\end{array}$} & \multicolumn{2}{|c|}{ Discharge } \\
\hline & & & & & & & & Recur- \\
\hline & & & Period & Year & & & Cfs & $\begin{array}{c}\text { interval } \\
\text { (years) }\end{array}$ \\
\hline \multicolumn{9}{|c|}{ Merrimack River basin } \\
\hline 1 & $\begin{array}{l}\text { Nashua River at } \\
\text { East Pepperell, } \\
\text { Mass. }\end{array}$ & ${ }^{1} 316$ & $1936-68$ & 1936 & 20 & $\begin{array}{l}19.1 \\
11.7\end{array}$ & $\begin{array}{r}20,900 \\
6,900\end{array}$ & ()$\left.^{3}\right)$ \\
\hline 2 & $\begin{array}{l}\text { Assabet River at } \\
\text { Maynard, Mass. }\end{array}$ & 116 & $\begin{array}{c}1887-1968 \\
-\end{array}$ & $\begin{array}{l}1955 \\
1955\end{array}$ & & +8.96 & 4,250 & $\ldots$ \\
\hline ; & $\begin{array}{l}\text { Heath Hen } \\
\text { Meadow Brook }\end{array}$ & 3.89 & $1964-68$ & 1967 & 18 & $\begin{array}{l}8.15 \\
5.41 \\
9.80\end{array}$ & $\begin{array}{r}3,620 \\
54 \\
156\end{array}$ & $\begin{array}{l}{ }^{6} 1.1 \\
-\left({ }^{3}\right)\end{array}$ \\
\hline 4 & $\begin{array}{l}\text { Nashaba Brook } \\
\text { near Acton, } \\
\text { Mass. }\end{array}$ & 12.7 & $\begin{array}{r}1964-68 \\
-\ldots\end{array}$ & 1967 & $19-20$ & $\begin{array}{l}4.11 \\
5.07\end{array}$ & $\begin{array}{l}128 \\
360\end{array}$ & $\left({ }^{3}\right)$ \\
\hline 5 & $\begin{array}{l}\text { Mass. } \\
\text { Jackstraw Brook } \\
\text { at Westboro, } \\
\text { Mass. }\end{array}$ & 1.11 & $\begin{array}{r}1964-68 \\
-\end{array}$ & $\begin{array}{l}1964 \\
-\end{array}$ & 18 & $\begin{array}{l}11.9 .7 \\
12.72\end{array}$ & $\begin{array}{l}27 \\
27\end{array}$ & $\left({ }^{3}\right)$ \\
\hline 6 & $\begin{array}{c}\text { Sudbury River at } \\
\text { Framingham } \\
\text { Center, Mass. }\end{array}$ & 75.2 & $1875-1968$ & 1955 & $18^{-}$ & 8.47 & $\begin{array}{l}33.650 \\
82.000\end{array}$ & \\
\hline 7 & $\begin{array}{l}\text { Hayward Brook } \\
\text { at Wayland, } \\
\text { Mass. }\end{array}$ & 2.86 & $\begin{array}{r}1964-68 \\
\ldots \ldots\end{array}$ & 1965 & 18 & $\begin{array}{l}7.57 \\
8.47\end{array}$ & $\begin{array}{l}48 \\
74\end{array}$ & ()$^{--}$ \\
\hline 8 & $\begin{array}{l}\text { Concord River } \\
\text { below River } \\
\text { Meadow Brook } \\
\text { at Lowell, Mass. }\end{array}$ & ${ }^{7} 3.12$ & $\begin{array}{r}1937-68 \\
-\end{array}$ & $\begin{array}{l}1955 \\
-\ldots\end{array}$ & 22 & $\begin{array}{l}8.97 \\
9.16\end{array}$ & $\begin{array}{l}84,540 \\
84,800\end{array}$ & $\left.{ }^{-}\right)^{-}$ \\
\hline 9 & $\begin{array}{l}\text { Merrimack River } \\
\text { below Concord } \\
\text { River at }\end{array}$ & 94,425 & $\begin{array}{c}1736-1968 \\
-\end{array}$ & 1936 & 23 & $\begin{array}{l}68.4 \\
51.15\end{array}$ & $\begin{array}{r}173,000 \\
10 \\
44,400\end{array}$ & - \\
\hline 10 & $\begin{array}{l}\text { Richardson Brook } \\
\text { near Lowell, } \\
\text { Mass. }\end{array}$ & $1 .: 2$ & $\begin{array}{c}1962-68 \\
-\ldots-\end{array}$ & 1962 & 18 & $\begin{array}{l}14.68 \\
14.44\end{array}$ & $\begin{array}{l}153 \\
185\end{array}$ & (3) \\
\hline 11 & $\begin{array}{l}\text { Spicket River at } \\
\text { Methuen, Mass. }\end{array}$ & 73.8 & $\ldots$ & - & 19 & - & 1,440 & (a) \\
\hline 12 & $\begin{array}{c}\text { Shawsheen River } \\
\text { near Wilming- } \\
\text { ton. Mass. }\end{array}$ & 3.51 & $1964-68$ & 1967 & 19 & $\begin{array}{l}6.72 \\
8.60\end{array}$ & $\begin{array}{r}495 \\
1,050\end{array}$ & $\left({ }^{3}\right)$ \\
\hline 13 & $\begin{array}{l}\text { East Meadow } \\
\text { River near }\end{array}$ & 1.83 & $1962-68$ & 1962 & 19 & $\begin{array}{l}5.6 \\
5.45\end{array}$ & $\begin{array}{l}190 \\
211\end{array}$ & (3) \\
\hline 14 & $\begin{array}{l}\text { Cobbler Brook } \\
\text { near Merrimac, } \\
\text { Mass. }\end{array}$ & .77 & $1962-68$ & 1962 & $18^{--}$ & $\begin{array}{r}9.30 \\
10.69\end{array}$ & $\begin{array}{r}66 \\
117\end{array}$ & $\left({ }^{3}\right)$ \\
\hline \multicolumn{9}{|c|}{ Parker River basin } \\
\hline 15 & $\begin{array}{l}\text { Parker River } \\
\text { tributary near } \\
\text { Georgetown, } \\
\text { Mass. }\end{array}$ & .65 & $\begin{array}{c}1964-68 \\
-\end{array}$ & $\begin{array}{r}1965 \\
1966 \\
--\end{array}$ & $18^{-}$ & 10.90 & $\begin{array}{l}24 \\
76\end{array}$ & $(\overline{3})$ \\
\hline 16 & $\begin{array}{c}\text { Parker River at } \\
\text { Byfield, Mass. }\end{array}$ & 21.6 & $\begin{array}{r}1946-68 \\
-\end{array}$ & 1958 & $19^{-}$ & $\begin{array}{l}5.99 \\
5.61\end{array}$ & $\begin{array}{l}479 \\
489\end{array}$ & $-\overline{25}$ \\
\hline \multicolumn{9}{|c|}{ Ipswich River basin } \\
\hline 17 & $\begin{array}{l}\text { Maple Meadow } \\
\text { Brook at } \\
\text { Wilmington, } \\
\text { Mass. }\end{array}$ & 3.99 & $1962-68$ & 1962 & 19 & $\begin{array}{l}5.33 \\
5.63\end{array}$ & $\begin{array}{l}103 \\
119\end{array}$ & ()$\left.^{3}\right)$ \\
\hline 18 & $\begin{array}{l}\text { Ipswich River at } \\
\text { South Middle- } \\
\text { ton, Mass. }\end{array}$ & 43.4 & $1937-68$ & 1962 & 19 & $\begin{array}{l}6.99 \\
7.09\end{array}$ & $\begin{array}{l}808 \\
833\end{array}$ & 45 \\
\hline 19 & $\begin{array}{l}\text { Ipswich River near } \\
\text { Ipswich, Mass. }\end{array}$ & 124 & $\begin{array}{c}1887-1968 \\
-\end{array}$ & $\begin{array}{l}1936 \\
-\end{array}$ & 20,21 & $\begin{array}{l}7.70 \\
8.41\end{array}$ & $\begin{array}{l}2,610 \\
2,680\end{array}$ & 50 \\
\hline \multicolumn{9}{|c|}{ Mystic River basin } \\
\hline 20 & $\begin{array}{l}\text { Aberjona River at } \\
\text { Winchester, } \\
\text { Mass. }\end{array}$ & 23.3 & $1887-1968$ & $\begin{array}{l}1955 \\
1962\end{array}$ & 19 & $\begin{array}{l}14.43 \\
13.73\end{array}$ & $\begin{array}{l}835 \\
649\end{array}$ & (3) \\
\hline
\end{tabular}

See footnotes at end of table. 
TABLE 3.-Flood stages and discharges, March 18-26 in eastern Massachusetts and Rhode Island-Continued

\begin{tabular}{|c|c|c|c|c|c|c|c|c|}
\hline \multirow{4}{*}{ No. } & \multirow{4}{*}{$\begin{array}{l}\text { Stream and place } \\
\text { of determination }\end{array}$} & \multirow{4}{*}{$\begin{array}{l}\text { Drainage } \\
\text { area } \\
\text { (sq } \mathrm{mi})\end{array}$} & \multicolumn{6}{|c|}{ Maximum floods } \\
\hline & & & \multirow{2}{*}{\multicolumn{2}{|c|}{$\begin{array}{l}\text { Known before } \\
\text { March } 1968\end{array}$}} & \multirow{3}{*}{$\underset{1968}{\text { March }}$} & \multirow{3}{*}{$\begin{array}{l}\text { Gage } \\
\text { height } \\
\text { (feet) }\end{array}$} & \multicolumn{2}{|c|}{ Discharge } \\
\hline & & & & & & & \multirow[b]{2}{*}{ Cfs } & \multirow{2}{*}{$\begin{array}{l}\text { Recur- } \\
\text { rence } \\
\text { interva } \\
\text { (years) }\end{array}$} \\
\hline & & & Period & Year & & & & \\
\hline \multicolumn{9}{|c|}{ Charles River basin } \\
\hline 21 & $\begin{array}{c}\text { Charles River at } \\
\text { Charles River }\end{array}$ & 184 & $1887-1968$ & 1955 & & 9.24 & $\begin{array}{l}3,220 \\
3,220\end{array}$ & 40 \\
\hline & Village, Mass. & & & - & $\begin{array}{r}21-22 \\
22\end{array}$ & 8.72 & & \\
\hline 22 & Mother Brook at & & $1932-68$ & 1955 & & $\begin{array}{l}92.90 \\
87.18\end{array}$ & $\begin{array}{r}970 \\
1,040\end{array}$ & - \\
\hline 23 & Charles River at & 211 & $1960-68$ & 1960 & 21 & 5.16 & 1,470 & 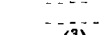 \\
\hline 24 & $\begin{array}{l}\text { Wellesley, Mass. } \\
\text { Charles River at }\end{array}$ & 12227 & $1887-1968$ & 1936 & 21 & 6.20 & $\begin{array}{l}2,410 \\
2,540\end{array}$ & \\
\hline & Waltham, Mass. & & & 1955 & $2 \overline{2}$ & $\begin{array}{r}5.35 \\
+5.39\end{array}$ & $(3)$ & $\ldots$ \\
\hline$\because \therefore$ & $\begin{array}{l}\text { Beaver Brook at } \\
\text { Belmont, Mass. }\end{array}$ & 4.09 & $1962-68$ & 1962 & 18 & $\begin{array}{l}12.46 \\
13.07\end{array}$ & $\begin{array}{l}200 \\
212\end{array}$ & $\left({ }^{3}\right)$ \\
\hline \multicolumn{9}{|c|}{ Neponset River basin } \\
\hline 26 & $\begin{array}{l}\text { Mill Brook at } \\
\text { Westwood, Mass. }\end{array}$ & 1.52 & $1964-68$ & $\begin{array}{l}1965 \\
1967\end{array}$ & $18^{\cdots}$ & $\begin{array}{r}7.05 \\
7.05 \\
10.12\end{array}$ & $\begin{array}{l}31 \\
31 \\
96\end{array}$ & $(3)$ \\
\hline 27 & Neponset River at & 35.2 & $1887-1968$ & $19 \overline{5} \overline{5}$ & & 14.65 & 1,490 & \\
\hline 28 & $\begin{array}{l}\text { Norwood, Mass. } \\
\text { East Branch }\end{array}$ & 27.2 & $1953-68$ & 1955 & 18 & $\begin{array}{r}10.48 \\
8.18\end{array}$ & $\begin{array}{l}1,140 \\
1,790\end{array}$ & 40 \\
\hline & $\begin{array}{l}\text { Neponset River } \\
\text { at Canton, Mass. }\end{array}$ & & +.... & $\ldots$ & 18 & 6.87 & 1,420 & 25 \\
\hline 29 & $\begin{array}{l}\text { Plantingfield Brook } \\
\text { at Norwood, } \\
\text { Mass. }\end{array}$ & 1.52 & $1964-68$ & $\begin{array}{l}1967 \\
-\end{array}$ & 18 & $\begin{array}{l}14.11 \\
15.06\end{array}$ & $\begin{array}{l}145 \\
178\end{array}$ & $\left({ }^{3}\right)$ \\
\hline 30 & $\begin{array}{l}\text { Neponset River } \\
\text { at Hyde Park, } \\
\text { Mass. }\end{array}$ & 97.8 & ...... & $\ldots$ & 20 & $\ldots$ & 1,550 & $\left({ }^{3}\right)$ \\
\hline \multicolumn{9}{|c|}{ Weymouth Back River basin } \\
\hline 31 & $\begin{array}{l}\text { Old Swamp River } \\
\text { near South }\end{array}$ & 4.29 & $1967-68$ & $\begin{array}{l}1967 \\
1967\end{array}$ & & 4.22 & 207 & $\ldots$ \\
\hline & $\begin{array}{l}\text { Weymouth, } \\
\text { Mass. }\end{array}$ & & -- & $\ldots$ & 18 & 5.22 & 566 & $\left({ }^{3}\right)$ \\
\hline \multicolumn{9}{|c|}{ North River basin } \\
\hline 32 & $\begin{array}{l}\text { Indian Head River } \\
\text { at Hanover, } \\
\text { Mass. }\end{array}$ & 30.3 & $1967-68$ & 1967 & $18-19$ & $\begin{array}{l}5.62 \\
7.13\end{array}$ & $\begin{array}{r}788 \\
1,390\end{array}$ & (a) \\
\hline \multicolumn{9}{|c|}{ South River basin } \\
\hline 33 & $\begin{array}{l}\text { Furnace Brook } \\
\text { near Marshfield, } \\
\text { Mass. }\end{array}$ & 1.61 & $1964-68$ & 1967 & 18 & $\begin{array}{l}9.41 \\
9.92\end{array}$ & $\begin{array}{l}54 \\
71\end{array}$ & $\left({ }^{3}\right)$ \\
\hline \multicolumn{9}{|c|}{ Jones River basin } \\
\hline 34 & $\begin{array}{l}\text { Jones River at } \\
\text { Kingston, Mass. }\end{array}$ & ${ }^{13} 15.8$ & $1967-68$ & $\begin{array}{l}1967 \\
1967\end{array}$ & & $1+4.65$ & 358 & \\
\hline & & & $\ldots$. & $\ldots$ & 19 & ${ }^{1+} 4.60$ & 15575 & $(3)$ \\
\hline \multicolumn{9}{|c|}{ Herring River basin } \\
\hline 35 & $\begin{array}{l}\text { Herring River at } \\
\text { North Hard- } \\
\text { wich, Mass. }\end{array}$ & 9.4 & $\begin{array}{r}1967-68 \\
-\ldots\end{array}$ & $\begin{array}{l}1967 \\
-\end{array}$ & 21 & $\begin{array}{l}4.88 \\
4.03\end{array}$ & $\begin{array}{l}54 \\
44\end{array}$ & $(3)$ \\
\hline \multicolumn{9}{|c|}{ East Branch Westport River basin } \\
\hline 36 & $\begin{array}{l}\text { Kirby Brook near } \\
\text { head of West- } \\
\text { port, Mass. }\end{array}$ & 3.69 & $\begin{array}{r}1964-68 \\
-\ldots\end{array}$ & 1967 & $\overline{18}$ & $\begin{array}{l}10.07 \\
12.16\end{array}$ & $\begin{array}{l}213 \\
378\end{array}$ & $\left({ }^{3}\right)$ \\
\hline
\end{tabular}

See footnotes at end of table. 
TABLE 3.-Flood stages and discharges, March 18-26 in eastern Massachusetts and Rhode Island-Continued

\begin{tabular}{|c|c|c|c|c|c|c|c|c|}
\hline \multirow{4}{*}{ No. } & \multirow{4}{*}{$\begin{array}{l}\text { Stream and place } \\
\text { of determination }\end{array}$} & \multirow{4}{*}{$\begin{array}{l}\text { Drainage } \\
\text { area } \\
\text { (sq mi) }\end{array}$} & \multicolumn{6}{|c|}{ Maximum floods } \\
\hline & & & \multirow{2}{*}{\multicolumn{2}{|c|}{$\begin{array}{l}\text { Known before } \\
\text { March } 1968\end{array}$}} & \multirow{3}{*}{$\begin{array}{c}\text { March } \\
1968\end{array}$} & \multirow{3}{*}{$\begin{array}{l}\text { Gage } \\
\text { height } \\
\text { (feet) }\end{array}$} & \multicolumn{2}{|c|}{ Discharge } \\
\hline & & & & & & & \multirow[b]{2}{*}{ Cfs } & \multirow{2}{*}{$\begin{array}{c}\text { Recur- } \\
\text { rence } \\
\text { interval } \\
\text { (years) }\end{array}$} \\
\hline & & & Period & Year & & & & \\
\hline \multicolumn{9}{|c|}{ West Branch Westport River hasin } \\
\hline 37 & $\begin{array}{l}\text { Adamsville } \\
\text { Brook at } \\
\text { Adamsville, R.I. }\end{array}$ & 8.6 & $\begin{array}{c}1941-68 \\
-\end{array}$ & 1960 & 18 & $\begin{array}{l}7.14 \\
7.70\end{array}$ & $\begin{array}{l}273 \\
221\end{array}$ & 6 \\
\hline \multicolumn{9}{|c|}{ Cold Brook basin } \\
\hline 38 & $\begin{array}{l}\text { Cold Brook near } \\
\text { Adamsville, R.I. }\end{array}$ & 1.15 & $\begin{array}{r}1966-68 \\
-\end{array}$ & 1967 & 18 & $\begin{array}{r}8.93 \\
11.19 \\
\end{array}$ & $\begin{array}{l}47 \\
79 \\
\end{array}$ & $\left({ }^{3}\right)$ \\
\hline \multicolumn{9}{|c|}{ Taunton River basin } \\
\hline 39 & $\begin{array}{l}\text { Meadow Brook } \\
\text { tributary near } \\
\text { Whitman, Mass. }\end{array}$ & 1.04 & $\begin{array}{c}1967-68 \\
-\cdots\end{array}$ & 1967 & 18 & $\begin{array}{r}9.73 \\
10.96\end{array}$ & $\begin{array}{l}49 \\
56\end{array}$ & $\left({ }^{3}\right)$ \\
\hline 40 & $\begin{array}{l}\text { Dorchester Brook } \\
\text { near Brockton, } \\
\text { Mass. }\end{array}$ & 4.67 & $1962-68$ & $\begin{array}{l}1962 \\
1965\end{array}$ & $18^{-\cdots}$ & $\begin{array}{r}16.75 \\
5.86\end{array}$ & $\begin{array}{r}130 \\
359\end{array}$ & $\left({ }^{3}\right)$ \\
\hline 41 & $\begin{array}{l}\text { Taunton River at } \\
\text { State Farm, } \\
\text { Mass. }\end{array}$ & 260 & $1930-68$ & 1955 & 20 & $\begin{array}{l}13.02 \\
14.48\end{array}$ & $\begin{array}{l}4,010 \\
4,980\end{array}$ & 51.2 \\
\hline 42 & $\begin{array}{l}\text { Snows Brook near } \\
\text { Bridgewater, } \\
\text { Mass. }\end{array}$ & 1.41 & $1964-68$ & 1964 & 18 & $\begin{array}{r}5.75 \\
10.09\end{array}$ & $\begin{array}{r}29 \\
153\end{array}$ & $\left({ }^{3}\right)$ \\
\hline 43 & $\begin{array}{l}\text { Wading River at } \\
\text { West Mansfleld, } \\
\text { Mass. }\end{array}$ & 19.2 & $\begin{array}{r}1954-68 \\
-\ldots\end{array}$ & 1955 & $19^{-\cdots}=$ & $\begin{array}{l}6.22 \\
6.60\end{array}$ & $\begin{array}{l}519 \\
541\end{array}$ & $\left({ }^{3}\right)$ \\
\hline 44 & $\begin{array}{l}\text { Wading River } \\
\text { near Norton, } \\
\text { Mass. }\end{array}$ & 42.4 & $\begin{array}{r}1926-68 \\
-\end{array}$ & 1955 & 19 & $\begin{array}{l}10.98 \\
11.47\end{array}$ & $\begin{array}{l}1,170 \\
1,460\end{array}$ & 51.2 \\
\hline 45 & $\begin{array}{l}\text { Threemile River } \\
\text { tributary near } \\
\text { Oakland, Mass. }\end{array}$ & .50 & $1964-68$ & 1967 & $18^{-}$ & $\begin{array}{l}6.58 \\
7.13\end{array}$ & $\begin{array}{l}14 \\
18\end{array}$ & $\left({ }^{3}\right)$ \\
\hline 46 & $\begin{array}{l}\text { Threemile River } \\
\text { at North } \\
\text { Dighton, Mass. }\end{array}$ & 83.8 & $\begin{array}{r}1967-68 \\
-\ldots\end{array}$ & $\begin{array}{l}1967 \\
--\end{array}$ & $19^{\cdots}$ & $\begin{array}{l}6.31 \\
8.30\end{array}$ & $\begin{array}{l}1,340 \\
2,490\end{array}$ & $\left({ }^{3}\right)$ \\
\hline 47 & $\begin{array}{l}\text { Segreganset River } \\
\text { near Dighton, } \\
\text { Mass. }\end{array}$ & 10.6 & $1967-68$ & 1967 & 18 & $\begin{array}{l}5.81 \\
7.51\end{array}$ & $\begin{array}{l}515 \\
867\end{array}$ & $\left(3^{3}\right)$ \\
\hline 48 & $\begin{array}{l}\text { Holloway Brook } \\
\text { near Myricks, } \\
\text { Mass. }\end{array}$ & 1.16 & $1967-68$ & 1967 & $18^{--}$ & $\begin{array}{l}6.93 \\
7.47\end{array}$ & $\begin{array}{l}68 \\
86\end{array}$ & $\left({ }^{3}\right)$ \\
\hline 49 & $\begin{array}{l}\text { Quaker Brook } \\
\text { near Myricks, }\end{array}$ & 1.94 & $\begin{array}{r}1967-68 \\
\ldots\end{array}$ & 1967 & $18^{\cdots-}$ & $\begin{array}{l}10.02 \\
10.78\end{array}$ & $\begin{array}{l}156 \\
190\end{array}$ & $\left({ }^{3}\right)$ \\
\hline 50 & $\begin{array}{l}\text { Taunton River } \\
\text { tributary near } \\
\text { Fall River, } \\
\text { Mass. }\end{array}$ & .25 & $\begin{array}{r}1964-68 \\
-\end{array}$ & 1966 & $18^{--}$ & $\begin{array}{l}11.06 \\
11.23\end{array}$ & $\begin{array}{l}49 \\
53\end{array}$ & $\left({ }^{3}\right)$ \\
\hline
\end{tabular}

\begin{tabular}{|c|c|c|c|c|c|c|c|c|}
\hline \multicolumn{9}{|c|}{ Palmer River basin } \\
\hline 51 & $\begin{array}{l}\text { Bliss Brook near } \\
\text { Rehoboth, Mass. }\end{array}$ & 4.96 & $1963-68$ & 1967 & 18 & $\begin{array}{l}5.04 \\
5.43\end{array}$ & $\begin{array}{l}251 \\
441\end{array}$ & $\left({ }^{3}\right)$ \\
\hline \multicolumn{9}{|c|}{ Runnins River basin } \\
\hline 52 & $\begin{array}{l}\text { Runnins River at } \\
\text { Seekonk, Mass. }\end{array}$ & 4.24 & $\begin{array}{c}1967-68 \\
-\end{array}$ & 1967 & 18 & $\begin{array}{l}6.50 \\
7.50\end{array}$ & $\begin{array}{r}94 \\
155\end{array}$ & $\left({ }^{3}\right)$ \\
\hline \multicolumn{9}{|c|}{ Metacomet Brook basin } \\
\hline 53 & $\begin{array}{l}\text { Metacomet Brook } \\
\text { at East } \\
\text { Providence, } \\
\text { Mass. }\end{array}$ & .82 & $\begin{array}{r}1966-68 \\
-\ldots\end{array}$ & 1967 & 18 & $\begin{array}{r}11.05 \\
8.11\end{array}$ & $\begin{array}{r}225 \\
81\end{array}$ & $(3)$ \\
\hline
\end{tabular}

Blackst one River basin

\begin{tabular}{ccccccc}
\hline $54 \quad \begin{array}{l}\text { Kettle Brook at } \\
\text { Worcester, Mass. }\end{array}$ & 31.3 & $1924-68$ & 1955 & 18 & 12.78 & $\begin{array}{r}3,970 \\
\end{array}$ \\
& & $-\ldots-100$
\end{tabular}

See footnotes at end of table. 
TABLE 3.-Flood stages and discharges, March 18-26 in eastern Massachusetts and Rhode Island-Continued

\begin{tabular}{|c|c|c|c|c|c|c|c|c|}
\hline \multirow{5}{*}{ No. } & \multirow{5}{*}{$\begin{array}{l}\text { Stream and place } \\
\text { of determination }\end{array}$} & \multirow{4}{*}{$\begin{array}{l}\text { Drainage } \\
\text { area } \\
\text { (sq mi) }\end{array}$} & \multicolumn{6}{|c|}{ Maximum floods } \\
\hline & & & \multirow{2}{*}{\multicolumn{2}{|c|}{$\begin{array}{l}\text { Known before } \\
\text { March } 1968\end{array}$}} & \multirow{3}{*}{$\underset{1968}{\operatorname{March}}$} & \multirow{3}{*}{$\begin{array}{l}\text { Gage } \\
\text { height } \\
\text { (feet) }\end{array}$} & \multicolumn{2}{|c|}{ Discharge } \\
\hline & & & & & & & \multirow{3}{*}{ Cfs } & \multirow{3}{*}{$\begin{array}{l}\text { Recur- } \\
\text { rence } \\
\text { interval } \\
\text { (years) }\end{array}$} \\
\hline & & & Period & Year & & & & \\
\hline & & \multicolumn{5}{|c|}{ Blackstone River basin-Continued } & & \\
\hline 55 & $\begin{array}{l}\text { Quinsigamond } \\
\text { River at North } \\
\text { Grafton, Mass. }\end{array}$ & 25.5 & $\begin{array}{r}1940-68 \\
-\ldots\end{array}$ & 1955 & 19 & $\begin{array}{l}5.15 \\
4.03\end{array}$ & $\begin{array}{l}820 \\
452\end{array}$ & $\left(\mathbf{3}^{3}\right)$ \\
\hline 56 & $\begin{array}{l}\text { Blackstone River } \\
\text { at Northbridge, }\end{array}$ & 139 & $1901-68$ & $\begin{array}{l}1955 \\
1955\end{array}$ & & +17.53 & 16,900 & $\cdots$ \\
\hline 57 & $\begin{array}{l}\text { Mass. } \\
\text { West River below }\end{array}$ & 27.9 & $1962-68$ & $\overline{1962}$ & & $\begin{array}{r}10.90 \\
3.34\end{array}$ & $\begin{array}{r}4,760 \\
355\end{array}$ & $\left({ }^{3}\right)$ \\
\hline & $\begin{array}{l}\text { West Hill Dam, } \\
\text { near Uxbridge, } \\
\text { Mass. }\end{array}$ & 26.0 & & & 26 & $\begin{array}{l}0.04 \\
3.45\end{array}$ & 370 & (3) \\
\hline 58 & $\begin{array}{l}\text { Dry Arm Brook } \\
\text { near Wallum } \\
\text { Lake, R.I. }\end{array}$ & 1.74 & $\begin{array}{r}1966-68 \\
\cdots\end{array}$ & 1967 & $18^{-}$ & $\begin{array}{l}8.30 \\
9.72\end{array}$ & $\begin{array}{l}56 \\
92\end{array}$ & (a) \\
\hline 59 & $\begin{array}{l}\text { Nipmuc River } \\
\text { near Harrisville, } \\
\text { R.I. }\end{array}$ & 16.0 & $\begin{array}{c}1965-68 \\
-\ldots-\ldots\end{array}$ & $\begin{array}{l}1967 \\
-\cdots\end{array}$ & 18 & $\begin{array}{l}6.58 \\
7.42\end{array}$ & $\begin{array}{r}527 \\
1,020\end{array}$ & $\left({ }^{3}\right)$ \\
\hline 60 & $\begin{array}{l}\text { Chepachet River } \\
\text { at Chepachet, } \\
\text { R.I. }\end{array}$ & 17.4 & $\begin{array}{c}1965-68 \\
\ldots\end{array}$ & $\begin{array}{l}1967 \\
\cdots\end{array}$ & 18 & $\begin{array}{l}7.27 \\
8.85\end{array}$ & $\begin{array}{r}371 \\
1,080\end{array}$ & $(3)$ \\
\hline 61 & $\begin{array}{l}\text { Mowry Paine } \\
\text { Brook near } \\
\text { Chepachet, R.I. }\end{array}$ & 1.87 & $\begin{array}{c}1966-68 \\
-\cdots\end{array}$ & 1967 & $18^{--}$ & $\begin{array}{r}8.59 \\
10.24\end{array}$ & $\begin{array}{l}107 \\
360\end{array}$ & (a) \\
\hline 62 & $\begin{array}{l}\text { Branch River at } \\
\text { Forestdale, R.I. }\end{array}$ & 91.2 & $1887-1968$ & & 18 & $\overline{11.90}$ & $\begin{array}{l}5,800 \\
4,980\end{array}$ & $5_{1.1}$ \\
\hline 63 & $\begin{array}{l}\text { Bungay Brook near } \\
\text { Sheldonville, } \\
\text { Mass. }\end{array}$ & 2.66 & $1964-68$ & $19 \overline{6} \overline{7}$ & $18^{-}$ & $\begin{array}{l}6.99 \\
8.24\end{array}$ & $\begin{array}{r}4,500 \\
92 \\
188\end{array}$ & $\left({ }^{3}\right)$ \\
\hline 64 & $\begin{array}{l}\text { Mlackstone River } \\
\text { at Woonsocket, } \\
\text { R.I. }\end{array}$ & 416 & $1946-68$ & 1955 & 19 & $\begin{array}{l}21.8 \\
14.64\end{array}$ & $\begin{array}{c}1732,900 \\
15,400\end{array}$ & 25 \\
\hline 65 & $\begin{array}{l}\text { Blackstone River } \\
\text { tributary at } \\
\text { Woonsocket, } \\
\text { R.I. }\end{array}$ & 2.22 & $1966-68$ & 1967 & $18^{\cdots}$ & $\begin{array}{l}2.97 \\
3.52\end{array}$ & $\begin{array}{l}116 \\
285\end{array}$ & $\left({ }^{3}\right)$ \\
\hline 66 & $\begin{array}{l}\text { Blackstone River } \\
\text { tributary No. } 2 \\
\text { at Berkeley, R.I. }\end{array}$ & 1.04 & $1966-68$ & $\begin{array}{l}1967 \\
-\cdots\end{array}$ & $18^{--}$ & $\begin{array}{l}6.09 \\
5.80\end{array}$ & $\begin{array}{l}34 \\
27\end{array}$ & $(3)$ \\
\hline \multicolumn{9}{|c|}{ Moshassuck River basin } \\
\hline 67 & $\begin{array}{l}\text { Moshassuck River } \\
\text { Providence, R.I. }\end{array}$ & 23.1 & $1964-68$ & 1967 & 18 & $\begin{array}{l}4.40 \\
4.34\end{array}$ & $\begin{array}{l}1,110 \\
2,390\end{array}$ & $(3)$ \\
\hline 68 & $\begin{array}{l}\text { Woonasquatucket } \\
\text { River at }\end{array}$ & 38.3 & 1936 & 1954 & & $\begin{array}{l}4.04 \\
7.03\end{array}$ & 1,100 & \\
\hline & Centerdale, R.I. & & $1942-68$ & $\ldots$ & 18 & 7.75 & 1,440 & ${ }^{5} 1.1$ \\
\hline \multicolumn{9}{|c|}{ Pawtuxet River basin } \\
\hline 69 & $\begin{array}{l}\text { Mosquitohawk } \\
\text { Brook near } \\
\text { North Scituate, } \\
\text { R.I. }\end{array}$ & 3.06 & $1966-68$ & 1967 & $18^{--}$ & $\begin{array}{l}3.09 \\
3.40\end{array}$ & $\begin{array}{l}293 \\
630\end{array}$ & $\left({ }^{3}\right)$ \\
\hline 70 & $\begin{array}{l}\text { Shippee Brook } \\
\text { tributary at } \\
\text { North Foster, } \\
\text { R.I. }\end{array}$ & .55 & $1966-68$ & $\begin{array}{l}1967 \\
-\ldots\end{array}$ & $18^{-}$ & $\begin{array}{l}8.74 \\
9.87\end{array}$ & $\begin{array}{l}21 \\
38\end{array}$ & $\left({ }^{3}\right)$ \\
\hline 71 & $\begin{array}{l}\text { Wilbur Hollow } \\
\text { Brook near } \\
\text { Clayville, R.I. }\end{array}$ & 4.61 & $\begin{array}{c}1966-68 \\
\ldots\end{array}$ & 1967 & 18 & $\begin{array}{l}12.20 \\
14.40\end{array}$ & $\begin{array}{l}128 \\
300\end{array}$ & $-\overline{(3)}$ \\
\hline 72 & $\begin{array}{l}\text { Nooseneck River } \\
\text { at Nooseneck } \\
\text { R.I. }\end{array}$ & 8.23 & $\begin{array}{c}1964-68 \\
-\ldots\end{array}$ & 1965 & 18 & $\begin{array}{l}4.65 \\
6.13\end{array}$ & $\begin{array}{l}223 \\
318\end{array}$ & $\left({ }^{3}\right)$ \\
\hline 73 & Carr River near & 6.73 & $1964-68$ & 1965 & $18-$ & $\begin{array}{l}4.77 \\
6.50\end{array}$ & $\begin{array}{r}96 \\
221\end{array}$ & $(3)$ \\
\hline 74 & Bear Creek near & 3.98 & $1966-68$ & 1967 & & 8.59 & 110 & \\
\hline 75 & $\begin{array}{l}\text { Coventry, R.I. } \\
\text { South Branch }\end{array}$ & 63.8 & 1936 & 1936 & 18 & 10.11 & $\begin{array}{r}118 \\
1,810\end{array}$ & $\cdots$ \\
\hline & $\begin{array}{l}\text { atwashington, } \\
\text { R.I. }\end{array}$ & & 341-b8. & $\ldots$ & $18-19$ & 5.13 & 1,860 & $\left({ }^{3}\right)$ \\
\hline
\end{tabular}

See footnotes at end of table. 
TABLE 3.-Flood stages and discharges, March 18-26 in eastern Massachusetts and Rhode Island-Continued

\begin{tabular}{|c|c|c|c|c|c|c|c|c|}
\hline \multirow{4}{*}{ No. } & \multirow{4}{*}{$\begin{array}{l}\text { Stream and place } \\
\text { of determination }\end{array}$} & \multirow{4}{*}{$\begin{array}{l}\text { Drainage } \\
\text { area } \\
\text { (sq mi) }\end{array}$} & \multicolumn{6}{|c|}{ Maximum floods } \\
\hline & & & \multirow{2}{*}{\multicolumn{2}{|c|}{$\begin{array}{l}\text { Known before } \\
\text { March } 1968\end{array}$}} & \multirow{3}{*}{$\underset{1968}{\operatorname{March}}$} & \multirow{3}{*}{$\begin{array}{l}\text { Gage } \\
\text { height } \\
\text { (feet) }\end{array}$} & \multicolumn{2}{|c|}{ Discharge } \\
\hline & & & & & & & & \\
\hline & & & Period & Year & & & Cfs & $\begin{array}{c}\text { interval } \\
\text { (years) } \\
\end{array}$ \\
\hline \multicolumn{9}{|c|}{ Pawtuxet River basin-Continued } \\
\hline 76 & $\begin{array}{l}\text { Furnace Hill } \\
\text { Brook at } \\
\text { Cranston, R.I. }\end{array}$ & 4.19 & $1966-68$ & $\begin{array}{l}1967 \\
-\ldots-\end{array}$ & 18 & $\begin{array}{l}4.58 \\
4.67\end{array}$ & $\begin{array}{l}516 \\
586\end{array}$ & ()$^{3}$ \\
\hline 77 & $\begin{array}{l}\text { Pawtuxet River } \\
\text { at Cranston, R.I. }\end{array}$ & 200 & $1940-68$ & 1967 & $18-19$ & $\begin{array}{r}9.95 \\
11.53\end{array}$ & 2,480 & $\ldots$ \\
\hline 78 & $\begin{array}{l}\text { Pocasset River } \\
\text { near North } \\
\text { Scituate, R.I. }\end{array}$ & 1.34 & $1966-68$ & 1966 & $18^{--19}$ & $\begin{array}{l}7.63 \\
9.29\end{array}$ & $\begin{array}{r}0,15 \\
15 \\
55\end{array}$ & $\left(3^{3}\right)$ \\
\hline \multicolumn{9}{|c|}{ Hardig River basin } \\
\hline 79 & $\begin{array}{l}\text { Hardig Brook near } \\
\text { West Warwick, } \\
\text { R.I. }\end{array}$ & 3.16 & $1966-68$ & 1967 & $18^{-\cdots}$ & $\begin{array}{l}4.49 \\
5.49\end{array}$ & $\begin{array}{l}160 \\
300\end{array}$ & $\left({ }^{3}\right)$ \\
\hline \multicolumn{9}{|c|}{ Potowomut River basin } \\
\hline 80 & $\begin{array}{l}\text { Frenchtown } \\
\text { Brook near } \\
\text { Davisville, R.I. }\end{array}$ & 3.12 & $1966-68$ & 1966 & 18 & $\begin{array}{l}4.16 \\
6.86\end{array}$ & $\begin{array}{r}41 \\
118\end{array}$ & $\left({ }^{3}\right)$ \\
\hline 81 & $\begin{array}{l}\text { Potowomut River } \\
\text { near East } \\
\text { Greenwich, R.I. }\end{array}$ & 23.0 & $\begin{array}{r}1938-68 \\
1941-68 \\
-\end{array}$ & $\begin{array}{l}1938 \\
1962 \\
- \\
\end{array}$ & $18^{-1}$ & $\begin{array}{c}158.5 \\
3.36 \\
\end{array}$ & $\begin{array}{l}482 \\
866 \\
\end{array}$ & ${ }^{5} 1.2$ \\
\hline \multicolumn{9}{|c|}{ Smelt Brook basin } \\
\hline 82 & $\begin{array}{c}\text { Browns Brook at } \\
\text { Wakefield, R.I. }\end{array}$ & .50 & $\begin{array}{c}1966-68 \\
\cdots\end{array}$ & $\begin{array}{r}1966 \\
-\ldots \\
\end{array}$ & $18^{---}$ & $\begin{array}{r}8.15 \\
10.53 \\
\end{array}$ & $\begin{array}{l}11 \\
19 \\
\end{array}$ & $\left({ }^{3}\right)$ \\
\hline \multicolumn{9}{|c|}{ Pawcatuek River basin } \\
\hline 83 & $\begin{array}{l}\text { Glen Rock Brook } \\
\text { tributary at } \\
\text { Usquepaug, R.I. }\end{array}$ & 1.04 & $1966-68$ & 1966 & 18 & $\begin{array}{l}5.32 \\
6.51\end{array}$ & $\begin{array}{r}57 \\
104\end{array}$ & $\left({ }^{3}\right)$ \\
\hline 84 & $\begin{array}{l}\text { Pawcatuck River } \\
\text { at Wood River } \\
\text { Junction, R.I. } \\
\text { Meadow Brook }\end{array}$ & 100 & $1941-68$ & $\begin{array}{l}1954 \\
1962 \\
1967\end{array}$ & $19^{-\cdots}$ & $\begin{array}{r}6.23 \\
7.80 \\
4.25\end{array}$ & $\begin{array}{r}1,130 \\
1,700 \\
\mathbf{5 5}\end{array}$ & 51.1 \\
\hline 85 & $\begin{array}{l}\text { Meadow Brook } \\
\text { near Carolina, } \\
\text { R.I. }\end{array}$ & 5.53 & $1966-68$ & 1967 & $18^{-\cdots}$ & $\begin{array}{l}4.25 \\
6.07\end{array}$ & $\begin{array}{r}55 \\
164\end{array}$ & $\left({ }^{3}\right)$ \\
\hline 86 & $\begin{array}{l}\text { Wood River near } \\
\text { Arcadia, R.I. }\end{array}$ & 35.2 & $1964-68$ & 1964 & 18 & $\begin{array}{r}\left({ }^{3}\right) \\
8.64\end{array}$ & $\begin{array}{l}390 \\
896\end{array}$ & $\left({ }^{3}\right)$ \\
\hline 87 & $\begin{array}{l}\text { Wood River } \\
\text { tributary near } \\
\text { Arcadia, R.I. }\end{array}$ & .77 & $1966-68$ & 1967 & & $\begin{array}{r}8.05 \\
8.87\end{array}$ & $\begin{array}{l}11 \\
19\end{array}$ & $\left({ }^{3}\right)$ \\
\hline 88 & $\begin{array}{l}\text { Wood River at } \\
\text { Hope Valley, R.I. }\end{array}$ & 72.4 & $\begin{array}{l}1711-1968 \\
1936, \\
1942-68 .\end{array}$ & $\begin{array}{l}1886 \\
1936\end{array}$ & & ${ }^{19} 12.4$ & $\stackrel{(3)}{1,540}^{-1}$ & $\ldots$ \\
\hline 89 & $\begin{array}{l}\text { Perry Healy Brook } \\
\text { near Bradford, } \\
\text { R.I. }\end{array}$ & 1.82 & $1966-68$ & 1967 & $\begin{array}{l}18-19 \\
j 8^{-\cdots}\end{array}$ & $\begin{array}{r}8.26 \\
8.15 \\
10.52\end{array}$ & $\begin{array}{r}1,720 \\
47 \\
108\end{array}$ & $\begin{array}{r}45 \\
-\overline{(3)}\end{array}$ \\
\hline 90 & $\begin{array}{l}\text { Pawcatuck River } \\
\text { at Westerly, R.I. }\end{array}$ & 295 & $\begin{array}{c}1635-1968 \\
1887-1968 \\
-\end{array}$ & $\begin{array}{l}1938 \\
1927 \\
--\end{array}$ & 18 & $\begin{array}{l}\text { 1s } 15.0 \\
10.49\end{array}$ & $\begin{array}{c}\left({ }^{3}\right) \\
(20) \\
4,470\end{array}$ & $\overline{5} 1.1$ \\
\hline \multicolumn{9}{|c|}{ Thames River basin } \\
\hline 91 & $\begin{array}{l}\text { Browns Brook } \\
\text { near Webster, } \\
\text { Mass. }\end{array}$ & .49 & $1963-68$ & 1967 & 18 & $\begin{array}{l}2.01 \\
2.46\end{array}$ & $\begin{array}{r}19 \\
125\end{array}$ & $\left({ }^{3}\right)$ \\
\hline 9 & $\begin{array}{l}\text { Bucks Horn Brook } \\
\text { at Greene, R.I. }\end{array}$ & 5.52 & $\begin{array}{c}1966-68 \\
-\end{array}$ & $\begin{array}{l}1967 \\
\cdots-\end{array}$ & 18 & $\begin{array}{l}3.76 \\
5.49\end{array}$ & $\begin{array}{l}144 \\
353\end{array}$ & $\left({ }^{3}\right)$ \\
\hline
\end{tabular}

1 Net above gage; total above gage, $433 \mathrm{sq} \mathrm{mi} \mathrm{(fiow} \mathrm{diverted} \mathrm{from} 117 \mathrm{sq} \mathrm{mi}$ for use of Boston metropolitan district and city of Worchester).

2 Includes water wasted in diverting drainage from $117 \mathrm{sq} \mathrm{mi}$ in basin of South Branch Nashua River.

${ }_{3}$ Not determined.

4 Affected by backwater from debris.

5 Ratio of peak discharge to 50 -year flood.

- Maximum daily. 
that of the great winter flood of 1886. At Charles River Village, Mass., on the Charles River (No. 21, table 3) the March peak equaled that of August 1955, both of which were greater than any other since at least 1886 . Many streams reached peaks having recurrence intervals greater than 50 years. Streams such as Wading and Taunton Rivers in southeastern Massachusetts and Potowomut and Pawcatuck Rivers, among others in Rhode Island, had peak flows of this magnitude.

Damage from the flooding was great, but there was no loss of life. Thousands of homes suffered damage, and industrial, commercial, and public losses were severe. The Corps of Engineers estimated that losses in Massachusetts amounted to about $\$ 28$ million and in Rhode Island, $\$ 9$ million. Damage to dwellings exceeded all other losses. The Corps indicated that the major factor in the size of residential losses was the development of wetland areas over the years. Encroachment of stream channels in urban centers was also a contributing factor in overall losses.

\section{FLOODS OF APRIL 8-11 IN WEST-CENTRAL LOUISIANA}

By Fred N. LeE

An almost stationary line of thunderstorms from 50 to 75 miles wide caused widespread flooding in west-central Louisiana on April 8-11. Two cells of intense rainfall occurred along this line of thunderstorms, one in the Many-Robeline area and the other in the Montgomery area (fig. 5). Unofficial reports from Burmuda, near Montgomery, and from Fort Jesup, near Many, indicated rainfall of 14.0 inches, and the U.S. Weather Bureau tion at Many reported rainfall of 12.62 inches. Several other stations reported rainfall in excess of 8.0 inches.

At five discharge stations and at two crest-stage stations the peak discharge exceeded the known maximum for the period of record. At five of these stations the peak discharge exceeded the 50 -year flood. Table 4 is a list of the stations in west-central Louisiana where the discharge equaled or exceded a 4-year flood.

Flood damage was estimated at $\$ 958,000$. About 80,000 acres

7 Net above gage; total above gage, $405 \mathrm{sq} \mathrm{mi}$.

8 Includes water wasted from $92.6 \mathrm{sq} \mathrm{mi} \mathrm{in} \mathrm{basins} \mathrm{of} \mathrm{Sudbury} \mathrm{River} \mathrm{and} \mathrm{Lake} \mathrm{Cochituate}$

Net above gage; total above gage, $4,635 \mathrm{sq} \mathrm{mi}$.

${ }^{10}$ Includes water wasted from $210 \mathrm{sq} \mathrm{mi}$ in basins of Sudbury and South Branch Nashua Rivers and Lake Cochituate.

${ }^{11}$ Affected by backwater from Upper Mystic Lake.

12 Excludes $23.6 \mathrm{sq}$ mi drained by Stony Brook, from which flow is diverted for municinal supply of Cambridge.

${ }_{13}$ Excludes $4.09 \mathrm{sq} \mathrm{mi}$ above outlet of Silver Lake.

14 Affected by backwater from tide.

15 Includes water wasted from $4.09 \mathrm{sq} \mathrm{mi}$ above outlet of Silver Lake

16 Affected by ice jam.

17 Affected by failure of dam on Mill River.

18 Affected by backwater from hurricane tidal wave.

${ }^{10}$ At least.

20 Possibly more than $6,300 \mathrm{cfs}$. 


\section{9}

Flood-determination point Number croressponds to that in table 4

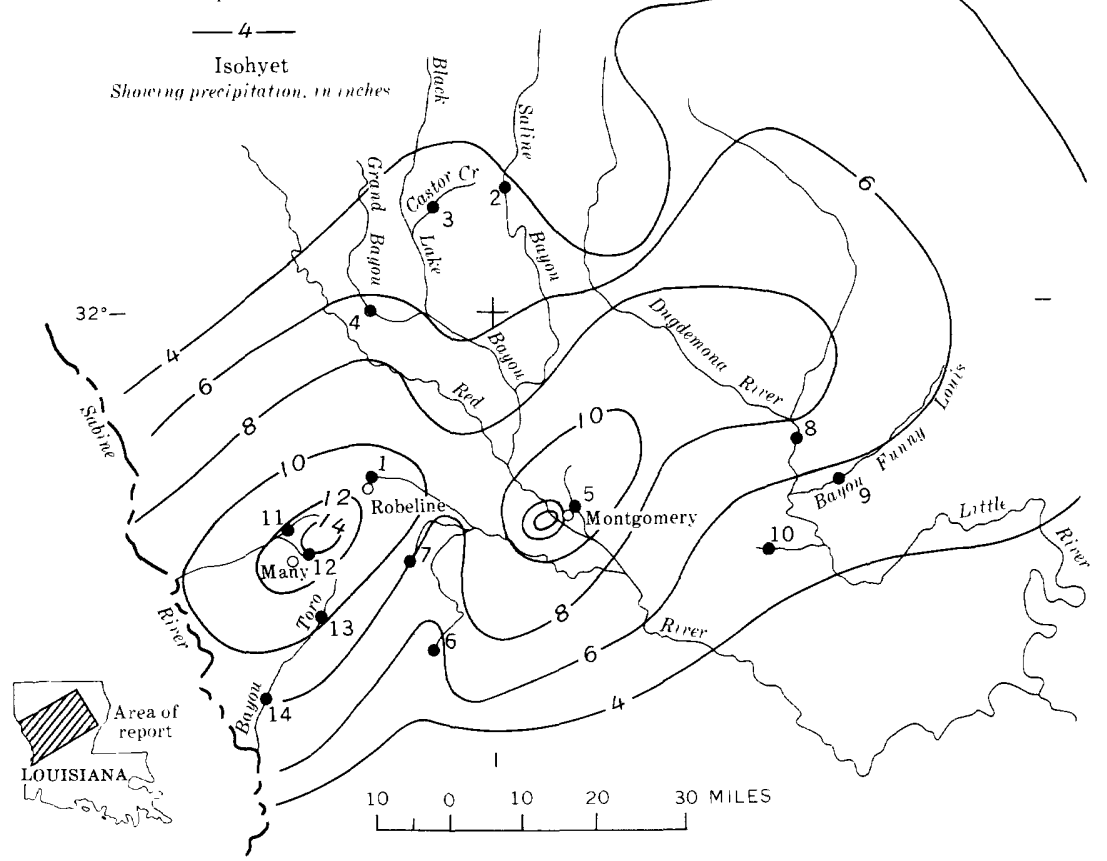

FIGURE 5.--Flood area; location of flood-determination points, and isohyets for April 8-10, floods of April 8-11 in west-central Louisiana.

in low-lying areas were inundated. Many crops were washed out or drowned and planting was delayed. Some bridges on rural roads were washed out and main highways were closed. About 150 families were evacuated in Natchitoches, Sabine, and Winn Parishes.

TABLE 4.-Fiood stages and discharges, April 8-11 in west-central Louisiana

\begin{tabular}{|c|c|c|c|c|c|c|c|c|}
\hline \multirow{4}{*}{ No. } & \multirow{4}{*}{$\begin{array}{l}\text { Stream and place } \\
\text { of determination }\end{array}$} & \multirow{4}{*}{$\begin{array}{c}\text { Drainage } \\
\text { area } \\
(\text { sq mi) }\end{array}$} & \multicolumn{6}{|c|}{ Maximum floods } \\
\hline & & & \multirow{2}{*}{\multicolumn{2}{|c|}{$\begin{array}{c}\text { Known before } \\
\text { April } 1968\end{array}$}} & \multirow{3}{*}{$\begin{array}{l}\text { April } \\
1968\end{array}$} & \multirow{3}{*}{$\begin{array}{c}\text { Gage } \\
\text { height } \\
\text { (feet) }\end{array}$} & \multicolumn{2}{|c|}{ Discharge } \\
\hline & & & & & & & \multirow[b]{2}{*}{ Cfs } & \multirow{2}{*}{$\begin{array}{c}\text { Recul- } \\
\text { rence } \\
\text { interva } \\
\text { (years) }\end{array}$} \\
\hline & & & Period & Year & & & & \\
\hline \multicolumn{9}{|c|}{ Red River basin } \\
\hline 1 & \multirow{3}{*}{$\begin{array}{l}\text { Bayou Dupont } \\
\text { near Robeline. } \\
\text { Saline Bayou } \\
\text { near Lucky. } \\
\text { Castor Creek } \\
\text { at Castor. }\end{array}$} & 35.1 & $1957-68$ & 1961 & \multirow{3}{*}{$\begin{array}{l}8 \\
9 \\
8^{-}\end{array}$} & \multirow{3}{*}{$\begin{aligned} 10.24 \\
11.70 \\
12.9 \\
9.49 \\
48.70 \\
48.23\end{aligned}$} & \multirow{3}{*}{$\begin{array}{r}\mathbf{5 , 3 0 0} \\
13,000 \\
13,500 \\
5,080 \\
(2) \\
2,250\end{array}$} & $\begin{array}{ll}1 & 1.5\end{array}$ \\
\hline 2 & & 154 & $1940-68$ & 1945 & & & & $5^{\cdots}$ \\
\hline 3 & & 27.9 & $\begin{array}{l}1954-68 \\
-\quad \ldots\end{array}$ & 1955 & & & & 12 \\
\hline
\end{tabular}

See footnotes at end of table. 
TABLE 4.-Flood stages and discharges, April 8-11 in west-central LouisianaContinued

\begin{tabular}{|c|c|c|c|c|c|c|c|c|}
\hline \multirow{4}{*}{ No. } & \multirow{4}{*}{$\begin{array}{l}\text { Stream and place } \\
\text { of determination }\end{array}$} & \multirow{4}{*}{$\begin{array}{c}\text { Drainage } \\
\text { area } \\
\text { (sq mi) }\end{array}$} & \multicolumn{6}{|c|}{ Maximum floods } \\
\hline & & & \multirow{2}{*}{\multicolumn{2}{|c|}{$\begin{array}{l}\text { Known before } \\
\text { April } 1968\end{array}$}} & \multirow{3}{*}{$\begin{array}{l}\text { April } \\
1968\end{array}$} & \multirow{3}{*}{$\begin{array}{c}\text { Gage } \\
\text { height } \\
\text { (feet) }\end{array}$} & \multicolumn{2}{|c|}{ Discharge } \\
\hline & & & & & & & \multirow[b]{2}{*}{ Cfs } & \multirow{2}{*}{$\begin{array}{c}\text { Recur- } \\
\text { rence } \\
\text { interval } \\
\text { (years) }\end{array}$} \\
\hline & & & Period & Year & & & & \\
\hline \multicolumn{9}{|c|}{ Red River basin-Continued } \\
\hline 4 & Grand Bayou & 93.9 & $1957-68$ & 1958 & & 11.47 & 7,920 & \\
\hline 5 & $\begin{array}{l}\text { near Gousnatia. } \\
\text { antachie Creek } \\
\text { near Montgom- } \\
\text { ery. }\end{array}$ & 47 & $1942-68$ & 1953 & 8 & $\begin{array}{l}10.57 \\
14.63 \\
16.38\end{array}$ & $\begin{array}{r}5,200 \\
10,500 \\
13,800\end{array}$ & 12.09 \\
\hline 6 & $\begin{array}{l}\text { Little Sandy Creek } \\
\text { at Kisatchie. }\end{array}$ & 21.4 & $1949-68$ & 1953 & 10 & $\begin{array}{l}15.36 \\
12.55\end{array}$ & $\begin{array}{l}5,880 \\
4,140\end{array}$ & 10 \\
\hline 7 & Horsepen Creek & 5.27 & $1949-68$ & 1953 & & 11.88 & 1,910 & 16 \\
\hline 8 & $\begin{array}{l}\text { Little River near } \\
\text { Rochelle. }\end{array}$ & 1,880 & $1958-68$ & 1966 & 11 & $\begin{array}{l}39.22 \\
39.88\end{array}$ & $\begin{array}{l}44,700 \\
45,300\end{array}$ & 8 \\
\hline 9 & $\begin{array}{l}\text { Bayou Funny } \\
\text { Louis near } \\
\text { Trout. }\end{array}$ & 92 & $\begin{array}{l}1939-68 \\
\cdots\end{array}$ & 1953 & 8 & $\begin{array}{l}23.26 \\
18.50\end{array}$ & $\begin{array}{r}32,700 \\
6,310\end{array}$ & 8 \\
\hline 10 & $\begin{array}{l}\text { Big Creek at } \\
\text { Pollock. }\end{array}$ & 51 & $1942-68$ & $\begin{array}{c}1953 \\
\cdots\end{array}$ & 9 & $\begin{array}{l}18.03 \\
13.68\end{array}$ & $\begin{array}{r}23,500 \\
6,080 \\
\end{array}$ & 26 \\
\hline \multicolumn{9}{|c|}{ Sabine River basin } \\
\hline 11 & $\begin{array}{l}\text { Harpoon Bayou } \\
\text { at Many. }\end{array}$ & ${ }^{3} 41.6$ & $1951-68$ & 1961 & 8 & $\begin{array}{l}14.13 \\
16.61\end{array}$ & $\begin{array}{r}7,850 \\
23,800\end{array}$ & 12.43 \\
\hline 12 & Blackwell Creek & 3.16 & $1960-68$ & 1961 & & 13.20 & $\begin{array}{l}865 \\
812\end{array}$ & $6^{-\cdot}$ \\
\hline 13 & $\begin{array}{l}\text { Bayou Toro near } \\
\text { Florien. }\end{array}$ & 78.6 & $1950-68$ & $\overline{1953}$ & $8^{8}$ & $\begin{array}{l}12.33 \\
50.54 \\
50.95\end{array}$ & $\begin{array}{r}18,500 \\
\left({ }^{2}\right)\end{array}$ & $\cdots$ \\
\hline 14 & $\begin{array}{l}\text { Bayou Toro } \\
\text { near Toro. }\end{array}$ & 148 & $1956-68$ & 1961 & $8^{-}$ & $\begin{array}{l}24.24 \\
25.73\end{array}$ & $\begin{array}{l}20,500 \\
31,200\end{array}$ & 11.39 \\
\hline
\end{tabular}

1 Ratio of peak discharge to 50 -year flood.

2 Not determined.

${ }^{3}$ During floods only. Drainage area $22.7 \mathrm{sq} \mathrm{mi}$ during low water and medium flows.

\section{FLOODS OF APRIL 16 IN EASTERN MAUI, HAWAII}

The weather over the Hawaiian Islands prior to April 14 was relatively dry. On April 14, a surface low-pressure trough, east of Hawaii, moved slowly westward towards the islands. This trough brought cloudy conditions and frequent showers to the State. On the morning of the 15th, a high-pressure ridge at upper levels appeared to be almost stationary over the islands. Another upper level low-pressure area was to the west of the island chain and was moving slowly eastward. Considerable high- and middlelevel clouds were present. The displacement of the high-pressure ridge by the trough, together with the surface low, brought the heavy rains over eastern Maui on April 15. The heavy rain caused large amounts of damage to the Hana district (fig. 6). Other parts of the State also received some heavy rainfall, but no serious damage resulted. Hana Airport measured 17.57 inches of rain for the 24-hour period ending at 8:00 a.m. on April 16. Hana station measured 16.0 inches for the same period. Of this total, 15.96 inches fell between 4:00 p.m. on April 15 and 7:00 a.m. on

3 Modifled from U.S. Corps of Engineers (1968b). 
April 16. Slightly more than 1.80 inches fell within 15 minutes from 9:30 p.m. This intensity has a recurrence interval of over 100 years based on the rainfall-frequency atlas of the Hawaiian Islands (U.S. Weather Bureau, 1962). Kipahulu measured 12.53 inches in 12 hours, and Kaupo Ranch House, another station in southeastern Maui, registered 10.85 inches in 12 hours from 8:00 p.m. on April 15. The greatest intensity registered was 2.3 inches for a 30-minute period. Stations in other parts of the island measured little or no rain for the same period.

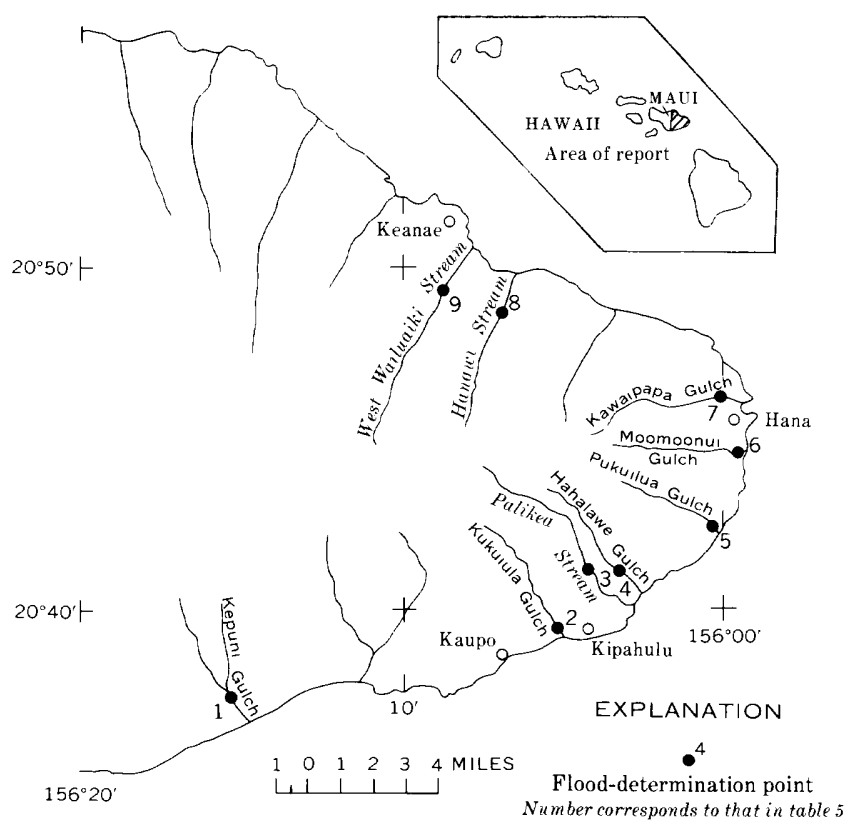

FigURE 6.-Flood area; location of flood-determination points, floods of April 16 in eastern Maui, Hawaii.

The highway from Hana to Kipahulu was impassable due to water, mud, and debris. Sections of the road shoulders and pavement were washed out. Small landslides partially blocked the narrow paved road. Board of Water Supply pipelines were also damaged, temporarily disrupting water service in some communities. Damage in the area from Kipahulu to Kaupo was very extensive. About 6 miles of the narrow, unpaved road was closed to traffic. This highway, benched into the steep mountain slopes, is the only road serving several small villages on the eastern coast 
of the island. Four timber bridges and three culverts were destroyed. Bridge approaches at four other crossings were washed out, severely damaging one bridge and causing minor damage to the others. Erosion at fords was extensive. Only one timber bridge remained undamaged from the high flows. Numerous landslides, with a total estimated volume of 5,000 cubic yards, covered the highway at several places and knocked down telephone lines. Roadbeds were also eroded by the hillside runoff. River crossings were widened considerably when bridge approaches and abutments were washed out. Several meandering stream channels were straightened; in other basins, new channels were formed in lower reaches when boulders tumbling down from upstream filled the existing streambeds. Streams in this area are normally dry or carry only trickles of water most of the year. Total damage between Kipahulu and Kaupo was about $\$ 230,000$.

The small community of Kaupo was completely isolated from Hana and Wailuku. Silt, debris, and large boulders blocked two road crossings of streams west of Kaupo.

Record-high discharges occurred at two crest-stage gages in Hana. Moomoonui Gulch (No. 6, table 5) in operation since 1963, had a peak discharge of 2,2,0 cfs. Kawaipapa Gulch (No. 7, table $5)$ with only 3 years of record, had a peak discharge of 14,000 cfs. Gaging stations at Palikea Stream (No. 3, table 5) and Hahalawe Gulch (No. 4, table 5) near Kipahulu had peak flows of 5,320 and $988 \mathrm{cfs}$, respectively, during the storm.

TABLE 5.-Flood stages and discharges, April 16 in eastern Maui, Hawaii

\begin{tabular}{|c|c|c|c|c|c|c|c|}
\hline \multirow{3}{*}{ No. } & \multirow{3}{*}{$\begin{array}{l}\text { Stream and place } \\
\text { of determination }\end{array}$} & \multirow{3}{*}{$\begin{array}{l}\text { Drainage } \\
\text { area } \\
\text { (sq mi) }\end{array}$} & \multicolumn{5}{|c|}{ Maximum floods } \\
\hline & & & \multicolumn{2}{|c|}{$\begin{array}{l}\text { Known before } \\
\text { April } 1968\end{array}$} & \multirow{2}{*}{$\begin{array}{l}\text { April } \\
1968\end{array}$} & \multirow{2}{*}{$\begin{array}{l}\text { Gage } \\
\text { height } \\
\text { (feet) }\end{array}$} & \multirow{2}{*}{$\frac{\text { Discharge }}{\text { Cfs }}$} \\
\hline & & & Period & Year & & & \\
\hline 1 & $\begin{array}{l}\text { Kepuni Gulch near } \\
\text { Kahikinui }\end{array}$ & 1.91 & $1963-58$ & 1967 & 16 & $\begin{array}{l}8.23 \\
4.52\end{array}$ & $\begin{array}{r}1,000 \\
195\end{array}$ \\
\hline 2 & $\begin{array}{l}\text { Kukuiula Gulch } \\
\text { near Kipahulu. }\end{array}$ & .76 & $1964-68$ & 1965 & 16 & $\begin{array}{r}5.11 \\
16.85\end{array}$ & $\begin{array}{r}1,560 \\
2,760\end{array}$ \\
\hline 3 & $\begin{array}{l}\text { Palikea Stream } \\
\text { below diversion } \\
\text { dam near } \\
\text { Kipahulu. }\end{array}$ & 6.29 & $\begin{array}{c}1927-29 \\
1931-68 . \\
\end{array}$ & 1965 & 16 & $\begin{array}{l}18.3 \\
9.69\end{array}$ & $\begin{array}{r}16,100 \\
5,320\end{array}$ \\
\hline 1 & $\begin{array}{c}\text { Hahalawe Gulch } \\
\text { near Kipahulu. }\end{array}$ & .43 & $\begin{array}{r}1927-37 \\
1939-68\end{array}$ & 1937 & $-\quad-$ & 15.74 & 3,560 \\
\hline 5 & $\begin{array}{c}\text { Pukuilua Gulch } \\
\text { near Hana. }\end{array}$ & .48 & $1963-68$ & 1965 & 16 & $\begin{array}{l}4.88 \\
9.3 \\
7.76\end{array}$ & $\begin{array}{l}988 \\
788 \\
594\end{array}$ \\
\hline 6 & $\begin{array}{l}\text { Moomoonui Gulch } \\
\text { at Hana. }\end{array}$ & .90 & $1963-68$ & $\overline{19} \overline{6} 5$ & 16 & $\begin{array}{l}14.46 \\
14.64\end{array}$ & $\begin{array}{l}2,070 \\
2,200\end{array}$ \\
\hline 7 & $\begin{array}{l}\text { Kawaipapa } \\
\text { Gulch at } \\
\text { Hana. }\end{array}$ & 5.83 & $1965-68$ & 1965 & 16 & $\begin{array}{r}9.59 \\
10.36\end{array}$ & $\begin{array}{l}10,200 \\
14,000\end{array}$ \\
\hline 8 & $\begin{array}{l}\text { Hanawi Stream } \\
\text { near Nahiko. }\end{array}$ & 3.49 & $\begin{array}{l}1914-16 \\
\quad 1922-68 .\end{array}$ & 1916 & $-x_{10}$ & 11.6 & 5,570 \\
\hline 9 & $\begin{array}{l}\text { West Wailuaiki } \\
\text { Stream near }\end{array}$ & 3.66 & $\begin{array}{c}1914-1 \overline{7}, \\
1922-68 .\end{array}$ & 1923 & 16 & $\begin{array}{r}8.15 \\
13.50\end{array}$ & $\begin{array}{l}2,660 \\
6,960\end{array}$ \\
\hline & Keanae. & & $\begin{array}{l}1922-60 \\
-\cdots\end{array}$ & - - - & 16 & 8.07 & 2.490 \\
\hline
\end{tabular}


Damage in the flood area was estimated at $\$ 293,000$. The torrential rains and resulting runoff destroyed a culvert at Honomaele Gulch on Hana Highway, which was closed to traffic until a temporary bypass road was constructed. Flows over the rolling pastureland flooded the roadways in Hana and severely damaged a 200-foot section of a drainage ditch, whose lining, bedding, and sections of earth banks were washed downstream into the ocean. Road shoulders, retaining walls and pavement on a 50 -foot section of the lower Hana Road near Holoinawawae Stream were also damaged. Damage was about $\$ 35,000$. The Hotel Hana Maui had about $\$ 5,000$ damage to carpets, furniture, and supplies when water rushed over the highway into the building. Roads, fences, waterlines, and feed storage areas on Hana Ranch were also damaged. Other damage in Hana consisted of toppled retaining walls and debris left at private residences.

\section{FLOODS OF MAY IN SOUTH ARKANSAS}

Heavy rains fell on south Arkansas during the period May $7-18$, with the greatest amount falling on the area extending from Little Rock to the southwest corner of the State (fig. 7).

Runoff from the storm of May 9-10 was not especially heavy, but wetting of the soil at this time induced high rates of runoff from the second storm of May 13. Floods during May 9-10 were greatest in the upper reaches of the Saline River (tributary to Ouachita River), on the Little Missouri River and tributaries, and on Caney Creek (tributary to the Red River). Rainfall of up to 10 inches in 24 hours caused streams to rise rapidly, but the peaks were of short duration except those on larger and slowmoving streams.

Floods from the storm of May 13 were much more severe and widespread. The greatest flooding during the period May 16-17 was on Smackover Creek and other smaller streams in the southern part of the flood area.

Flood damage was estimated at $\$ 18.3$ million. No lives were lost. Many residential and industrial areas were flooded. Millions of acres of timberland, farmland, and pastures were inundated, eroded, and silted over. Many highway bridges were damaged.

A report by Gilstrap (1971) describes the flood in detail. The report discusses the storms, the floods, and the flood damage. Flood data in the report include peak stages and discharges at 95 sites, flood profiles of three streams, recurrence intervals of peak discharges, rainfall-runoff relations, and reservoir contents. 


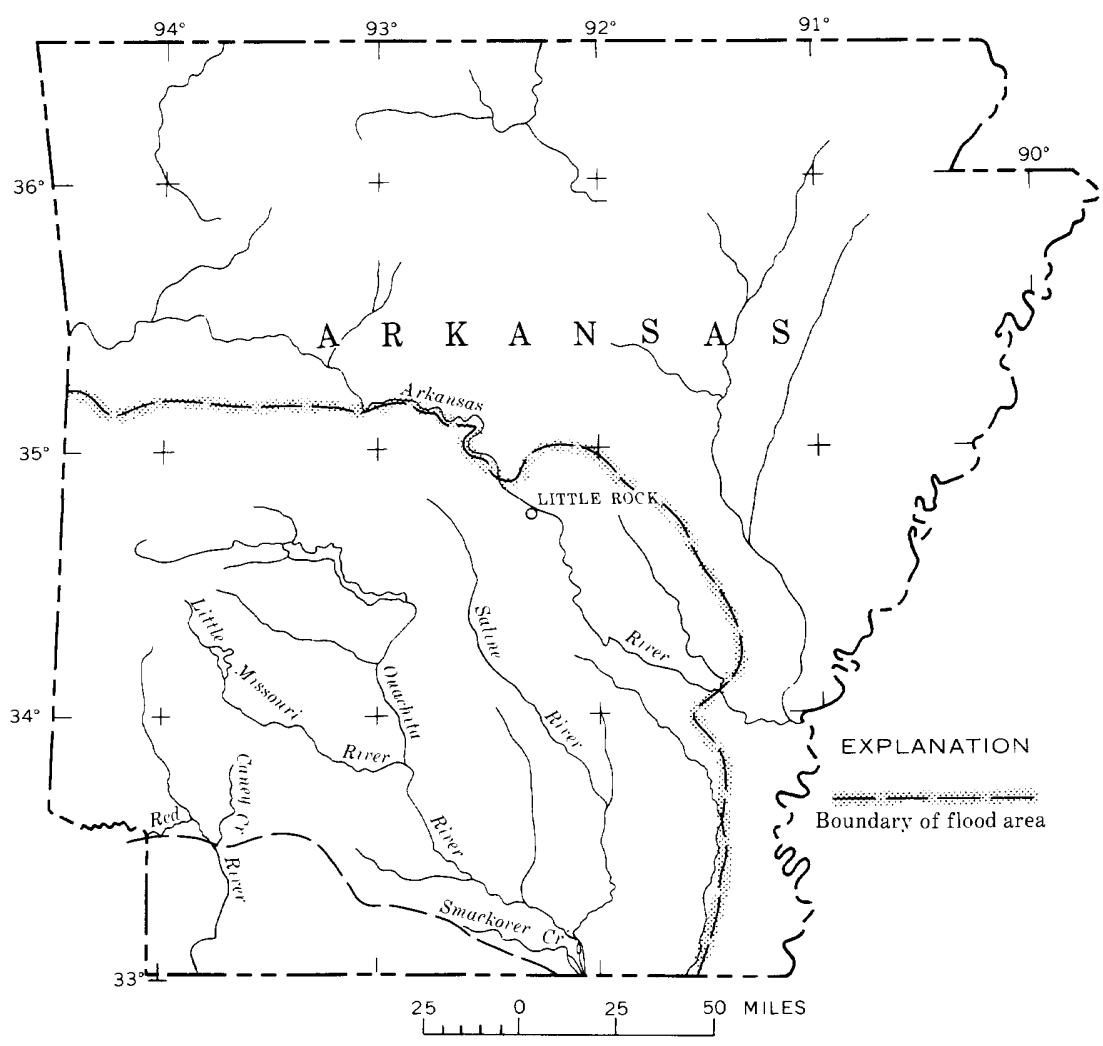

Figure 7.-Flood area, floods of May in south Arkansas.

\section{FLOODS OF MAY 24-27 IN SOUTHERN INDIANA}

Severe flooding occurred in southeastern and west-central Indiana as the result of heavy rainfall during May 22-25. Precipitation ranged from 3 to 7 inches and generally progressed from west to east across the center of Indiana. An isohyetal map of Indiana (fig. 8) was compiled from U.S. Weather Bureau data for the period May 22-25.

Maximum stages during periods of record ranging from 3 to 40 years in length were equaled or exceeded at three gaging stations (table 6 ). The recurrence interval of the floods equaled or exceeded 50 years at five of these gaging stations, 20 years at six stations, and 10 years at 17 stations.

Flooding in urban areas of Johnson, Shelby, and Bartholomew Counties caused widespread damage to homes and small businesses. Damage in rural areas of Jackson and Jennings Counties was estimated at $\$ 750,000$. 


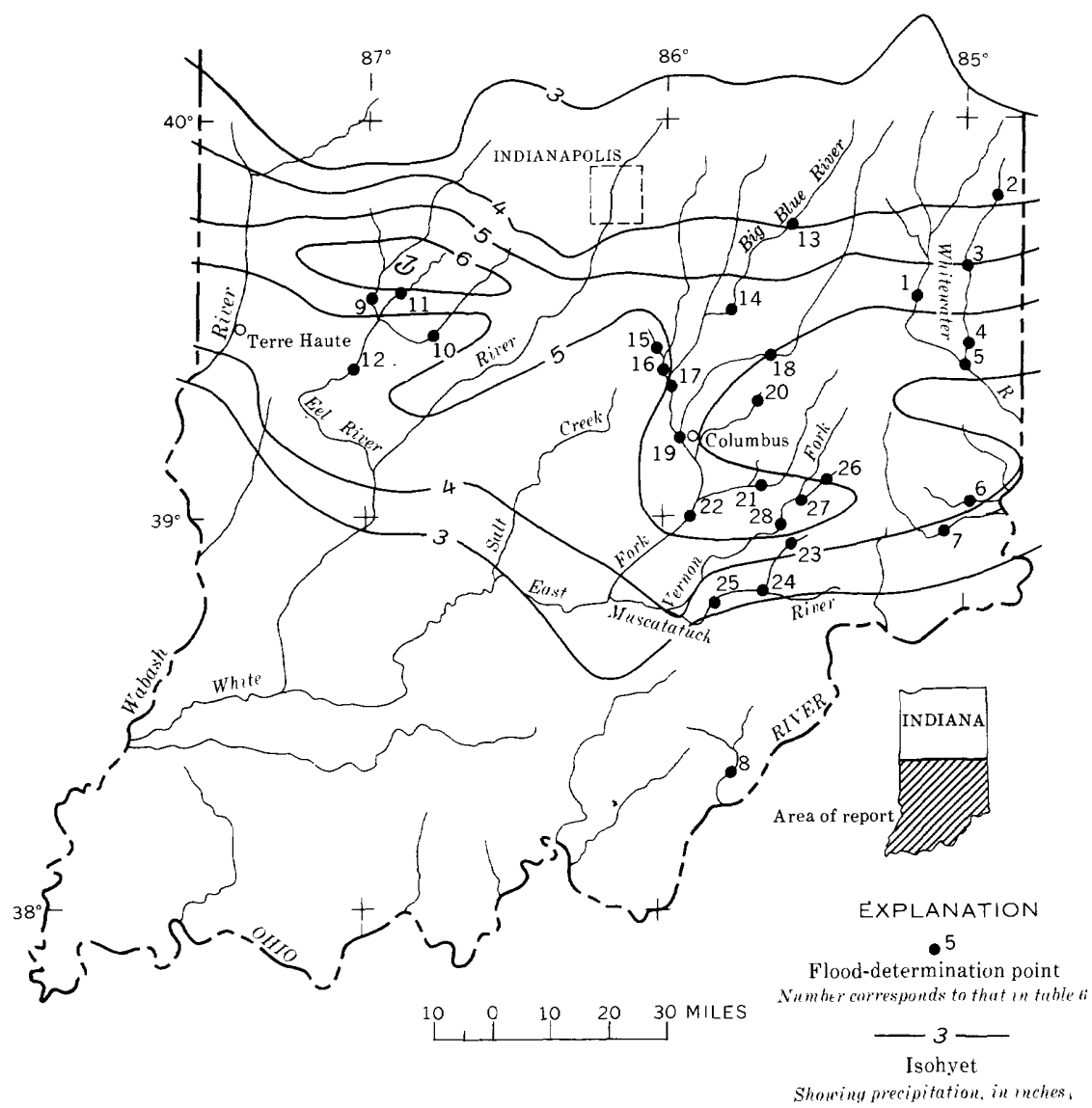

FIGURE 8.-Flood area; location of flood-determination points and isohyets for May 22-25, floods of May 24-27 in southern Indiana.

TABLE 6.-Flood stages and discharges, May 24-27 in southern Indiana

\begin{tabular}{|c|c|c|c|c|c|c|c|c|}
\hline \multirow{4}{*}{ No. } & \multirow{4}{*}{$\begin{array}{l}\text { Stream and place } \\
\text { of determination }\end{array}$} & \multirow{4}{*}{$\begin{array}{l}\text { Drainage } \\
\text { area } \\
\text { (sq mi) }\end{array}$} & \multicolumn{6}{|c|}{ Maximum floods } \\
\hline & & & \multirow{2}{*}{\multicolumn{2}{|c|}{$\begin{array}{l}\text { Known before } \\
\text { May } 1968\end{array}$}} & \multirow{3}{*}{$\begin{array}{l}\text { May } \\
1968\end{array}$} & \multirow{3}{*}{$\begin{array}{c}\text { Gage } \\
\text { height } \\
\text { (feet) }\end{array}$} & \multicolumn{2}{|c|}{ Discharge } \\
\hline & & & & & & & & \\
\hline & & & Period & Year & & & Cfs & $\begin{array}{c}\text { interval } \\
\text { (years) }\end{array}$ \\
\hline \multicolumn{9}{|c|}{ Miami River basin } \\
\hline 1 & $\begin{array}{l}\text { Whitewater River } \\
\text { near Alpine }\end{array}$ & 529 & $\begin{array}{l}1929-68 \\
-\end{array}$ & 1937 & 24 & $\begin{array}{l}16.61 \\
15.81\end{array}$ & $\begin{array}{l}37,100 \\
27,600\end{array}$ & 14 \\
\hline 2 & $\begin{array}{l}\text { East Fork White- } \\
\text { water River at } \\
\text { Richmond. }\end{array}$ & 121 & $\begin{array}{l}1913 \\
1949-68\end{array}$ & $\begin{array}{l}1913 \\
1950 \\
1959\end{array}$ & 24 & $\begin{array}{l}15.0 \\
12.49 \\
10.06\end{array}$ & $\begin{array}{c}\text { (1) } \\
14,100 \\
7,500\end{array}$ & $\overline{-}^{-}$ \\
\hline 3 & $\begin{array}{l}\text { East Fork White- } \\
\text { water River at } \\
\text { Abington. }\end{array}$ & 198 & $1966-68$ & 1967 & 24 & $\begin{array}{l}13.54 \\
14.05\end{array}$ & $\begin{array}{l}6,300 \\
9,980\end{array}$ & $\overline{6}^{-\cdots}$ \\
\hline 4 & $\begin{array}{l}\text { East Fork White- } \\
\text { water River at } \\
\text { Brookville. }\end{array}$ & 380 & $1954-68$ & 1959 & 24 & $\begin{array}{r}216.50 \\
17.35\end{array}$ & $\begin{array}{l}36,100 \\
31,600\end{array}$ & 18 \\
\hline
\end{tabular}

See footnotes at end of table. 
TABLE 6-Flood stages and discharges, May 24-27 in southern IndianaContinued

\begin{tabular}{|c|c|c|c|c|c|c|c|c|}
\hline \multirow{4}{*}{ No. } & \multirow{4}{*}{$\begin{array}{l}\text { Stream and place } \\
\text { of determination }\end{array}$} & \multirow{4}{*}{$\begin{array}{l}\text { Drainage } \\
\text { area } \\
\text { (sq mi) }\end{array}$} & \multicolumn{6}{|c|}{ Maximum floods } \\
\hline & & & \multirow{2}{*}{\multicolumn{2}{|c|}{$\begin{array}{l}\text { Known before } \\
\text { May } 1968\end{array}$}} & \multirow{3}{*}{$\begin{array}{l}\text { May } \\
1968\end{array}$} & \multirow{3}{*}{$\begin{array}{c}\text { Gage } \\
\text { height } \\
\text { (feet) }\end{array}$} & \multicolumn{2}{|c|}{ Discharge } \\
\hline & & & & & & & \multirow[b]{2}{*}{ Cfs } & \multirow{2}{*}{$\begin{array}{c}\text { Recur- } \\
\text { rence } \\
\text { interval } \\
\text { (years) }\end{array}$} \\
\hline & & & Period & Year & & & & \\
\hline \multicolumn{9}{|c|}{ Miami River basin-Conitnued } \\
\hline \multirow[t]{2}{*}{5} & $\begin{array}{l}\text { Whitewater River } \\
\text { at Brookville. }\end{array}$ & 1,224 & $\begin{array}{l}1913 \\
1916-17\end{array}$ & $\begin{array}{l}1913 \\
1959\end{array}$ & --- & $\begin{array}{l}39.0 \\
27.78\end{array}$ & $\stackrel{(1)}{81,800}$ & $\cdots$ \\
\hline & & & $1924-00$ & $\ldots$ & 24 & 23.55 & 57,800 & 7 \\
\hline \multicolumn{9}{|c|}{ Hogan Creek basin } \\
\hline 6 & $\begin{array}{c}\text { South Hogan } \\
\text { Creek near } \\
\text { Dillsboro. }\end{array}$ & 38.2 & $\begin{array}{l}1959 \\
1962-68\end{array}$ & $\begin{array}{l}1959 \\
1963\end{array}$ & $\therefore$ & $\begin{array}{l}14.00 \\
10.82\end{array}$ & $\begin{array}{r}16,300 \\
8,630\end{array}$ & 50 \\
\hline \multicolumn{9}{|c|}{ Laughery Creek basin } \\
\hline 7 & $\begin{array}{c}\text { Laughery Creek } \\
\text { near Farmers } \\
\text { Retreat. }\end{array}$ & 248 & $1941-68$ & 1959 & 24 & $\begin{array}{l}21.13 \\
17.06\end{array}$ & $\begin{array}{l}47,800 \\
28,700\end{array}$ & 16 \\
\hline \multicolumn{9}{|c|}{ Silver Creek basin } \\
\hline 8 & $\begin{array}{l}\text { Silver Creek } \\
\text { near Sellers- } \\
\text { burg. }\end{array}$ & 188 & $\begin{array}{l}1955-68 \\
-\ldots---\end{array}$ & 1959 & $27^{--}$ & $\begin{array}{l}30.89 \\
18.45\end{array}$ & $\begin{array}{r}19,600 \\
4,000\end{array}$ & -- \\
\hline \multicolumn{9}{|c|}{ Wabash River basin } \\
\hline 9 & $\begin{array}{c}\text { Big Walnut Creek } \\
\text { near Reelsville. }\end{array}$ & 326 & $1950-68$ & 1957 & 24 & $\begin{array}{l}18.63 \\
17.07\end{array}$ & $\begin{array}{l}27,400 \\
15,800\end{array}$ & 17 \\
\hline 10 & $\begin{array}{l}\text { Mill Creek near } \\
\text { Cataract. }\end{array}$ & 245 & $1950-68$ & 1960 & $\overline{24}$ & $\begin{array}{l}22.58 \\
21.54\end{array}$ & $\begin{array}{l}11,400 \\
10,200\end{array}$ & $8^{\cdots}$ \\
\hline 11 & $\begin{array}{l}\text { Deer Creek near } \\
\text { Putnamville. }\end{array}$ & 59.0 & $\begin{array}{c}1955-65 \\
1968\end{array}$ & 1963 & 24 & $\begin{array}{l}12.95 \\
15.18\end{array}$ & $\begin{array}{r}10,700 \\
8,900\end{array}$ & ${ }^{3} 1.5$ \\
\hline 12 & $\begin{array}{l}\text { Eel River at } \\
\text { Bowling Green. }\end{array}$ & 830 & $\begin{array}{l}1875 \\
1931-68\end{array}$ & $\begin{array}{l}1875 \\
1950\end{array}$ & 24 & $\begin{array}{l}30.0 \\
23.53 \\
21.52\end{array}$ & $\begin{array}{c}(1) \\
34,000 \\
22,100\end{array}$ & $6^{--}$ \\
\hline 13 & $\begin{array}{l}\text { Big Blue River at } \\
\text { Carthage }\end{array}$ & 184 & $1951-68$ & 1963 & $\overline{24}$ & $\begin{array}{r}14.62 \\
9.66\end{array}$ & $\begin{array}{r}12,900 \\
4,340\end{array}$ & $4^{\cdots+}$ \\
\hline 14 & $\begin{array}{l}\text { Big Blue River at } \\
\text { Shelbyville. }\end{array}$ & 421 & $\begin{array}{l}1913 \\
1944-68 \\
-\end{array}$ & $\begin{array}{l}1913 \\
1963\end{array}$ & 24 & $\begin{array}{l}20.2 \\
17.70 \\
16.10\end{array}$ & $\begin{array}{c}(1) \\
15,800 \\
11,500\end{array}$ & 3 \\
\hline 15 & $\begin{array}{l}\text { Youngs Creek } \\
\text { near Edinburg. }\end{array}$ & 107 & $1943-68$ & $\begin{array}{r}1952 \\
-\end{array}$ & 24 & $\begin{array}{l}13.4 \\
12.85\end{array}$ & $\begin{array}{r}10,700 \\
9,290\end{array}$ & 3.1 \\
\hline 16 & $\begin{array}{l}\text { Sugar Creek near } \\
\text { Edinburg. }\end{array}$ & 474 & $1943-68$ & 1956 & 24 & $\begin{array}{l}18.38 \\
16.98\end{array}$ & $\begin{array}{l}27,600 \\
19,900\end{array}$ & 17 \\
\hline 17 & $\begin{array}{l}\text { Driftwood River } \\
\text { near Edinburg. }\end{array}$ & 1,060 & $\begin{array}{l}1913 \\
1941-68\end{array}$ & $\begin{array}{l}1913 \\
1963\end{array}$ & 24 & $\begin{array}{l}20.3 \\
16.97 \\
16.61\end{array}$ & $\begin{array}{c}(1) \\
40,500 \\
34,600\end{array}$ & 14 \\
\hline 18 & $\begin{array}{l}\text { Flatrock River at } \\
\text { St. Paul. }\end{array}$ & 303 & $\begin{array}{l}1913 \\
1931-68\end{array}$ & $\begin{array}{l}1913 \\
1949 \\
1963\end{array}$ & 24 & $\begin{array}{l}20.5 \\
12.17 \\
12.37\end{array}$ & $\begin{array}{c}\stackrel{(1)}{1} \\
18,500 \\
17,600\end{array}$ & $\overline{13}^{\cdots}$ \\
\hline 19 & $\begin{array}{l}\text { East Fork } \\
\text { White River } \\
\text { at Columbus. }\end{array}$ & 1,707 & $1948-68$ & 1963 & 25 & $\begin{array}{l}16.23 \\
14.99\end{array}$ & $\begin{array}{l}52,300 \\
44,700\end{array}$ & 10 \\
\hline 20 & $\begin{array}{l}\text { Clifty Creek at } \\
\text { Hartsville. }\end{array}$ & 91.4 & $\begin{array}{l}1913 \\
1948-68\end{array}$ & $\begin{array}{l}1913 \\
1959\end{array}$ & 24 & $\begin{array}{l}25.1 \\
14.29 \\
11.79\end{array}$ & $\begin{array}{r}\left({ }^{1}\right) \\
11,300 \\
6,840\end{array}$ & 10 \\
\hline 21 & $\begin{array}{l}\text { Sand Creek near } \\
\text { Brewersville. }\end{array}$ & 155 & $1948-68$ & 1959 & 24 & $\begin{array}{l}21.70 \\
20.08\end{array}$ & $\begin{array}{l}19,900 \\
15,200\end{array}$ & 13 \\
\hline 22 & $\begin{array}{l}\text { East Fork White } \\
\text { River at } \\
\text { Seymour. }\end{array}$ & 2,341 & $\begin{array}{l}1913 \\
1928-68 \\
-\end{array}$ & $\begin{array}{l}1913 \\
1949\end{array}$ & 24 & $\begin{array}{l}21.0 \\
19.67 \\
18.93\end{array}$ & $\begin{array}{r}120,000 \\
78,500 \\
60,200\end{array}$ & $\mathrm{is}^{-}$ \\
\hline 23 & $\begin{array}{l}\text { Graham Creek near } \\
\text { Vernon. }\end{array}$ & 77.2 & $1954-68$ & 1960 & 24 & $\begin{array}{l}21.37 \\
19.98\end{array}$ & $\begin{array}{l}18,600 \\
16.300\end{array}$ & $46^{-}$ \\
\hline
\end{tabular}

See footnotes at end of table. 
TABLE 6-Flood stages and discharges, May 24-27 in southern IndianaContinued

\begin{tabular}{|c|c|c|c|c|c|c|c|c|}
\hline \multirow{4}{*}{ No. } & \multirow{4}{*}{$\begin{array}{l}\text { Stream and place } \\
\text { of determination }\end{array}$} & \multirow{4}{*}{$\begin{array}{c}\text { Drainage } \\
\text { area } \\
\text { (sq mi) }\end{array}$} & \multicolumn{6}{|c|}{ Maximum floods } \\
\hline & & & \multirow{2}{*}{\multicolumn{2}{|c|}{$\begin{array}{l}\text { Known before } \\
\text { May } 1968\end{array}$}} & \multirow{3}{*}{$\begin{array}{l}\text { May } \\
1968\end{array}$} & \multirow{3}{*}{$\begin{array}{c}\text { Gage } \\
\text { height } \\
\text { (feet) }\end{array}$} & \multicolumn{2}{|c|}{ Discharge } \\
\hline & & & & & & & & Recur- \\
\hline & & & Period & Year & & & Cfs & $\begin{array}{l}\text { interva } \\
\text { (years) }\end{array}$ \\
\hline \multicolumn{9}{|c|}{ Wabash River basin-Continued } \\
\hline 24 & $\begin{array}{l}\text { Muscatatuck } \\
\text { River near } \\
\text { Deputy. }\end{array}$ & 293 & $1948-68$ & $\begin{array}{l}1959 \\
-\end{array}$ & 24 & $\begin{array}{l}33.1 \\
27.05\end{array}$ & $\begin{array}{l}52,200 \\
26,300\end{array}$ & 7 \\
\hline 25 & $\begin{array}{l}\text { Muscatatuck River } \\
\text { near Austin. }\end{array}$ & 359 & $1933-68$ & 1959 & 25 & $\begin{array}{l}29.20 \\
25.68\end{array}$ & $\begin{array}{l}53,900 \\
22,200\end{array}$ & 5 \\
\hline 26 & $\begin{array}{l}\text { Brush Creek } \\
\text { near Nebraska. }\end{array}$ & 11.4 & $1955-68$ & $\begin{array}{l}1962 \\
--\end{array}$ & 24 & $\begin{array}{l}10.90 \\
11.40\end{array}$ & $\begin{array}{l}3,460 \\
3,440\end{array}$ & $\left({ }^{1}\right)$ \\
\hline 27 & $\begin{array}{l}\text { Vernon Fork } \\
\text { near Butlerville. }\end{array}$ & 85.9 & $1942-68$ & 1959 & 24 & $\begin{array}{l}25.41 \\
23.20\end{array}$ & $\begin{array}{l}26,200 \\
19,500\end{array}$ & 31.1 \\
\hline 28 & $\begin{array}{l}\text { Vernon Fork at } \\
\text { Vernon. }\end{array}$ & 198 & $\begin{array}{c}1940-68 \\
-\end{array}$ & 1959 & 24 & $\begin{array}{l}32.83 \\
29.46\end{array}$ & $\begin{array}{l}56,800 \\
40,600\end{array}$ & 31.3 \\
\hline
\end{tabular}

1 Not determined.

2 At different site and datum.

${ }^{3}$ Ratio of peak discharge to 50 -year flood.

\section{FLOODS OF MAY 24-28 IN SOUTHERN OHIO}

By E. E. WebBer

Precipitation from 3 inches to more than 5 inches during the night of May 23-24, followed by 2 to 4 inches of additional precipitation on the night of May 26-27, produced severe flooding in southern Ohio during the period May 24-28 (fig. 9). The ground in the flood area had been saturated by antecedent precipitation of 3 to 5 inches during the period May 8-22. Throughout most of the area, the highest peak discharge occurred on May 24 or 25 , followed by a lower secondary peak resulting from the storm of May 26-27. On Raccoon Creek at Adamsville (No. 5, table 7) and on Ohio Brush Creek near West Union (No. 16, table 7), the highest peak discharges occurred after the second storm period.

The highest runoff and the most severe flooding occurred in the Hocking River, Raccoon Creek, Salt Creek, Paint Creek, and Little Miami River basins. (See table 7.) The highest stage since 1907 was recorded on the Hocking River at Athens (No. 3, table 7 ), and the record-breaking discharge of $22,500 \mathrm{cfs}$ on Raccoon Creek at Adamsville was estimated to be 1.3 times that of a 50year flood. Four hundred residents of Laurelville, in the Salt Creek basin, were forced to evacuate their homes. Near the proposed Salt Creek dam (No. 15, table 7), an unofficial stage record for the 81-year period 1888-1968 was exceeded by this flood. Extreme flooding occurred in the upper reaches of Paint Creek, where the runoff resulting from precipitation cells of 5 to 7.5 
inches caused considerable loss of livestock and wildlife. The midportion of Little River basin suffered the most damage in that basin; the peak of $25,400 \mathrm{cfs}$ at Todd Fork near Roachester (No. 21 , table 7) was estimated to be 1.4 times that of the 50-year flood.

Locations of the 24 flood-determination points, and isohyets for May 23-24, 1968, are shown in figure 9. A summary of peak stages, discharges, and related data at the 24 sites is listed in table 7. Composite flood-frequency curves applicable to Ohio (Cross and Webber, 1959, and Speer and Gamble, 1965) were used to estimate the recurrence intervals shown in table 7 . The greatest urban damage was at Laurelville and Athens, and throughout the storm area many roads and bridges were destroyed. Two lives were lost. Total damage in the 31 counties affected has been estimated by the U.S. Weather Bureau at $\$ 9$ million.

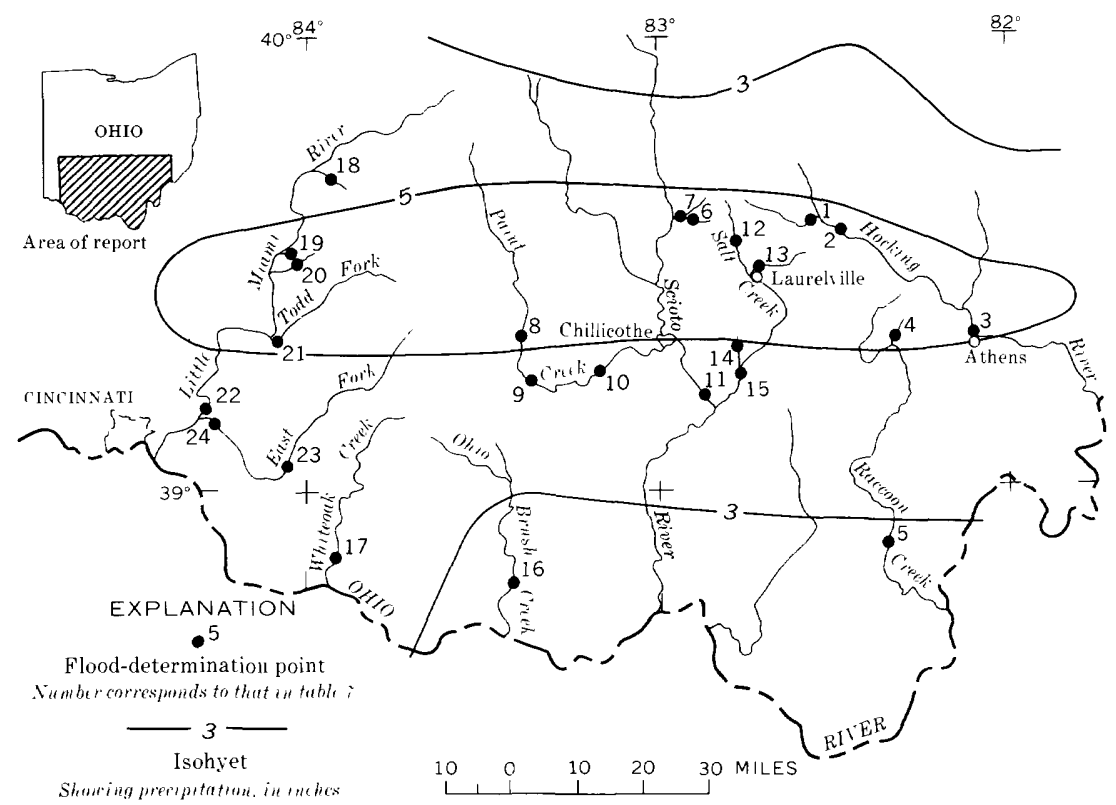

Figrese 9.-Flood area; location of flood-determination points and isohyets for May 23-24, floods of May 24-28 in southern Ohio. 
TABLE 7.-Flood stages and discharges, May 24-28 in southern Ohio

\begin{tabular}{|c|c|c|c|c|c|c|c|c|}
\hline \multirow{4}{*}{ No. } & \multirow{4}{*}{$\begin{array}{l}\text { Stream and place } \\
\text { of determination }\end{array}$} & \multirow{4}{*}{$\begin{array}{c}\text { Drainage } \\
\text { area } \\
\text { (sq mi) }\end{array}$} & \multicolumn{6}{|c|}{ Maximum floods } \\
\hline & & & \multirow{2}{*}{\multicolumn{2}{|c|}{$\begin{array}{l}\text { Known before } \\
\text { May } 1968\end{array}$}} & \multirow{3}{*}{$\begin{array}{l}\text { May } \\
1968\end{array}$} & \multirow{3}{*}{$\begin{array}{l}\text { Gage } \\
\text { height } \\
\text { (feet) }\end{array}$} & \multicolumn{2}{|c|}{ Discharge } \\
\hline & & & & & & & \multirow[b]{2}{*}{ Cfs } & \multirow{2}{*}{$\begin{array}{c}\text { Recur- } \\
\text { rence } \\
\text { interval } \\
\text { (years) }\end{array}$} \\
\hline & & & Period & Year & & & & \\
\hline \multicolumn{9}{|c|}{ Hocking River basin } \\
\hline 1 & $\begin{array}{l}\text { Clear Creek near } \\
\text { Rockbridge. }\end{array}$ & 89.0 & $1940-68$ & 1948 & 24 & $\begin{array}{l}17.68 \\
15.76\end{array}$ & $\begin{array}{l}16,000 \\
11,000\end{array}$ & 11.1 \\
\hline 2 & $\begin{array}{l}\text { Hocking River at } \\
\text { Enterprise. }\end{array}$ & 459 & $1907-68$ & 1907 & 24 & $\begin{array}{l}22.0 \\
18.31\end{array}$ & $\begin{array}{l}36,000 \\
16,600\end{array}$ & $\overline{15}$ \\
\hline 3 & $\begin{array}{l}\text { Hocking River at } \\
\text { Athens. }\end{array}$ & 943 & $\begin{array}{l}1907-68 \\
-\end{array}$ & 1907 & $\mathbf{2 5}$ & $\begin{array}{l}27 . \\
24.65\end{array}$ & $\begin{array}{l}50,000 \\
32,400\end{array}$ & $2 \overline{7}$ \\
\hline \multicolumn{9}{|c|}{ Raccoon Creek basin } \\
\hline 4 & $\begin{array}{l}\text { Sandy Run near } \\
\text { Lake Hope. }\end{array}$ & 4.99 & $1958-68$ & 1958 & 24 & $\begin{array}{l}8.41 \\
8.32\end{array}$ & $\begin{array}{l}3,770 \\
2,440\end{array}$ & $\mathbf{5}$ \\
\hline 5 & $\begin{array}{l}\text { Raccoon Creek at } \\
\text { Adamsville. }\end{array}$ & 585 & $1915-68$ & $\begin{array}{l}1937 \\
-\ldots\end{array}$ & $\overline{28}$ & $\begin{array}{l}25.2 \\
28.69\end{array}$ & $\begin{array}{l}16,000 \\
22,500\end{array}$ & $1 \tilde{1.3}$ \\
\hline \multicolumn{9}{|c|}{ Scioto River basin } \\
\hline 6 & $\begin{array}{l}\text { Hominy Creek at } \\
\text { Circleville. }\end{array}$ & 5.66 & $1947-68$ & 1947 & 24 & $\begin{array}{l}10.16 \\
11.72\end{array}$ & $\begin{array}{l}2,100 \\
3,820\end{array}$ & 12.0 \\
\hline 7 & $\begin{array}{l}\text { Hargus Creek at } \\
\text { Circleville. }\end{array}$ & 19.6 & $\ldots \ldots$ & .. & 24 & $\ldots$ & 4,390 & $\left({ }^{2}\right)$ \\
\hline 8 & $\begin{array}{l}\text { Paint Creek near } \\
\text { Greenfield. }\end{array}$ & 249 & $\begin{array}{c}1927-35 \\
1940-56 \\
1963-68\end{array}$ & 1964 &.-- & 18.3 & 16,000 & $\ldots$ \\
\hline & & & . . . . & $\ldots$ & 24 & 14.28 & 21,700 & 11.2 \\
\hline 9 & $\begin{array}{l}\text { Paint Creek below } \\
\text { Paint Creek } \\
\text { Dam near } \\
\text { Bainbridge. }\end{array}$ & 570 & $1963-68$ & 1964 & 24 & $\begin{array}{l}327.3 \\
66.78\end{array}$ & $\begin{array}{l}45,000 \\
32,800\end{array}$ & $\left({ }^{2}\right)$ \\
\hline 10 & $\begin{array}{l}\text { Paint Creek near } \\
\text { Bourneville. }\end{array}$ & 80.7 & $\begin{array}{c}1922-36 \\
1938-68\end{array}$ & 1964 & -- & 20.50 & 56,900 & -... \\
\hline 11 & $\begin{array}{l}\text { Scioto River } \\
\text { at Higby. }\end{array}$ & 5,131 & $\begin{array}{l}1913-68 \\
1931-68 \\
\ldots \ldots\end{array}$ & $\begin{array}{l}1913 \\
1937 \\
-\end{array}$ & $\begin{array}{l}24 \\
-\cdots \\
25\end{array}$ & $\begin{array}{l}18.48 \\
31.6 \\
26.4 \\
22.41\end{array}$ & $\begin{array}{l}40,200 \\
(2) \\
177,000 \\
89,500\end{array}$ & $\begin{array}{l}10 \\
\cdots \\
\overline{5}^{-\cdots}\end{array}$ \\
\hline 12 & $\begin{array}{l}\text { Salt Creek at } \\
\text { Tarlton. }\end{array}$ & 11.5 & $1947-68$ & 1947 & 24 & $\begin{array}{l}{ }^{3} 6.4 \\
65.70\end{array}$ & $\begin{array}{l}\mathbf{2 , 7 8 0} \\
\mathbf{5 , 3 6 0}\end{array}$ & $1 \overline{1.9}$ \\
\hline 13 & $\begin{array}{c}\text { Laurel Run at } \\
\text { Laurelville. }\end{array}$ & 54.4 & 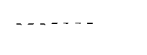 & $-\ldots$ & 24 & $-\ldots$ & 20,800 & $\left({ }^{2}\right)$ \\
\hline 14 & $\begin{array}{c}\text { Tar Hollow Creek } \\
\text { at Tar Hollow } \\
\text { State Park. }\end{array}$ & 1.35 & $1947-68$ & 1958 & 24 & $\begin{array}{l}5.62 \\
\mathbf{5 . 6 6}\end{array}$ & $\begin{array}{l}374 \\
957\end{array}$ & 12.5 \\
\hline 15 & $\begin{array}{l}\text { Salt Creek above } \\
\text { dam site near } \\
\text { Londonderry. }\end{array}$ & 268 & $1963-68$ & 1963 & $\mathbf{2 4}$ & $\begin{array}{l}22.7 \\
25.6\end{array}$ & $\begin{array}{l}31,600 \\
59,000\end{array}$ & 12.7 \\
\hline \multicolumn{9}{|c|}{ Ohio Brush Creek basin } \\
\hline 16 & $\begin{array}{l}\text { Ohio Brush Creek } \\
\text { near West }\end{array}$ & 387 & $\begin{array}{c}1927-35 \\
1941-68\end{array}$ & 1964 & $\cdots$ & 27.91 & 59,200 & $-\cdots$ \\
\hline & Union & & $1941-00$ & $-\cdots$ & 27 & 20.67 & 26,300 & 5 \\
\hline \multicolumn{9}{|c|}{ Whiteoak Creek basin } \\
\hline 17 & $\begin{array}{l}\text { Whiteoak Creek } \\
\text { near } \\
\text { Georgetown. }\end{array}$ & 222 & $\begin{array}{c}1924-35 \\
1940-68 . \\
\end{array}$ & $\begin{array}{l}1964 \\
1933 \\
-\end{array}$ & 24 & $\begin{array}{l}20.87 \\
10.63 \\
\end{array}$ & $\begin{array}{l}22,400 \\
12,400\end{array}$ & $4^{-\cdots}$ \\
\hline \multicolumn{9}{|c|}{ Little Miami River basin } \\
\hline 18 & $\begin{array}{l}\text { Shawnee Creek } \\
\text { at Xenia. }\end{array}$ & 4.21 & $1948-68$ & 1959 & 24 & $\begin{array}{l}16.02 \\
16.99\end{array}$ & $\begin{array}{r}855 \\
1,820\end{array}$ & 1.2 \\
\hline 19 & $\begin{array}{l}\text { Wayne Creek at } \\
\text { Waynesville. }\end{array}$ & 1.01 & $1966-68$ & 1966 & 24 & $\begin{array}{l}21.4 \\
26.37\end{array}$ & $\begin{array}{l}229 \\
640\end{array}$ & $\overline{(2)}^{--}$ \\
\hline 20 & $\begin{array}{c}\text { Caesar Creek at } \\
\text { Harveysburg. }\end{array}$ & 209 & $\begin{array}{l}1959-68 \\
-\ldots\end{array}$ & $\begin{array}{l}1959 \\
--\end{array}$ & $\overline{24}$ & $\begin{array}{l}20.5 \\
18.70\end{array}$ & $\begin{array}{l}26,000 \\
24,000\end{array}$ & $(2)$ \\
\hline
\end{tabular}

See footnotes at end of table. 
TABLE 7.-Flood stages and discharges, May 24-28 in southern OhioContinued

\begin{tabular}{|c|c|c|c|c|c|c|c|c|}
\hline \multirow{4}{*}{ No. } & \multirow{4}{*}{$\begin{array}{l}\text { Stream and place } \\
\text { of determination }\end{array}$} & \multirow{4}{*}{$\begin{array}{c}\text { Drainage } \\
\text { area } \\
\text { (sq mi) }\end{array}$} & \multicolumn{6}{|c|}{ Maximum floods } \\
\hline & & & \multirow{2}{*}{\multicolumn{2}{|c|}{$\begin{array}{l}\text { Known before } \\
\text { May } 1968\end{array}$}} & \multirow{3}{*}{$\begin{array}{l}\text { May } \\
1968\end{array}$} & \multirow{3}{*}{$\begin{array}{l}\text { Gage } \\
\text { height } \\
\text { (feet) }\end{array}$} & \multicolumn{2}{|c|}{ Discharge } \\
\hline & & & & & & & \multirow[b]{2}{*}{ Cfs } & \multirow{2}{*}{$\begin{array}{c}\text { Recur- } \\
\text { rence } \\
\text { interval } \\
\text { (years) }\end{array}$} \\
\hline & & & Period & Year & & & & \\
\hline \multicolumn{9}{|c|}{ Little Miami River basin-Continued } \\
\hline 21 & $\begin{array}{l}\text { Todd Fork near } \\
\text { Roachester. }\end{array}$ & 219 & $1953-68$ & 1959 & 24 & $\begin{array}{l}19.50 \\
19.49\end{array}$ & $\begin{array}{l}25,500 \\
25,400\end{array}$ & 11.4 \\
\hline \multirow[t]{2}{*}{22} & $\begin{array}{l}\text { Little Miami } \\
\text { River at } \\
\text { Milford. }\end{array}$ & 1,203 & $\begin{array}{c}1913-68 \\
1915-17 \\
1925-36 \\
1939-68\end{array}$ & $\begin{array}{l}1913 \\
1959\end{array}$ & $\cdots$ & $\begin{array}{l}25.5 \\
22.3\end{array}$ & $8 \stackrel{(2)}{(2)}^{-100}$ & $-\cdots$ \\
\hline & & & $1909-00$ & & 24 & 17.75 & 52,200 & 18 \\
\hline \multirow[t]{2}{*}{23} & $\begin{array}{l}\text { East Fork Little } \\
\text { Miami River at }\end{array}$ & 237 & $\begin{array}{c}1949-53 \\
1960-68 .\end{array}$ & 1964 & $\ldots$ & 15.23 & 19,800 & $\ldots$ \\
\hline & Williamsburg. & & & & 24 & 13.43 & 15,400 & 5 \\
\hline \multirow[t]{2}{*}{24} & $\begin{array}{l}\text { East Fork Little } \\
\text { Miami River at }\end{array}$ & 476 & $\begin{array}{l}1915-20 \\
1925-68\end{array}$ & 1964 & - - & 23.84 & 42,400 & - - - \\
\hline & Perintown. & & $\ldots$ & $\ldots$ & 24 & 21.87 & 31,500 & 7 \\
\hline
\end{tabular}

${ }^{1}$ Ratio of peak discharge to 50 -year flood.

$\because$ Undetermined.

${ }^{3}$ Site and datum then in use.

\section{FLOODS OF MAY 29-30 IN SOUTHEASTERN NEW YORK}

By F. LUMAN ROBISON

The most serious flood event of the year in New York occurred in the southeastern part of the State at the end of May.

Very heavy rains of 3.0 to 6.3 inches centered in the extreme southeastern counties caused severe flooding of buildings, streets, highways, crops, and low-lying areas in Ulster, Orange, Westchester, Rockland, and Nassau Counties and in the boroughs of New York City. Thousands of acres of valuable truck-garden crops were inundated in Orange County.

In most areas the heavy rain began about 10 p.m. on May 28 and ended before noon on the next day. The maximum official U.S. Weather Bureau measurement of the storm was 5.54 inches at Dobbs Ferry. At Central Park in New York City a reading of 4.88 inches broke a 60 -year record for 24 -hour rainfall. At Suffern and at Greenwood Lake, unofficial measurements of 6.30 inches and 6.07 inches, respectively, were reported. The extent and distribution of rainfall is shown in figure 10. This storm extended into the area described in the following section on the floods of May 29-31 in northeastern New Jersey.

The intensity of the storm caused streams to rise rapidly. The peak discharge of the Bronx River at Bronxville (No. 5, table 8) was the greatest since establishment of the gaging station in 1944. The peak discharge of the Mamaroneck River at Mamaroneck (No. 3, table 8) was the second highest in the 24- 


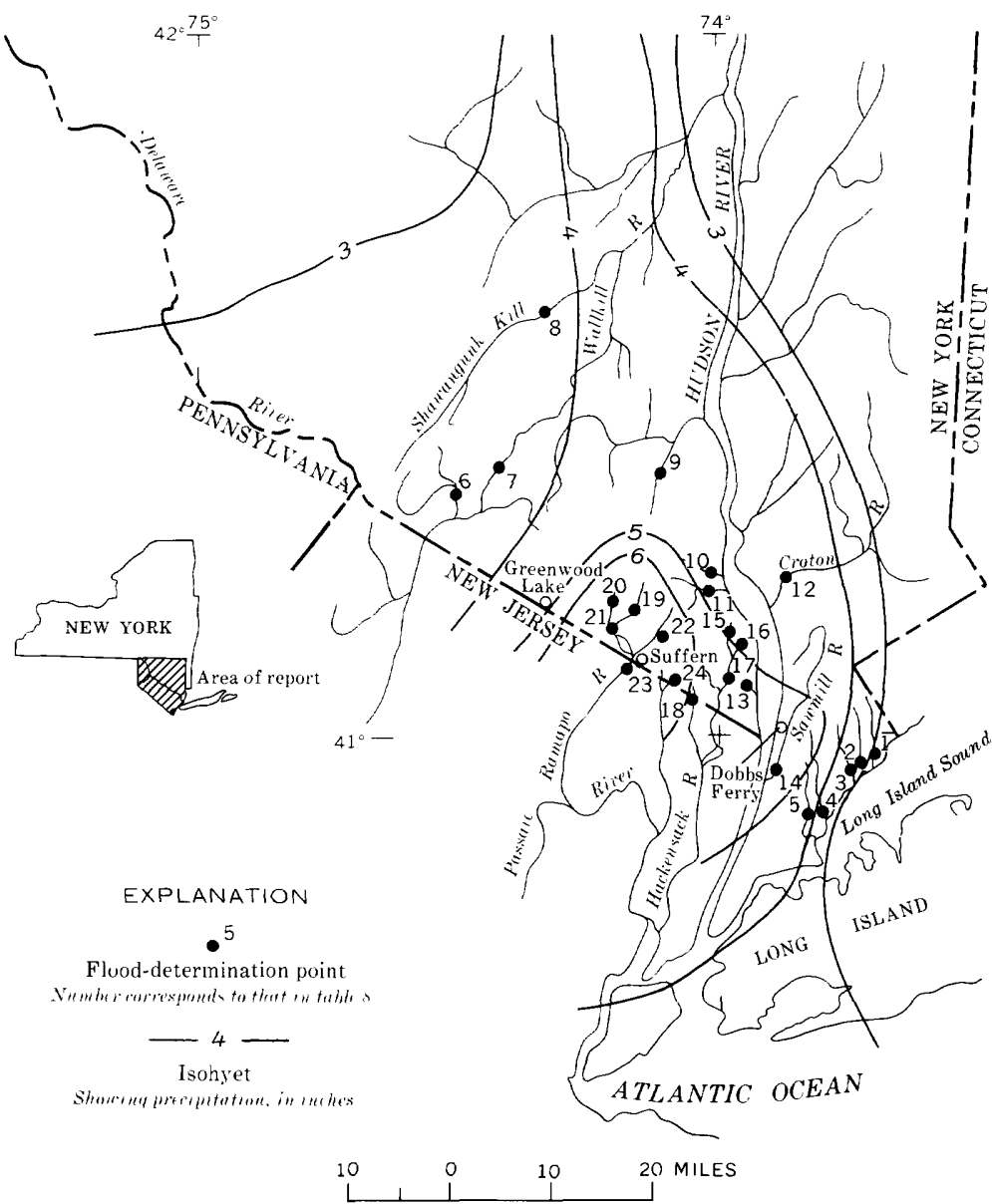

FIgURE 10.-Flood area; location of flood-determination points and isohyets for May 28-29, floods of May 29-30 in southeastern New York.

year record. The only long-term record (50 years) in the Ramapo River basin is near Mahwah, N.J. (No. 23, table 8) about 1 mile below the New York-New Jersey State line, where the peak discharge on May 29 was exceeded only by those of the floods of October 1903 and October 1955. On several small streams in Rockland County, where gages were installed in 1959 and 1960, the discharge from this storm exceeded any previously recorded.

Table 8 contains peak discharges for many streams in the flood area, with corresponding maximum peaks of record prior to May 29 for comparison. Data for some of the May flood peaks were obtained from records at U.S. Geological Survey gaging sta- 
tions while others are the results of indirect measurements of peak discharges made after the floods by Survey engineers. Sites where flood discharges were determined are shown in figure 10.

Flood-frequency relationships are not well defined for the areas affected by these floods. Manmade influences due to urbanization affect the peak discharge for many streams. Gaging-station records for small areas are mostly of short duration; therefore, frequency relations for these small areas are uncertain. On the basis of the regional flood-frequency relationships, the peak discharge of $8,600 \mathrm{cfs}$ on May 29 had a recurrence interval of about 25 years for the long-term station on Ramapo River near Mahwah, N.J., which has a comparatively large drainage area of 118 square miles. A study of the peak discharges listed in table 8 indicates that several were at least equal to that of a 25-year event.

The storm indirectly caused the death of a man from Blauvelt. When the eastbound lane of State Highway 59 was closed by flooding, he was riding his motorcycle east in the westbound lane and collided with a car.

Many streets and cellars were flooded in the villages of Orange and Rockland Counties and several families were forced to leave their homes. The village of Suffern was nearly isolated for a time when both east and west approaches of State Highway 59 were blocked by high water. An indication of the amount of flood damage suffered was the declaration by the U.S. Small Business Administration that Orange and Rockland Counties were disaster areas.

TABLE 8.-Flood stages and discharges, May $29-30$ in southeastern New York

\begin{tabular}{|c|c|c|c|c|c|c|c|}
\hline \multirow{3}{*}{ No. } & \multirow{3}{*}{$\begin{array}{l}\text { Stream and place } \\
\text { of determination }\end{array}$} & \multirow{3}{*}{$\begin{array}{l}\text { Drainage } \\
\text { area } \\
\text { (sq mi) }\end{array}$} & \multicolumn{5}{|c|}{ Maximum floods } \\
\hline & & & \multicolumn{2}{|c|}{$\begin{array}{l}\text { Known before } \\
\text { May } 1968\end{array}$} & \multirow{2}{*}{$\begin{array}{l}\text { May } \\
1968\end{array}$} & \multirow{2}{*}{$\begin{array}{c}\text { Gage } \\
\text { height } \\
\text { (feet) }\end{array}$} & \multirow{2}{*}{$\begin{array}{l}\text { Discharge } \\
\text { (cfs) }\end{array}$} \\
\hline & & & Period & Year & & & \\
\hline \multicolumn{8}{|c|}{ Blind Brook basin } \\
\hline 1 & $\begin{array}{l}\text { Blind Brook at } \\
\text { Rye. }\end{array}$ & 9.20 & $\begin{array}{l}1944-68 \\
----\end{array}$ & $\begin{array}{l}1955 \\
-\end{array}$ & 29 & $\begin{array}{l}9.62 \\
6.68\end{array}$ & $\begin{array}{r}1,360 \\
896 \\
\end{array}$ \\
\hline \multicolumn{8}{|c|}{ Beaver Swamp Brook basin } \\
\hline 2 & $\begin{array}{c}\text { Beaver Swamp } \\
\text { Brook near } \\
\text { Harrison. }\end{array}$ & 4.71 & $1944-68$ & 1962 & $29^{--}$ & $\begin{array}{l}3.09 \\
1.81\end{array}$ & $\begin{array}{r}167 \\
86\end{array}$ \\
\hline \multicolumn{8}{|c|}{ Mamaroneck River basin } \\
\hline \multirow[t]{2}{*}{3} & \multirow[t]{2}{*}{$\begin{array}{l}\text { Mamaroneck River } \\
\text { at Mamaroneck. }\end{array}$} & \multirow[t]{2}{*}{23.4} & \multirow{2}{*}{$\begin{array}{l}1938 \\
1944-52, \\
1955-68 . \\
-\ldots .---\end{array}$} & \multirow{2}{*}{$\begin{array}{l}1938 \\
1944 \\
1955 \\
-\ldots-\end{array}$} & $\begin{array}{l}-\cdots \\
-\cdots\end{array}$ & $\begin{array}{l}111.5 \\
37.82\end{array}$ & $\begin{array}{c}\left({ }^{2}\right) \\
1,940\end{array}$ \\
\hline & & & & & 29 & 5.69 & 1,840 \\
\hline
\end{tabular}

See footnotes at end of tables. 
TABLE 8.-Flond stages and discharges, May 29-30 in southeastern New York-Continued

\begin{tabular}{|c|c|c|c|c|c|c|c|}
\hline \multirow{3}{*}{ No. } & \multirow{3}{*}{$\begin{array}{l}\text { Stream and place } \\
\text { of determination }\end{array}$} & \multirow{3}{*}{$\begin{array}{l}\text { Drainage } \\
\text { area } \\
\text { (sq mi) }\end{array}$} & \multicolumn{5}{|c|}{ Maximum floods } \\
\hline & & & \multicolumn{2}{|c|}{$\begin{array}{l}\text { Known before } \\
\text { May } 1968\end{array}$} & \multirow{2}{*}{$\begin{array}{l}\text { May } \\
1968\end{array}$} & \multirow{2}{*}{$\begin{array}{l}\text { Gage } \\
\text { height } \\
\text { (feet) }\end{array}$} & \multirow{2}{*}{$\begin{array}{c}\text { Dis- } \\
\text { charge } \\
\text { (cfs) }\end{array}$} \\
\hline & & & Period & Year & & & \\
\hline \multicolumn{8}{|c|}{ Hutchinson River basin } \\
\hline 4 & $\begin{array}{l}\text { Hutchinson River } \\
\text { at Pelham. }\end{array}$ & 5.76 & $1944-68$ & 1962 & 29 & $\begin{array}{l}4.98 \\
4.02 \\
\end{array}$ & $\begin{array}{l}390 \\
166 \\
\end{array}$ \\
\hline \multicolumn{8}{|c|}{ Bronx River basin } \\
\hline 5 & $\begin{array}{c}\text { Bronx River at } \\
\text { Bronxville. }\end{array}$ & 2.65 & $\begin{array}{l}1944-68 \\
\ldots \ldots\end{array}$ & $\begin{array}{l}1955 \\
\cdots \\
\end{array}$ & 29 & $\begin{array}{l}6.65 \\
6.35 \\
\end{array}$ & $\begin{array}{r}922 \\
1,450 \\
\end{array}$ \\
\hline \multicolumn{8}{|c|}{ Hudson River basin } \\
\hline 6 & $\begin{array}{l}\text { Rutgers Creek at } \\
\text { Gardnerville. }\end{array}$ & 59.7 & $1944-68$ & 1955 & 30 & $\begin{array}{r}12.38 \\
5.84\end{array}$ & $\begin{array}{l}8,490 \\
1,580\end{array}$ \\
\hline 7 & $\begin{array}{l}\text { Wallkill River at } \\
\text { Pellets Island } \\
\text { Mountain. }\end{array}$ & 385 & $\begin{array}{c}1920-68 \\
\ldots\end{array}$ & 1936 & 30 & $\begin{array}{l}26.0 \\
13.7\end{array}$ & $\begin{array}{r}12,400 \\
3,750\end{array}$ \\
\hline 8 & $\begin{array}{l}\text { Shawangunk Kill } \\
\text { at Pine Bush. }\end{array}$ & 102 & $\begin{array}{l}1925-32 \\
1952, \\
1955-68 .\end{array}$ & 1955 & 29 & $\begin{array}{l}12.5 \\
10.03\end{array}$ & $\begin{array}{l}9,700 \\
6,840\end{array}$ \\
\hline 9 & $\begin{array}{l}\text { Woodbury Creek } \\
\text { near Highland } \\
\text { Mills. }\end{array}$ & 11.2 & $\begin{array}{l}1955-68 \\
-\ldots .\end{array}$ & $\begin{array}{l}1967 \\
1967\end{array}$ & 29 & $\begin{array}{l}3.04 \\
4.97\end{array}$ & 150 \\
\hline 10 & $\begin{array}{l}\text { Cedar Pond Creek } \\
\text { at Stony Point. }\end{array}$ & 17.3 & $1959-68$ & 1961 & 29 & $\begin{array}{l}4.95 \\
8.87\end{array}$ & $\begin{array}{r}830 \\
1,920\end{array}$ \\
\hline 11 & $\begin{array}{l}\text { South Branch } \\
\text { Minisceongo } \\
\text { Creek at } \\
\text { Letchworth } \\
\text { Village }\end{array}$ & 5.83 & $1960-68$ & $\begin{array}{c}1960 \\
-\ldots\end{array}$ & 29 & $\begin{array}{l}3.13 \\
3.84\end{array}$ & $\begin{array}{l}120 \\
230\end{array}$ \\
\hline 12 & $\begin{array}{l}\text { Croton River at } \\
\text { New Croton Dam } \\
\text { near Croton-on- } \\
\text { Hudson. }\end{array}$ & 378 & $1934-68$ & 1955 & 30 & $\begin{array}{r}18.44 \\
6.69\end{array}$ & $\begin{array}{r}45,400 \\
4,740\end{array}$ \\
\hline 13 & $\begin{array}{l}\text { Sparkill Creek at } \\
\text { Sparkill. }\end{array}$ & 11.1 & $1960-68$ & 1962 & $29^{-}$ & $\begin{array}{l}3.86 \\
5.34\end{array}$ & $\begin{array}{l}438 \\
830\end{array}$ \\
\hline 14 & $\begin{array}{l}\text { Sawmill River at } \\
\text { Yonkers. }\end{array}$ & 25.6 & $\begin{array}{r}1944-68 \\
-\end{array}$ & 1955 & 30 & $\begin{array}{l}5.34 \\
4.65 \\
\end{array}$ & $\begin{array}{l}890 \\
481\end{array}$ \\
\hline \multicolumn{8}{|c|}{ Hackensack River basin } \\
\hline 15 & $\begin{array}{l}\text { Hackensack River } \\
\text { at Brookside } \\
\text { Park. }\end{array}$ & 13.2 & $\begin{array}{l}1959-63 \\
1966-68 .\end{array}$ & 1960 & 29 & $\begin{array}{l}6.67 \\
7.40\end{array}$ & $\begin{array}{l}1,010 \\
1,350\end{array}$ \\
\hline 16 & $\begin{array}{l}\text { East Branch } \\
\text { Hackensack } \\
\text { river at Congers. }\end{array}$ & 6.86 & $1959-68$ & 1960 & 29 & $\begin{array}{l}9.12 \\
9.29\end{array}$ & $\begin{array}{l}110 \\
125\end{array}$ \\
\hline 17 & $\begin{array}{l}\text { Hackensack River } \\
\text { at West Nyack. }\end{array}$ & 29.4 & $\begin{array}{l}1959-68 \\
-\ldots\end{array}$ & 1960 & 29 & $\begin{array}{l}6.23 \\
6.84\end{array}$ & $\begin{array}{l}654 \\
797\end{array}$ \\
\hline 18 & $\begin{array}{c}\text { Pascack Brook } \\
\text { tributary at } \\
\text { Spring Valley. }\end{array}$ & 4.58 & $1960-68$ & 1960 & $29^{--}$ & $\begin{array}{l}4.46 \\
5.16\end{array}$ & $\begin{array}{l}378 \\
580\end{array}$ \\
\hline \multicolumn{8}{|c|}{ Passaic River basin } \\
\hline 19 & $\begin{array}{l}\text { Ramapo River at } \\
\text { Sloatsburg. }\end{array}$ & 60.9 & $\begin{array}{l}1955 \\
1959-68 \\
\cdots\end{array}$ & $\begin{array}{l}1955 \\
1960 \\
-\end{array}$ & 29 & $\begin{array}{l}12.2 \\
9.52 \\
11.4\end{array}$ & $\begin{array}{l}6,240 \\
2,270 \\
4,600\end{array}$ \\
\hline 20 & $\begin{array}{l}\text { Stony Brook at } \\
\text { Sloatsburg. }\end{array}$ & 18.2 & $1960-68$ & 1960 & $29^{--}$ & $\begin{array}{l}3.14 \\
5.23\end{array}$ & $\begin{array}{l}1,060 \\
1,650\end{array}$ \\
\hline 21 & $\begin{array}{l}\text { Ramapo River } \\
\text { tributary at } \\
\text { Sloatsburg. }\end{array}$ & 5.35 & $\begin{array}{l}1959-68 \\
-\end{array}$ & 1960 & 29 & $\begin{array}{r}7.05 \\
10.13\end{array}$ & $\begin{array}{l}180 \\
540\end{array}$ \\
\hline 22 & $\begin{array}{c}\text { Mahwah River } \\
\text { near Suffern. }\end{array}$ & 12.3 & $1958-68$ & 1960 & 29 & $\begin{array}{l}5.76 \\
7.79\end{array}$ & $\begin{array}{r}462 \\
1,650\end{array}$ \\
\hline
\end{tabular}

See footnotes at end of table. 
TABLE 8.-Flood stages and discharges, May 29-30 in southeastern New York-Continued

\begin{tabular}{|c|c|c|c|c|c|c|c|}
\hline \multirow{3}{*}{ No. } & \multirow{3}{*}{$\begin{array}{l}\text { Stream and place } \\
\text { of determination }\end{array}$} & \multirow{3}{*}{$\begin{array}{c}\text { Drainage } \\
\text { area } \\
\text { (sq mi) }\end{array}$} & \multicolumn{5}{|c|}{ Maximum floods } \\
\hline & & & \multicolumn{2}{|c|}{$\begin{array}{l}\text { Known before } \\
\text { May } 1968\end{array}$} & \multirow{2}{*}{$\begin{array}{l}\text { May } \\
1968\end{array}$} & \multirow{2}{*}{$\begin{array}{c}\text { Gage } \\
\text { height } \\
\text { (feet) }\end{array}$} & \multirow{2}{*}{$\begin{array}{c}\text { Dis- } \\
\text { charge } \\
\text { (cfs) }\end{array}$} \\
\hline & & & Period & Year & & & \\
\hline \multicolumn{8}{|c|}{ Passaic River basin-Continued } \\
\hline \multirow[t]{2}{*}{23} & \multirow{2}{*}{$\begin{array}{l}\text { Ramapo River } \\
\text { near Mahwah, } \\
\text { N. J. }\end{array}$} & \multirow[t]{2}{*}{118} & \multirow{2}{*}{$\begin{array}{l}1903-06, \\
1923-68 . \\
\end{array}$} & 1903 & $\ldots$ & 11.0 & 12,400 \\
\hline & & & & $\ldots$ & 29 & 11.87 & 8,600 \\
\hline 24 & $\begin{array}{l}\text { Pine Brook near } \\
\text { Spring Valley. }\end{array}$ & 2.28 & $1960-68$ & 1962 & 29 & $\begin{array}{l}2.89 \\
3.42\end{array}$ & $\begin{array}{l}120 \\
170\end{array}$ \\
\hline
\end{tabular}

1 Affected by hurricane wave.

2 Unknown.

3 At datum 0.41 foot higher than present datum.

4 Affected by backwater from ice.

5 At site 250 feet downstream at different datum.

\section{FLOODS OF MAY 29-31 IN NORTHEASTERN NEW JERSEY}

Extremely heavy rains over the Passaic River basin and adjoining areas caused major flooding in northeastern New Jersey (fig. 11). The rainfall occurred on May 28-30, with much of the precipitation measured on May 29. The heaviest rains evidently occurred on the ridge around Chatham, where a total of 7.07 inches was reported. The second highest rainfall of 6.67 inches was reported at Charlottesburg. This storm extended into the area described in the preceding section on the floods of May 29-30 in southeastern New York. Peak discharges at gaging stations in the Hackensack, Saddle, and Rahway River basins and on the Passaic River near Chatham were equal to or greater than those of a 50-year flood. The peak discharge of 2,560 $\mathrm{cfs}$ at Chatham (No. 5, table 9) was the highest since January 1905. Maximum discharges for the period of record (since 1922 at some stations) occurred at the gaging stations in the Hackensack and Rahway River basins. (See table 9.)

Northeastern New Jersey was declared a disaster area. More than 2,000 families were evacuated from flooded areas. The Small Business Administration estimated the flood damage at $\$ 133$ million. Seven deaths occurred due to drowning. 


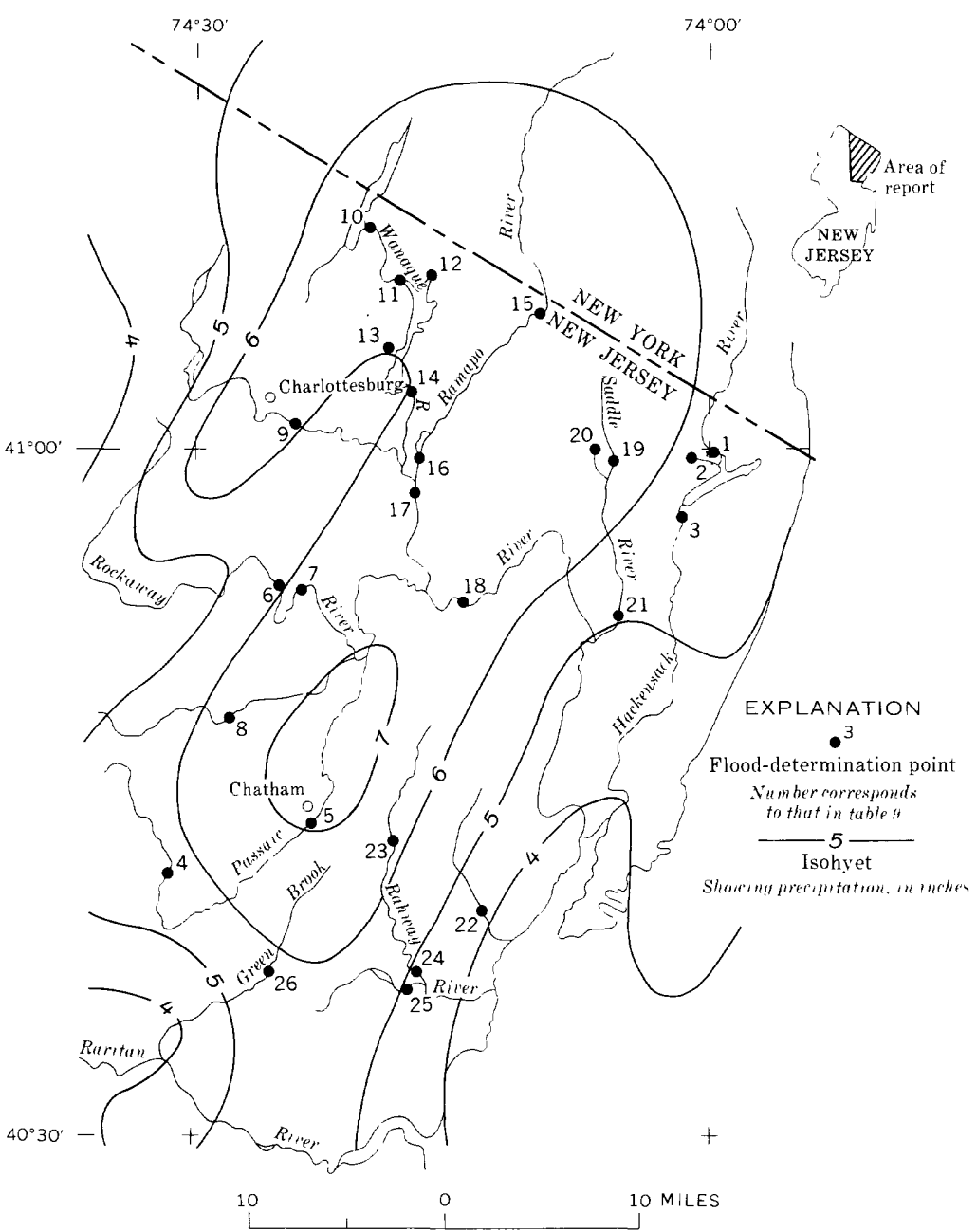

Figure 11.-Flood area; location of flood determination points and isohyets for May 28-30, floods of May 29-31 in northeastern New Jersey. 
TABLE 9.-Flood stages and discharges, May 29-31 in northeastern New Jersey

\begin{tabular}{|c|c|c|c|c|c|c|c|c|}
\hline \multirow{4}{*}{ No. } & \multirow{4}{*}{$\begin{array}{l}\text { Stream and place } \\
\text { of determination }\end{array}$} & \multirow{4}{*}{$\begin{array}{l}\text { Drainage } \\
\text { area } \\
(\mathrm{sq} \mathrm{mi})\end{array}$} & \multicolumn{6}{|c|}{ Maximum floods } \\
\hline & & & \multirow{2}{*}{\multicolumn{2}{|c|}{$\begin{array}{l}\text { Known before } \\
\text { May } 1968\end{array}$}} & \multirow{3}{*}{$\begin{array}{l}\text { May } \\
1968\end{array}$} & \multirow{3}{*}{$\begin{array}{c}\text { Gage } \\
\text { height } \\
\text { (feet) }\end{array}$} & \multirow[b]{3}{*}{ Cfs } & harge \\
\hline & & & & & & & & \multirow{2}{*}{$\begin{array}{c}\text { Recur- } \\
\text { rence } \\
\text { interval } \\
\text { (years) }\end{array}$} \\
\hline & & & Period & Year & & & & \\
\hline \multicolumn{9}{|c|}{ Hackensack River basin } \\
\hline 1 & $\begin{array}{c}\text { Hackensack } \\
\text { River at } \\
\text { Rivervale. }\end{array}$ & 58.0 & $1942-68$ & 1955 & 29 & $\begin{array}{l}6.03 \\
6.23\end{array}$ & $\begin{array}{l}1,450 \\
1,500\end{array}$ & $\overline{50}$ \\
\hline 2 & $\begin{array}{l}\text { Pascack Brook } \\
\text { at Westwood. }\end{array}$ & 29.6 & $1935-68$ & 1945 & 29 & $\begin{array}{l}6.76 \\
6.61\end{array}$ & $\begin{array}{l}1,610 \\
1,640\end{array}$ & 30 \\
\hline 3 & $\begin{array}{l}\text { Hackensack } \\
\text { River at } \\
\text { New Milford. }\end{array}$ & 113 & $\begin{array}{c}1922-68 \\
-\end{array}$ & 1951 & 30 & $\begin{array}{l}6.14 \\
6.60\end{array}$ & $\begin{array}{l}3,660 \\
4,040\end{array}$ & $50^{-}$ \\
\hline \multicolumn{9}{|c|}{ Passaic River basin } \\
\hline 4 & $\begin{array}{l}\text { Passaic River } \\
\text { near Milling- } \\
\text { ton. }\end{array}$ & 55.4 & $\begin{array}{c}1904-06 \\
1922-68\end{array}$ & 1905 & 29 & $\begin{array}{l}17.8 \\
8.04\end{array}$ & $\begin{array}{l}2,000 \\
1,0,70\end{array}$ & -- \\
\hline 5 & $\begin{array}{l}\text { Passaic River } \\
\text { near Chatham. }\end{array}$ & 100 & $\begin{array}{c}1903-11 \\
1938-68\end{array}$ & 1905 & 29 & $\begin{array}{l}28.3 \\
7.90\end{array}$ & $\begin{array}{l}3,000 \\
2,560\end{array}$ & 31.07 \\
\hline 6 & $\begin{array}{l}\text { Rockaway River } \\
\text { above reservoir } \\
\text { at Boonton. }\end{array}$ & 116 & $\begin{array}{l}1938-68 \\
-\end{array}$ & $\begin{array}{l}1952 \\
-\cdots\end{array}$ & 29 & $\begin{array}{l}6.62 \\
6.22\end{array}$ & $\begin{array}{l}3,250 \\
3,020\end{array}$ & 10 \\
\hline 7 & $\begin{array}{l}\text { Rockaway River } \\
\text { below reservoir } \\
\text { at Boonton. }\end{array}$ & 119 & $\begin{array}{c}1903 \\
1906-68\end{array}$ & 1903 & 29 & 8.20 & $\begin{array}{r}4,560 \\
3,450\end{array}$ & $\left({ }^{5}\right)$ \\
\hline 8 & $\begin{array}{l}\text { Whippany River } \\
\text { at Morristown. }\end{array}$ & 29.4 & $1922-68$ & 1928 & $29^{--}$ & $\begin{array}{l}7.3 \\
5.16\end{array}$ & $\begin{array}{r}2,000 \\
956\end{array}$ & 3 \\
\hline 9 & $\begin{array}{l}\text { Pequannock River } \\
\text { at Macopin In- } \\
\text { take Dam. }\end{array}$ & 63.7 & $1898-1968$ & 1903 & 29 & $\begin{array}{l}3.85 \\
2.15\end{array}$ & $\begin{array}{l}6,100 \\
2,510\end{array}$ & (5) \\
\hline 10 & $\begin{array}{l}\text { Wanaque River } \\
\text { at Awosting. }\end{array}$ & 27.1 & $1919-68$ & 1955 & 29 & $\begin{array}{l}5.85 \\
4.99\end{array}$ & $\begin{array}{r}1,300 \\
875\end{array}$ & 8 \\
\hline 11 & $\begin{array}{l}\text { Wanaque River } \\
\text { at Monks. }\end{array}$ & 40.4 & $1935-68$ & 1955 & $29^{--}$ & $\begin{array}{l}4.15 \\
3.97\end{array}$ & $\begin{array}{l}3,640 \\
3,330\end{array}$ & 30 \\
\hline 12 & $\begin{array}{c}\text { Ringwood Creek } \\
\text { near Wanaque. }\end{array}$ & 19.1 & $1935-68$ & 1951 & 29 & $\begin{array}{l}3.74 \\
3.72\end{array}$ & $\begin{array}{l}1,150 \\
1,020\end{array}$ & $\overline{\mathbf{5}}^{--}$ \\
\hline 13 & $\begin{array}{l}\text { West Brook near } \\
\text { Wanaque. }\end{array}$ & 11.8 & $1935-68$ & 1951 & 29 & $\begin{array}{l}6.6 \\
4.35\end{array}$ & $\begin{array}{r}1,900 \\
818\end{array}$ & $\overline{5}$ \\
\hline 14 & $\begin{array}{l}\text { Wanaque River } \\
\text { at Wanaque. }\end{array}$ & 9.04 & $\begin{array}{c}1913-15 \\
1919-68 \\
.\end{array}$ & 1951 & 29 & $\begin{array}{l}9.12 \\
8.42\end{array}$ & $\begin{array}{l}8,470 \\
5,920\end{array}$ & $(5)$ \\
\hline 15 & $\begin{array}{l}\text { Ramapo River } \\
\text { near Mahwah. }\end{array}$ & 118 & $\begin{array}{c}1903-06, \\
1923-68 .\end{array}$ & 1903 & 29 & $\begin{array}{l}{ }^{6} 11.0 \\
11.87\end{array}$ & $\begin{array}{r}12,400 \\
7,740\end{array}$ & 12 \\
\hline 16 & $\begin{array}{l}\text { Ramapo River at } \\
\text { Pompton } \\
\text { Lakes. }\end{array}$ & 160 & $1922-68$ & $\begin{array}{l}1936 \\
1955\end{array}$ & 29 & $\begin{array}{l}4.40 \\
3.82\end{array}$ & $\begin{array}{r}12,300 \\
9,530\end{array}$ & $12^{-}$ \\
\hline 17 & $\begin{array}{l}\text { Pompton River at } \\
\text { Pompton } \\
\text { Plains. }\end{array}$ & 355 & $\begin{array}{c}1903-04 \\
1940-68\end{array}$ & 1903 & 30 & $\begin{array}{l}{ }^{\top} 14.3 \\
22.18\end{array}$ & $\begin{array}{l}28,340 \\
13,100\end{array}$ & 15 \\
\hline 18 & $\begin{array}{l}\text { Passaic River at } \\
\text { Little Falls. }\end{array}$ & 762 & $1898-1968$ & 1903 & 31 & 10.73 & $\begin{array}{r}28,000 \\
13,500\end{array}$ & 10 \\
\hline 19 & $\begin{array}{l}\text { Saddle River at } \\
\text { Ridgewood. }\end{array}$ & 21.6 & $\begin{array}{l}1945 \\
1955-68 \\
\ldots \ldots\end{array}$ & $\begin{array}{l}1945 \\
1955\end{array}$ & 29 & $\begin{array}{l}8.88 \\
9.80\end{array}$ & $\begin{array}{l}6,400 \\
1,510 \\
2,060\end{array}$ & 31.05 \\
\hline 20 & $\begin{array}{l}\text { Hohokus Brook at } \\
\text { Hohokus. }\end{array}$ & 16.4 & $1954-68$ & 1955 & 29 & $\begin{array}{l}4.50 \\
4.31\end{array}$ & $\begin{array}{l}2,350 \\
2,120\end{array}$ & 24 \\
\hline 21 & $\begin{array}{l}\text { Saddle River at } \\
\text { Lodi. }\end{array}$ & 54.6 & $\begin{array}{l}1924-68 \\
\ldots\end{array}$ & 1945 & 29 & $\begin{array}{r}10.00 \\
9.53\end{array}$ & $\begin{array}{l}3,500 \\
3,330\end{array}$ & $3 \overline{1.06}$ \\
\hline \multicolumn{9}{|c|}{ Elizabeth River basin } \\
\hline 22 & $\begin{array}{l}\text { Elizabeth River at } \\
\text { Elizabeth. }\end{array}$ & 20.2 & $1922-68$ & $\begin{array}{l}1938 \\
1966\end{array}$ & $29^{--}$ & $\begin{array}{r}816.19 \\
15.09\end{array}$ & $\begin{array}{l}2,720 \\
2,450\end{array}$ & 13 \\
\hline
\end{tabular}

See footnotes at end of table. 
TABLE 9.-Flood stages aud discharges, May 29-31 in northeastern New Jersey-Continued

\begin{tabular}{|c|c|c|c|c|c|c|c|c|}
\hline \multirow{4}{*}{ No. } & \multirow{4}{*}{$\begin{array}{l}\text { Stream and place } \\
\text { of determination }\end{array}$} & \multirow{4}{*}{$\begin{array}{l}\text { Drainage } \\
\text { area } \\
(\mathrm{sq} \mathrm{mi})\end{array}$} & \multicolumn{6}{|c|}{ Maximum floods } \\
\hline & & & \multirow{2}{*}{\multicolumn{2}{|c|}{$\begin{array}{c}\text { Known before } \\
\text { May } 1968\end{array}$}} & \multirow{3}{*}{$\begin{array}{l}\text { May } \\
1968\end{array}$} & \multirow{3}{*}{$\begin{array}{c}\text { Gage } \\
\text { height } \\
\text { (feet) }\end{array}$} & \multirow[b]{3}{*}{ Cfs } & \multirow{3}{*}{$\begin{array}{c}\text { charge } \\
\text { Recur- } \\
\text { rence } \\
\text { interval } \\
\text { (years) }\end{array}$} \\
\hline & & & & & & & & \\
\hline & & & Period & Year & & & & \\
\hline \multicolumn{9}{|c|}{ Rahway River basin } \\
\hline 23 & $\begin{array}{l}\text { Rahway River } \\
\text { near } \\
\text { Springfield. }\end{array}$ & 25.5 & $1938-68$ & 1938 & 29 & $\begin{array}{l}7.51 \\
8.50\end{array}$ & $\begin{array}{l}1,940 \\
2,520\end{array}$ & $3 \overline{1.37}$ \\
\hline 24 & $\begin{array}{l}\text { Rahway River at } \\
\text { Rahway. }\end{array}$ & 40.9 & $1922-68$ & 1938 & $29^{--}$ & $\begin{array}{l}6.35 \\
6.67\end{array}$ & $\begin{array}{l}3,140 \\
3,530\end{array}$ & $3 \overline{1.04}$ \\
\hline 25 & $\begin{array}{c}\text { Robinsons Branch } \\
\text { Rahway River } \\
\text { at Rahway. }\end{array}$ & 21.6 & $1940-68$ & 1953 & $\overline{29}$ & $\begin{array}{l}5.36 \\
6.00\end{array}$ & $\begin{array}{l}1,490 \\
2,550\end{array}$ & 31.25 \\
\hline \multicolumn{9}{|c|}{ Raritan River basin } \\
\hline 26 & $\begin{array}{l}\text { Green Brook at } \\
\text { Plainfield. }\end{array}$ & 9.75 & $1938-68$ & 1938 & $\overline{29}$ & $\begin{array}{r}5.82 \\
-\end{array}$ & $\begin{array}{l}2,890 \\
2,800\end{array}$ & $\left({ }^{5}\right)$ \\
\hline
\end{tabular}

1 At site 0.75 mile downstream at different datum.

2 At bridge 150 feet upstream at different datum.

3 Ratio of peak discharge to 50-year flood.

4 Daily discharge.

5 Not determined.

At site 250 feet downstream at different datum.

it site, 1,600 feet upstream at different datum.

8 Affected by debris.

a Part of flow at extreme high stages bypasses the station; amount undetermined.

\section{FLOODS OF JUNE AND AUGUST IN WYOMING}

By D. J. O'CONNELL

Numerous floods occurred throughout Wyoming in June and August. A combination of snowmelt and rainfall produced major floods in June on streams which flow easterly from the Bighorn Mountains in the Yellowstone River basin in north-central Wyoming and on streams which flow northeasterly from the Uinta Mountains in the Green River basin in southwestern Wyoming. Also, locally heavy thunderstorms produced significant floods in the north-central and southwestern parts of the State in June and August. Stages and discharges for these floods are listed by basin in downstream order in table 10 .

\section{NORTH-CENTRAI, WYOMING;}

Floods in June in north-central Wyoming were the result of a combination of rainfall and snowmelt runoff on streams flowing easterly from the Bighorn Mountains and were the result of general rains and locally intense thunderstorms on streams flowing westerly from Big Horn Mountains (fig. 12).

The snowpack on the Bighorn Mountains was decreasing rapidly when the rains began on June 4 . The easterly flowing streams were not yet in their recession phase, but the westerly 


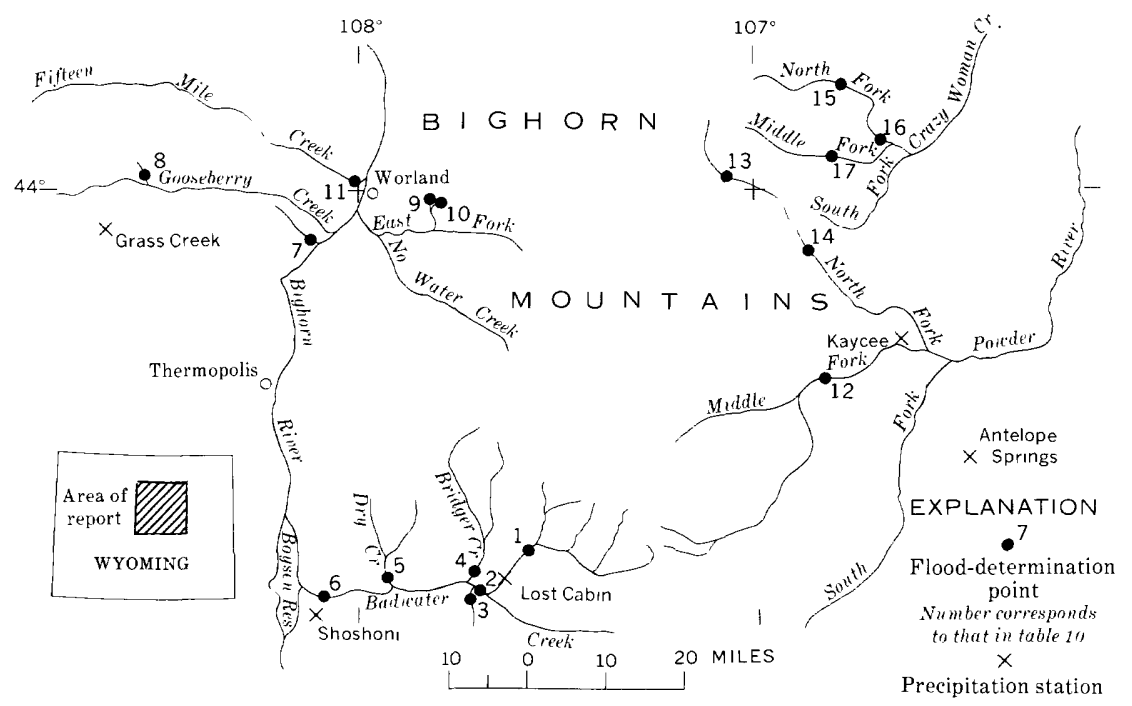

FIGURE 12.-Flood area; location of flood-determination points and precipitation stations, floods of June and August in north-central Wyoming.

flowing streams were well down in their recession phase. The amounts of rainfall recorded at nearby U.S. Weather Bureau stations, in inches, are tabulated below:

\begin{tabular}{lccccc}
\hline June & Antelope Springs & Kaycee & Lost Cabin & Shoshoni \\
\hline 4 & 0 & .45 & .58 & 0.06 & 0.02 \\
5 & 1.01 & 1.14 & .23 & .25 \\
6 & .17 & .03 & 1.15 & .50 \\
7 & .10 & .20 & Trace & .07 \\
8 & .25 & .64 & .98 & 0 \\
9 & 1.98 & 2.63 & .87 & .23 \\
\hline
\end{tabular}

The rains caused rapid melting of the remaining snowpack and the consequent runoff caused several peak discharges of record at gaging stations on streams flowing in an easterly direction (Nos. 12-17, table 10). The rain received by June 6 increased the soil moisture on drainages for streams flowing in a westerly direction and produced minor peaks in Badwater Creek. Additional rain on June 8 and 9 and favorable antecedent runoff conditions caused peak discharges of record at six gaging sites on Badwater Creek and its tributaries (Nos. 1-6, table 10). 
Scouring of the banks along Badwater Creek during the flood endangered the tracks of the Chicago, Burlington \& Quincy Railroad near the town of Bonneville and caused minor concern but no delays in rail traffic. Other damage from floodwater was limited to rural areas and was minor.

A general rainstorm in the Thermopolis-Worland area on August 23 seemingly reached its greatest intensity in the headwaters of Little Gooseberry Creek. The sites where peak discharges were determined are indicated in figure 12 (Nos. 7-11), and discharges are shown in table 10. The U.S. Weather Bureau stations at Grass Creek and at Worland Airport measured 1.32 and 1.12 inches of precipitation, respectively, on August 23. The peak discharge on Little Gooseberry Creek (No. 7, table 10) was 2.2 times that of the 50-year flood. The Chicago, Burlington \& Quincy Railroad bridge over Little Gooseberry Creek was damaged and rail traffic was disrupted.

\section{SOUTHWESTERN WYOMING}

Floods in June in southwestern Wyoming resulted from a combination of rainfall and snowmelt runoff on streams which flow northeasterly from the Uinta Mountains (fig. 13). The Uinta Mountains were overlain with a heavy snowpack when the rains began on June 4. The amounts of rainfall recorded at nearby U.S. Weather Bureau stations, in inches, are tabulated below:

\begin{tabular}{|c|c|c|c|c|}
\hline Date & Mountain View & Church Buttes & Green River & Manila \\
\hline \multicolumn{5}{|l|}{ June } \\
\hline $4 \ldots$ & 0 & 0 & Trace & 0.10 \\
\hline $5 \ldots \ldots \ldots$ & 1.11 & .81 & 0.62 & .88 \\
\hline $6 \ldots$ & .18 & .39 & .22 & .22 \\
\hline $7 \ldots$ & 0 & Trace & .02 & .14 \\
\hline 8 & .09 & 0 & .14 & .07 \\
\hline $9 \ldots$ & 0 & .20 & .03 & .05 \\
\hline $10 \ldots \ldots$ & .27 & .15 & Trace & .04 \\
\hline Total & 1.65 & 1.55 & 1.03 & 1.50 \\
\hline \multicolumn{5}{|l|}{ Aug. } \\
\hline $9 \ldots$ & .43 & .41 & .02 & .67 \\
\hline $10 \ldots \ldots$ & .24 & .36 & .54 & 0 \\
\hline $11 \ldots \ldots$ & .03 & Trace & .15 & .15 \\
\hline Total _..... & .70 & .77 & .71 & .82 \\
\hline
\end{tabular}




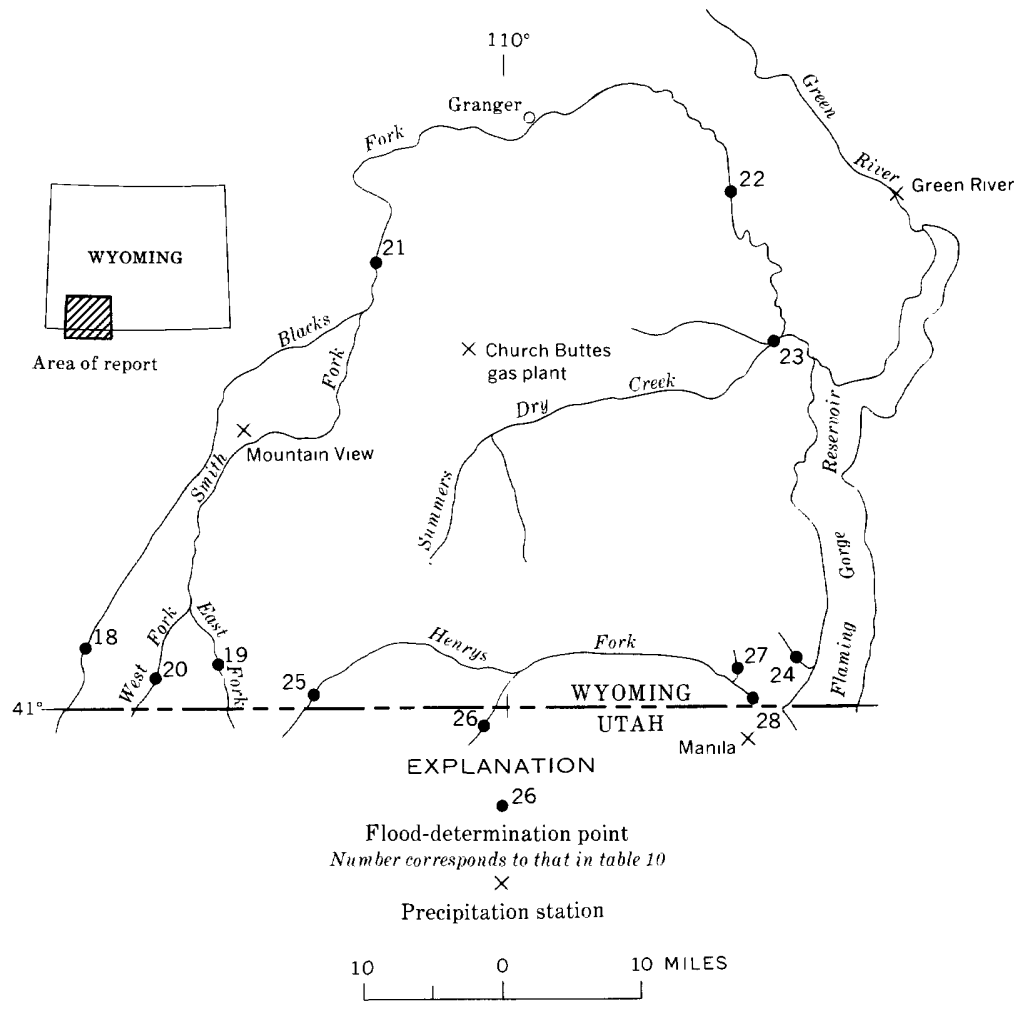

FigURE 13.-Flood area; location of flood-determination points and precipitation stations, floods of June and August in southwestern Wyoming.

Rain and snowmelt runoff combined to produce peaks that were greater than that of the 50-year flood at two of the streamgaging stations (Nos. 20, 25; table 10).

Floodwaters caused minor damage to property in Mountain View and in rural areas on the flood plains. A bridge was damaged at Mountain View.

A rainstorm over southwestern Wyoming produced significant flood peaks at three partial record stations (Nos. 23, 24, 27, table 10) and at one recording station (No. 28, table 10) on August 11. Maximum peaks of record were recorded at two of the partial record stations (Nos. 23, 27 ; table 10). No damage was reported from this flood. 
TABLE 10.-Flood stages and discharges, June and August, in Wyoming

\begin{tabular}{|c|c|c|c|c|c|c|c|c|}
\hline \multirow{4}{*}{ No. } & \multirow{4}{*}{$\begin{array}{l}\text { Stream and place } \\
\text { of determination }\end{array}$} & \multirow{4}{*}{$\begin{array}{l}\text { Drainage } \\
\text { area } \\
\text { (sq mi) }\end{array}$} & \multicolumn{6}{|c|}{ Maximum floods } \\
\hline & & & \multirow{2}{*}{\multicolumn{2}{|c|}{$\begin{array}{c}\text { Known before } \\
\text { June } 1968\end{array}$}} & \multirow{3}{*}{$\begin{array}{c}\text { June, } \\
\text { August } \\
1968\end{array}$} & \multirow{3}{*}{$\begin{array}{c}\text { Gage } \\
\text { height } \\
\text { (feet) }\end{array}$} & \multicolumn{2}{|c|}{ Discharge } \\
\hline & & & & & & & \multirow[b]{2}{*}{ Cfs } & \multirow{2}{*}{$\begin{array}{l}\text { Recur- } \\
\text { rence } \\
\text { interval } \\
\text { (years) }\end{array}$} \\
\hline & & & Period & Year & & & & \\
\hline \multicolumn{9}{|c|}{ Yellowstone River basin } \\
\hline 1 & $\begin{array}{l}\text { Badwater Creek } \\
\text { at Lybyer } \\
\text { Ranch near } \\
\text { Lost Cabin. }\end{array}$ & 131 & $\begin{array}{l}1949-68 \\
-\end{array}$ & $\begin{array}{l}1952 \\
1967 \\
-\end{array}$ & June 9 & $\begin{array}{r}18.88 \\
5.16 \\
6.18\end{array}$ & $\begin{array}{r}708 \\
1,060\end{array}$ & 4 \\
\hline 2 & $\begin{array}{l}\text { Badwater Creek } \\
\text { at Lysite. }\end{array}$ & 415 & $\begin{array}{l}1966-68 \\
-\ldots-\end{array}$ & $\begin{array}{l}1967 \\
---\end{array}$ & June 8 & $\begin{array}{l}6.55 \\
7.92\end{array}$ & $\begin{array}{l}2,260 \\
6,240\end{array}$ & 20 \\
\hline 3 & $\begin{array}{l}\text { Badwater Creek } \\
\text { tributary near } \\
\text { Lysite. }\end{array}$ & 5.86 & $1965-68$ & 1967 & June 8 & $\begin{array}{l}4.10 \\
5.69\end{array}$ & $\begin{array}{r}512 \\
1,460\end{array}$ & $2 \overline{1.8}$ \\
\hline 4 & $\begin{array}{l}\text { Bridger Creek } \\
\text { near Lysite. }\end{array}$ & 182 & $\begin{array}{l}1966-68 \\
-\end{array}$ & 1967 & June 9 & $\begin{array}{l}6.80 \\
7.52\end{array}$ & $\begin{array}{l}727 \\
880\end{array}$ & 3 \\
\hline 5 & $\begin{array}{l}\text { Dry Creek near } \\
\text { Bonneville. }\end{array}$ & 52.6 & $\begin{array}{l}1966-68 \\
-\ldots-\end{array}$ & 1967 & June 9 & $\begin{array}{r}12.41 \\
39.64\end{array}$ & $\begin{array}{r}1,650 \\
584\end{array}$ & 4 \\
\hline 6 & $\begin{array}{l}\text { Badwater Creek } \\
\text { at Bonneville. }\end{array}$ & 808 & $\begin{array}{l}1923 \\
1947-68\end{array}$ & $\begin{array}{l}1923 \\
1956 \\
-\end{array}$ & June 9 & $\begin{array}{l}\left({ }^{4}\right) \\
6.60 \\
7.64\end{array}$ & $\begin{array}{r}18,600 \\
7,260 \\
8,490\end{array}$ & $\begin{array}{l}\cdots \\
16\end{array}$ \\
\hline 7 & $\begin{array}{l}\text { Little Gooseberry } \\
\text { Creek near } \\
\text { Worland. }\end{array}$ & 30.2 & $\ldots \ldots$ & $\ldots$ & Aug. 23 & $\cdots$ & 1,980 & ${ }^{2} 2.2$ \\
\hline 8 & $\begin{array}{l}\text { Murphy Draw } \\
\text { near Grass } \\
\text { Creek. }\end{array}$ & 2.32 & $1965-68$ & 1967 & Aug. 23 & $\begin{array}{l}6.05 \\
4.90\end{array}$ & $\begin{array}{l}583 \\
244\end{array}$ & $\left({ }^{4}\right)$ \\
\hline 9 & $\begin{array}{l}\text { North Prong East } \\
\text { Fork No Water } \\
\text { Creek near } \\
\text { Worland. }\end{array}$ & 3.8 & $\begin{array}{l}1964-68 \\
-\ldots\end{array}$ & $\begin{array}{l}1967 \\
---\end{array}$ & Aug. 23 & $\begin{array}{l}5.46 \\
4.96\end{array}$ & $\begin{array}{l}391 \\
242\end{array}$ & $(4)$ \\
\hline 10 & $\begin{array}{l}\text { North Prong } \\
\text { East Fork No } \\
\text { Water Creek } \\
\text { tributary near } \\
\text { Worland. }\end{array}$ & 2.1 & $1965-68$ & 1967 & Aug. 23 & $\begin{array}{l}4.43 \\
4.00\end{array}$ & ${ }^{(4)} 114$ & $\left({ }^{4}\right)$ \\
\hline 11 & $\begin{array}{l}\text { Fifteen Mile } \\
\text { Creek near } \\
\text { Worland. }\end{array}$ & 518 & $1951-68$ & $\begin{array}{l}1952 \\
-\ldots\end{array}$ & Aug. 23 & $\begin{array}{r}55.77 \\
5.11\end{array}$ & $\begin{array}{l}3,300 \\
1,540\end{array}$ & 3 \\
\hline 12 & $\begin{array}{l}\text { Middle Fork } \\
\text { Powder River } \\
\text { above Kaycee. }\end{array}$ & 450 & $1949-68$ & 1963 & June 8 & $\begin{array}{l}8.45 \\
8.74\end{array}$ & $\begin{array}{l}1,610 \\
1,750\end{array}$ & 6 \\
\hline 13 & $\begin{array}{l}\text { North Fork } \\
\text { Powder River } \\
\text { near Hazelton. }\end{array}$ & 24.5 & $\begin{array}{l}1947-68 \\
-\ldots \ldots\end{array}$ & 1953 & June 8 & $\begin{array}{r}54.34 \\
4.95\end{array}$ & $\begin{array}{l}886 \\
820\end{array}$ & $3 \overline{5}^{--}$ \\
\hline 14 & $\begin{array}{l}\text { North Fork } \\
\text { Powder River } \\
\text { near Mayo- } \\
\text { worth. }\end{array}$ & 106 & $1941-68$ & 1941 & June 9 & $\begin{array}{r}57.64 \\
6.90\end{array}$ & $\begin{array}{r}1,270 \\
952\end{array}$ & 12 \\
\hline 15 & $\begin{array}{l}\text { North Fork } \\
\text { Crazy Woman } \\
\text { Creek below } \\
\text { Spring Draw } \\
\text { near Buffalo. }\end{array}$ & 51.7 & $1950-68$ & 1966 & June 9 & $\begin{array}{l}5.99 \\
6.03\end{array}$ & $\begin{array}{l}1,150 \\
1,290\end{array}$ & 10 \\
\hline 16 & $\begin{array}{l}\text { North Fork } \\
\text { Crazy Woman } \\
\text { Creek near } \\
\text { Greub. }\end{array}$ & 174 & $1950-68$ & 1962 & June 8 & $\begin{array}{r}9.05 \\
11.29\end{array}$ & $\begin{array}{l}1,050 \\
1,640\end{array}$ & 6 \\
\hline 17 & $\begin{array}{l}\text { Middle Fork } \\
\text { Crazy Woman } \\
\text { Creek near } \\
\text { Greub. }\end{array}$ & 82.7 & $1942-68$ & $\begin{array}{l}1947 \\
-\cdots\end{array}$ & June 9 & $\begin{array}{r}5.77 \\
6.00\end{array}$ & $\begin{array}{l}4,520 \\
1,470\end{array}$ & 8 \\
\hline \multicolumn{9}{|c|}{ Green River basin } \\
\hline 18 & $\begin{array}{l}\text { Blacks Fork } \\
\text { near Milburne. }\end{array}$ & 156 & $1940-68$ & $\begin{array}{l}1957 \\
1965 \\
-\end{array}$ & June 20 & $\begin{array}{l}6.76 \\
6.07\end{array}$ & $\begin{array}{l}2,530 \\
1,940\end{array}$ & 4 \\
\hline 19 & $\begin{array}{l}\text { East Fork of } \\
\text { Smith Fork } \\
\text { near Robertson. }\end{array}$ & 53.0 & $\begin{array}{l}1940-68 \\
--1-\end{array}$ & 1965 & June 6 & $\begin{array}{l}6.75 \\
6.63\end{array}$ & $\begin{array}{l}1,450 \\
1,280\end{array}$ & $\overline{2} \overline{1}$ \\
\hline
\end{tabular}

See footnotes at end of table. 
TABLE 10.-Flood stages and discharges, June and August, in WyomingContinued

\begin{tabular}{|c|c|c|c|c|c|c|c|c|}
\hline \multirow{4}{*}{ No. } & \multirow{4}{*}{$\begin{array}{l}\text { Stream and place } \\
\text { of determination }\end{array}$} & \multirow{4}{*}{$\begin{array}{c}\text { Drainage } \\
\text { area } \\
\text { (sq mi) }\end{array}$} & \multicolumn{6}{|c|}{ Maximum floods } \\
\hline & & & \multirow{2}{*}{\multicolumn{2}{|c|}{$\begin{array}{c}\text { Known before } \\
\text { June } 1968\end{array}$}} & \multirow{3}{*}{$\begin{array}{l}\text { June, } \\
\text { August } \\
1968\end{array}$} & \multirow{3}{*}{$\begin{array}{c}\text { Gage } \\
\text { height } \\
\text { (feet) }\end{array}$} & \multicolumn{2}{|c|}{ Discharge } \\
\hline & & & & & & & \multirow[b]{2}{*}{ Cfs } & \multirow{2}{*}{$\begin{array}{c}\text { Recur- } \\
\text { rence } \\
\text { interval } \\
\text { (years) }\end{array}$} \\
\hline & & & Period & Year & & & & \\
\hline \multicolumn{9}{|c|}{ Green River basin-Continued } \\
\hline 20 & $\begin{array}{l}\text { West Fork of } \\
\text { Smith Fork } \\
\text { near Robert- } \\
\text { son. }\end{array}$ & 37.2 & $1940-68$ & $\begin{array}{c}1965 \\
-\ldots\end{array}$ & June 5 & $\begin{array}{r}63.20 \\
4.15\end{array}$ & $\begin{array}{l}2,100 \\
1,060\end{array}$ & $2 \overline{1.02}$ \\
\hline \multirow[t]{2}{*}{21} & \multirow{2}{*}{$\begin{array}{l}\text { Blacks Fork near } \\
\text { Lyman. }\end{array}$} & \multirow[t]{2}{*}{821} & \multirow{2}{*}{$\begin{array}{c}1938-57 \\
1962-68 . \\
\end{array}$} & 1965 & \multirow{2}{*}{ June 7} & 12.68 & 7,960 & -.. \\
\hline & & & & . - & & 10.47 & 4,000 & (4) \\
\hline 22 & $\begin{array}{c}\text { Blacks Fork } \\
\text { near Little } \\
\text { America. }\end{array}$ & 3,100 & $1962-68$ & $\begin{array}{r}1965 \\
\ldots\end{array}$ & June 8 & $\begin{array}{r}10.90 \\
8.43\end{array}$ & $\begin{array}{l}9,980 \\
4,380\end{array}$ & $\left({ }^{4}\right)$ \\
\hline 23 & $\begin{array}{c}\text { Summers Dry } \\
\text { Creek near } \\
\text { Green River. }\end{array}$ & 423 & $1965-68$ & 1965 & Aug. 11 & $\begin{array}{r}9.63 \\
10.73\end{array}$ & $\begin{array}{l}2,700 \\
3,830\end{array}$ & $(4)$ \\
\hline \multirow[t]{2}{*}{24} & \multirow{2}{*}{$\begin{array}{l}\text { Green River } \\
\text { tributary No. } 2 \\
\text { near Burntfork. }\end{array}$} & \multirow[t]{2}{*}{13.0} & \multirow{2}{*}{$\begin{array}{l}1959, \\
1961-68 .\end{array}$} & 1959 & \multirow{2}{*}{ Aug. 11} & 20.52 & 3,360 & \multirow{2}{*}{$\left({ }^{4}\right)$} \\
\hline & & & & $\ldots$ & & 7.97 & 490 & \\
\hline 25 & $\begin{array}{l}\text { Henrys Fork near } \\
\text { Lonetree. }\end{array}$ & 56 & $1943-68$ & 1965 & June 6 & $\begin{array}{l}5.79 \\
5.24\end{array}$ & $\begin{array}{l}2,010 \\
1,490\end{array}$ & 21.3 \\
\hline 26 & $\begin{array}{l}\text { Burnt Fork near } \\
\text { Burntfork. }\end{array}$ & 52.8 & $1943-68$ & 1965 & June 5 & $\begin{array}{l}\left({ }^{4}\right) \\
4.35\end{array}$ & $\begin{array}{r}3,200 \\
782\end{array}$ & 13 \\
\hline 27 & $\begin{array}{l}\text { Henrys Fork } \\
\text { tributary near } \\
\text { Manila, Utah. }\end{array}$ & 3.15 & $\begin{array}{l}1965-68 \\
-\end{array}$ & 1965 & Aug. 11 & $\begin{array}{l}5.81 \\
8.89\end{array}$ & ${ }^{(4)} 588$ & $(4)$ \\
\hline 28 & $\begin{array}{l}\text { Henrys Fork at } \\
\text { Linwood, Utah. }\end{array}$ & 520 & $\begin{array}{c}1929-68 \\
1929-58 \\
1960-68 .\end{array}$ & $\begin{array}{l}1959 \\
1936 \\
\ldots\end{array}$ & June 6 & $\begin{array}{r}59.42 \\
57.19 \\
6.51 \\
6.67\end{array}$ & $\begin{array}{l}\left({ }^{4}\right) \\
6,750 \\
1,960 \\
2,040\end{array}$ & 2 \\
\hline
\end{tabular}

1 Affected by backwater from ice.

2 Ratio of peak discharge to 50-year flood.

3 Affected by backwater from debris.

4 Not determined.

5 At different site and datum.

'Datum, 2.00 feet higher.

In Sweetwater County, Wyo., 0.8 miles north of Utah-Wyoming state line, 1.5 miles northwest of Linwood, Utah.

\section{FLOODS OF JUNE 23-28 IN CENTRAL AND EASTERN TEXAS}

By L. G. Stearns

On the afternoon of June 23, tropical storm Candy moved inland over the middle Texas coast. The storm weakened slowly as it moved north toward Dallas-Fort Worth metropolitan area. As a result of this storm, rainfall totals of 3 to 4 inches were common throughout central and eastern Texas, with numerous U.S. Weather Bureau stations recording 5 or more inches.

The heavy rains associated with tropical storm Candy followed 8 to 10 consecutive days of rain, thereby causing serious flooding along the middle and upper coastal regions (fig. 14).

Flooding in the San Jacinto River basin resulted in Lake Houston cresting at 47.52 feet on June 26, with flooding continuing through the end of June. The peak stage recorded at Lake Houston, 3 feet over the top of the spillway, was the second high- 


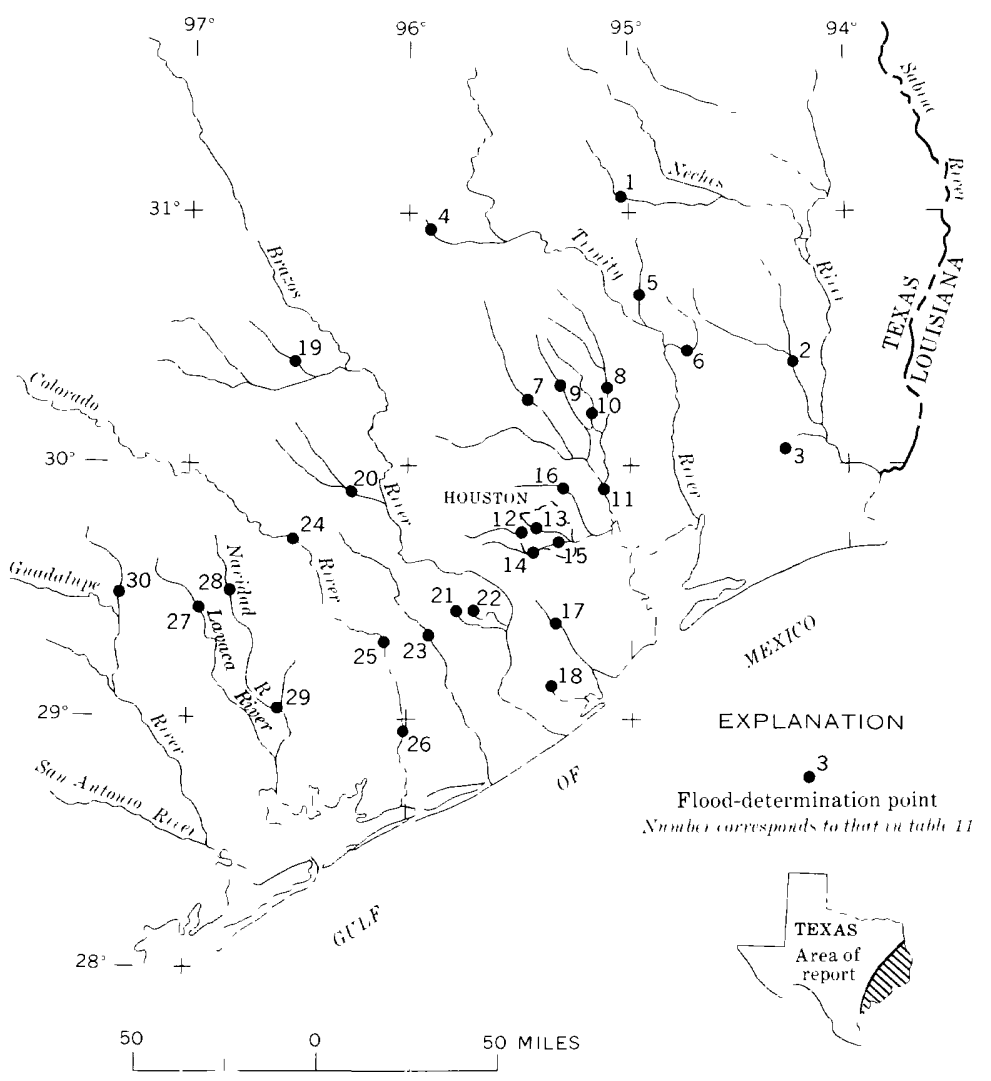

Figure 14.--Flood area; location of flood-determination points, floods of June $23-28$ in central and eastern Texas.

est stage of record and was surpassed only by the flood of September 12, 1961, which was a result of Hurricane Carla. No historical peak discharges were exceeded during this period; however, maximum peak discharges for the 1968 water year did occur at 30 stream-gaging stations in central and eastern Texas during the period June 23-28 (table 11).

No deaths or injuries resulted from this storm; however, estimates placed crop losses at $\$ 2,105,000$ and other property losses at $\$ 625,000$. 
TABLE 11.-Flood stages and discharges, June 23-28 in central and eastern Texas

\begin{tabular}{|c|c|c|c|c|c|c|c|c|}
\hline \multirow{4}{*}{ No. } & \multirow{4}{*}{$\begin{array}{l}\text { Stream and place } \\
\text { of determination }\end{array}$} & \multirow{4}{*}{$\begin{array}{c}\text { Drainage } \\
\text { area } \\
\text { (sq mi) }\end{array}$} & \multicolumn{6}{|c|}{ Maximum floods } \\
\hline & & & \multirow{2}{*}{\multicolumn{2}{|c|}{$\begin{array}{l}\text { Known before } \\
\text { June } 1968\end{array}$}} & \multirow{3}{*}{$\begin{array}{l}\text { June } \\
1968\end{array}$} & \multirow{3}{*}{$\begin{array}{l}\text { Gage } \\
\text { height } \\
\text { (feet) }\end{array}$} & \multicolumn{2}{|c|}{ Discharge } \\
\hline & & & & & & & \multirow[b]{2}{*}{ Cfs } & \multirow{2}{*}{$\begin{array}{c}\text { Recur- } \\
\text { rence } \\
\text { interval } \\
\text { (years) }\end{array}$} \\
\hline & & & Period & Year & & & & \\
\hline \multicolumn{9}{|c|}{ Neches River basin } \\
\hline 1 & $\begin{array}{l}\text { Piney Creek } \\
\text { near Groveton. }\end{array}$ & 79.0 & $\begin{array}{l}1921-68 \\
1962-68 \\
-\cdots---\end{array}$ & $\begin{array}{l}1942 \\
1966 \\
-\end{array}$ & $\overline{25}^{--}$ & $\begin{array}{l}17 \\
13.28 \\
14.53\end{array}$ & $\begin{array}{l}(1) \\
1,660 \\
4,280\end{array}$ & $-\cdots-$ \\
\hline 2 & $\begin{array}{l}\text { Village Creek } \\
\text { near Kountze. }\end{array}$ & 860 & $\begin{array}{c}1884-1968 \\
1924-27 \\
1939-68 .\end{array}$ & $\begin{array}{l}1915 \\
1940\end{array}$ & 26 & $\begin{array}{c}234 \\
27.6 \\
818.32\end{array}$ & $\begin{array}{l}(1) \\
67,200 \\
9,850\end{array}$ & 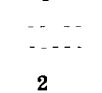 \\
\hline 3 & $\begin{array}{l}\text { Pine Island } \\
\text { Bayou near } \\
\text { Sour Lake. }\end{array}$ & 336 & $-\ldots$ & $-\cdots$ & 28 & 27.28 & 3,300 & 2 \\
\hline \multicolumn{9}{|c|}{ Trinity River basin } \\
\hline 4 & $\begin{array}{l}\text { Caney Creek } \\
\text { near Madison- } \\
\text { ville. }\end{array}$ & 112 & $\begin{array}{l}1900-68 \\
1963-68 \\
-\end{array}$ & $\begin{array}{l}1929 \\
1966 \\
-\end{array}$ & $24^{--}$ & $\begin{array}{l}22 \\
17.37 \\
17.32\end{array}$ & $\begin{array}{l}(1) \\
15,200 \\
11,800\end{array}$ & $18^{-\cdots}$ \\
\hline 5 & $\begin{array}{l}\text { Long King } \\
\text { Creek at } \\
\text { Livingston. }\end{array}$ & 141 & $\begin{array}{l}1870-1968 \\
1963-68 \\
\cdots\end{array}$ & $\begin{array}{l}1929 \\
1966 \\
----\end{array}$ & $24^{--}$ & $\begin{array}{l}41 \\
18.17 \\
17.40\end{array}$ & $\begin{array}{l}(1) \\
6,500 \\
6,700\end{array}$ & $-\overline{5}$ \\
\hline 6 & $\begin{array}{l}\text { Menard Creek } \\
\text { near Rye. }\end{array}$ & 152 & $\begin{array}{l}1961-68 \\
1966-68 \\
-\end{array}$ & $\begin{array}{l}1961 \\
1966 \\
---\end{array}$ & 26 & $\begin{array}{r}\mathbf{3 4} \\
+23.89 \\
20.74 \\
\end{array}$ & $\begin{array}{l}(1) \\
5,090 \\
1,810\end{array}$ & $2^{2}$ \\
\hline \multicolumn{9}{|c|}{ San Jacinto River basin } \\
\hline 7 & $\begin{array}{l}\text { West Fork San } \\
\text { Jacinto River } \\
\text { near Con roe. }\end{array}$ & 809 & $1913-68$ & 1940 & $\overline{\mathbf{2 5}}^{--}$ & $\begin{array}{l}30.85 \\
22.90\end{array}$ & $\begin{array}{r}110,000 \\
24,300\end{array}$ & $\overline{4}^{---}$ \\
\hline 8 & $\begin{array}{l}\text { East Fork San } \\
\text { Jacinto River } \\
\text { near Cleveland. }\end{array}$ & 325 & $1900-68$ & 1940 & $\overline{24}^{--}$ & $\begin{array}{r}520.37 \\
19.57\end{array}$ & $\begin{array}{l}59,000 \\
11,900\end{array}$ & $3^{-\cdots}$ \\
\hline 9 & $\begin{array}{l}\text { Caney Creek } \\
\text { near Splen- } \\
\text { dora. }\end{array}$ & 105 & $\begin{array}{l}1885-1968 \\
1944-68 \\
\cdots-\ldots\end{array}$ & $\begin{array}{l}1940 \\
1945 \\
\ldots\end{array}$ & $23^{--}$ & $\begin{array}{l}27 \\
23.19 \\
17.97\end{array}$ & $\begin{array}{r}(1) \\
14,900 \\
3,670\end{array}$ & $\overline{3}^{-\cdots}$ \\
\hline 10 & $\begin{array}{l}\text { Peach Creek } \\
\text { at Splendora. }\end{array}$ & 117 & $1895-1968$ & 1949 & $\overline{25}^{--}$ & $\begin{array}{l}22.73 \\
17.00\end{array}$ & $\begin{array}{r}28,500 \\
4,200\end{array}$ & $-\overline{3}$ \\
\hline 11 & $\begin{array}{l}\text { Lake Houston } \\
\text { near Sheldon. }\end{array}$ & 2,828 & $1954-68$ & 1961 & 26 & $\begin{array}{l}47.87 \\
47.52\end{array}$ & $\begin{array}{l}6205,000 \\
6187,100\end{array}$ & $-\cdots$ \\
\hline 12 & $\begin{array}{c}\text { Keegans Bayou } \\
\text { at Roark } \\
\text { Road near } \\
\text { Houston. }\end{array}$ & 9.28 & $1965-68$ & 1966 & $\overline{23}^{--}$ & $\begin{array}{l}67.64 \\
67.89\end{array}$ & $\begin{array}{l}588 \\
352\end{array}$ & $\cdots$ \\
\hline 13 & $\begin{array}{l}\text { Brays Bayou } \\
\text { at Houston. }\end{array}$ & 88.4 & $1911-68$ & $\begin{array}{l}1919 \\
1960 \\
-\end{array}$ & $\overline{23}^{--}$ & $\begin{array}{l}56.0 \\
49.72 \\
40.48\end{array}$ & $\begin{array}{l}(1) \\
12,600 \\
12,000\end{array}$ & $2 \overline{8}^{-\cdots}$ \\
\hline 14 & $\begin{array}{l}\text { Sims Bayou } \\
\text { at Hiram } \\
\text { Clarke Street, } \\
\text { Houston. }\end{array}$ & 20.2 & $1965-68$ & 1966 & $\overline{23}^{--}$ & $\begin{array}{l}\mathbf{5 1 . 0 8} \\
\mathbf{5 2 . 3 5}\end{array}$ & $\begin{array}{l}2,280 \\
2,200\end{array}$ & $12^{---}$ \\
\hline 15 & $\begin{array}{l}\text { Sims Bayou } \\
\text { at Houston. }\end{array}$ & 64.0 & $1953-68$ & 1960 & $\overline{24}^{--}$ & $\begin{array}{l}29.76 \\
24.80\end{array}$ & $\begin{array}{l}8,030 \\
4,680\end{array}$ & 12 \\
\hline 16 & $\begin{array}{l}\text { Greens Bayou } \\
\text { near Houston. }\end{array}$ & 72.7 & $\begin{array}{l}1953-68 \\
-\ldots-1 .\end{array}$ & $\begin{array}{l}1954 \\
1961 \\
-\end{array}$ & $\overline{24}^{-\cdots}$ & $\begin{array}{l}65.75 \\
62.82\end{array}$ & $\begin{array}{r}7,000 \\
2,580\end{array}$ & $\mathbf{2}$ \\
\hline \multicolumn{9}{|c|}{ Chocolate Bayou basin } \\
\hline 17 & $\begin{array}{l}\text { Chocolate Bayou } \\
\text { near Alvin. }\end{array}$ & 87.7 & $1947-68$ & 1949 & 24 & $\begin{array}{r}81.80 \\
20.34\end{array}$ & $\begin{array}{l}7,400 \\
4,160\end{array}$ & $2^{\cdots}$ \\
\hline \multicolumn{9}{|c|}{ Oyster Creek basin } \\
\hline 18 & $\begin{array}{l}\text { Oyster Creek } \\
\text { near Angleton. }\end{array}$ & 211 & $\begin{array}{l}1900-68 \\
1945-68 \\
-\cdots---\end{array}$ & $\begin{array}{l}1913 \\
1957 \\
---\end{array}$ & $\overrightarrow{26}$ & $\begin{array}{l}32.2 \\
31.45 \\
27.78\end{array}$ & $\begin{array}{c}\left({ }^{1}\right) \\
10,600 \\
3,080\end{array}$ & $\overline{2}^{-\cdots}$ \\
\hline
\end{tabular}

See footnotes at end of table. 
TABLE 11.-Flood stages and discharges, June 23-28 in central and eastern Texas-Continued

\begin{tabular}{|c|c|c|c|c|c|c|c|c|}
\hline \multirow{4}{*}{ No. } & \multirow{4}{*}{$\begin{array}{l}\text { Stream and place } \\
\text { of determination }\end{array}$} & \multirow{4}{*}{$\begin{array}{c}\text { Drainage } \\
\text { area } \\
\text { (sq mi) }\end{array}$} & \multicolumn{6}{|c|}{ Maximum floods } \\
\hline & & & \multirow{2}{*}{\multicolumn{2}{|c|}{$\begin{array}{c}\text { Known before } \\
\text { June } 1968\end{array}$}} & \multirow{3}{*}{$\begin{array}{l}\text { June } \\
1968\end{array}$} & \multirow{3}{*}{$\begin{array}{l}\text { Gage } \\
\text { height } \\
\text { (feet) }\end{array}$} & \multicolumn{2}{|c|}{ Discharge } \\
\hline & & & & & & & & Recur- \\
\hline & & & Period & Year & & & Cfs & $\begin{array}{c}\text { interval } \\
\text { (years) }\end{array}$ \\
\hline \multicolumn{9}{|c|}{ Brazos River basin } \\
\hline 19 & $\begin{array}{l}\text { Davidson } \\
\text { Creek near } \\
\text { Lyons. }\end{array}$ & 195 & $\begin{array}{l}1902-68 \\
1962-68 \\
-\ldots\end{array}$ & $\begin{array}{l}1947 \\
1962 \\
-\ldots\end{array}$ & $24^{--}$ & $\begin{array}{l}17 \\
16.08 \\
18.67\end{array}$ & $\begin{array}{c}\left({ }^{1}\right) \\
6,000 \\
23,200\end{array}$ & $\overline{34}$ \\
\hline 20 & $\begin{array}{l}\text { Mill Creek near } \\
\text { Bellville. }\end{array}$ & 377 & $\begin{array}{l}1899-1968 \\
1963-68 \\
-\ldots-\ldots\end{array}$ & $\begin{array}{l}1940 \\
1965 \\
-\cdots\end{array}$ & $25^{--}$ & $\begin{array}{l}22.8 \\
15.20 \\
15.98\end{array}$ & $\begin{array}{c}(1) \\
26,200 \\
34,000\end{array}$ & $\overline{34}$ \\
\hline 21 & $\begin{array}{l}\text { Big Creek near } \\
\text { Needville. }\end{array}$ & 42.3 & $\begin{array}{l}1913-68 \\
1947-68 \\
\cdots\end{array}$ & $\begin{array}{c}1945 \\
1960 \\
--\end{array}$ & 23 or 24 & $\begin{array}{l}824.4 \\
823.81 \\
20.71\end{array}$ & $\begin{array}{r}\left.{ }^{1}\right) \\
10,400 \\
3,400\end{array}$ & $1 \overline{4}$ \\
\hline 22 & $\begin{array}{l}\text { Dry Creek near } \\
\text { Rosenberg. }\end{array}$ & 8.53 & $1932-68$ & $\begin{array}{l}1959 \\
--\end{array}$ & $\overline{24}$ & $\begin{array}{l}12.66 \\
10.30\end{array}$ & $\begin{array}{r}2,410 \\
860 \\
\end{array}$ & (1) \\
\hline \multicolumn{9}{|c|}{ San Bernard River basin } \\
\hline 23 & $\begin{array}{l}\text { San Bernard } \\
\text { River near } \\
\text { Boling. }\end{array}$ & 727 & $\begin{array}{l}1900-68 \\
1954-68 \\
-\end{array}$ & $\begin{array}{l}1913 \\
1960 \\
-\end{array}$ & 27 & $\begin{array}{l}43.5 \\
42.41 \\
36.75\end{array}$ & $\begin{array}{c}\left({ }^{(}\right) \\
21,200 \\
13,200\end{array}$ & $-\cdots$ \\
\hline \multicolumn{9}{|c|}{ Colorado River basin } \\
\hline 24 & $\begin{array}{l}\text { Colorado River } \\
\text { at Columbus. }\end{array}$ & 1041,070 & $\begin{array}{l}1852-1968 \\
1916-68 \\
\ldots \ldots\end{array}$ & $\begin{array}{l}1869 \\
1913 \\
1935 \\
-\end{array}$ & $\overline{25}^{\cdots}$ & $\begin{array}{l}41.6 \\
41.6 \\
38.5 \\
24.16\end{array}$ & $\begin{array}{c}(1) \\
(1) \\
190,000 \\
49,800\end{array}$ & 6 \\
\hline \multirow[t]{2}{*}{25} & $\begin{array}{l}\text { Colorado River } \\
\text { at Wharton. }\end{array}$ & in 41,380 & $\begin{array}{l}1869-1968 \\
1919-25 \\
1929,1935, \\
1938-68 .\end{array}$ & $\begin{array}{l}1869 \\
1913 \\
1935\end{array}$ & $\begin{array}{l}-- \\
\cdots- \\
\cdots-\end{array}$ & $\begin{array}{l}38.9 \\
38.9 \\
38.2\end{array}$ & $\begin{array}{c}\left(\begin{array}{c}(1) \\
(1) \\
159,000\end{array}\right.\end{array}$ & - \\
\hline & & & 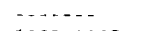 & $\ldots$ & 26 & 28.06 & 55,400 & 8 \\
\hline \multirow[t]{3}{*}{26} & $\begin{array}{l}\text { Colorado River } \\
\text { near Bay City. }\end{array}$ & 1041,650 & $\begin{array}{l}1869-1968 \\
1940 \\
1948-68 .\end{array}$ & $\begin{array}{l}1869 \\
1913 \\
1960\end{array}$ & $\begin{array}{l}\cdots \\
\cdots \\
\cdots\end{array}$ & $\begin{array}{l}56.1 \\
56.1 \\
48.2\end{array}$ & $\begin{array}{l}(1) \\
(1) \\
84,100\end{array}$ & $\begin{array}{c}- \\
\cdots \\
\cdots\end{array}$ \\
\hline & & & 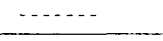 & $-\cdots$ & 26 & 37.49 & 49,500 & 6 \\
\hline & \multicolumn{8}{|c|}{ Lavaca River basin } \\
\hline 27 & $\begin{array}{c}\text { Lavaca River at } \\
\text { Hallettsville. }\end{array}$ & 101 & $1840-68$ & 1940 & $24^{--}$ & $\begin{array}{l}40.60 \\
24.44\end{array}$ & $\begin{array}{r}93,100 \\
6,590\end{array}$ & $3^{-\cdots}$ \\
\hline 28 & $\begin{array}{l}\text { Navidad River } \\
\text { near } \\
\text { Hallettsville. }\end{array}$ & 333 & $\begin{array}{l}1860-1968 \\
1962-68 \\
-\end{array}$ & $\begin{array}{l}1940 \\
1967 \\
---\end{array}$ & $24^{\cdots}$ & $\begin{array}{l}40 \\
30.34 \\
28.28\end{array}$ & $\begin{array}{l}(1) \\
19,500 \\
12,800\end{array}$ & $\overline{3}^{-}$ \\
\hline 29 & $\begin{array}{c}\text { Navidad River } \\
\text { near Ganado. }\end{array}$ & 1,116 & $\begin{array}{l}1876-1968 \\
-\end{array}$ & 1936 & 26 & $\begin{array}{l}39.8 \\
\mathbf{3 3 . 1 5}\end{array}$ & $\begin{array}{l}94,000 \\
32,200\end{array}$ & 6 \\
\hline \multicolumn{9}{|c|}{ Guadalupe River basin } \\
\hline 30 & $\begin{array}{l}\text { Peach Creek } \\
\text { below } \\
\text { Dilworth. }\end{array}$ & 462 & $\begin{array}{l}1840-1968 \\
1960-68 \\
\cdots\end{array}$ & $\begin{array}{l}1940 \\
1960 \\
-\end{array}$ & $\overline{25}$ & $\begin{array}{l}35.3 \\
31.28 \\
28.86\end{array}$ & $\begin{array}{c}(1) \\
23,800 \\
10,400\end{array}$ & $2^{\cdots}$ \\
\hline
\end{tabular}

1 Not determined.

${ }^{2}$ At site 2,000 feet downstream at same datum.

${ }^{3}$ At different site and datum.

4 Result of dam failure.

"At site 1,800 feet upstream and datum 5,000 feet higher.

" Contents in acre-feet.

i At former site before channel rectification.

${ }^{8}$ Before channel rectification.

${ }^{9}$ Includes overflow from Brazos River.

${ }^{10}$ Of which $11,900 \mathrm{sq} \mathrm{mi}$ is probably noncontributing. 


\section{FLOODS OF JUNE 24 IN CENTRAL NEBRASKA}

By H. D. Brice

Widespread rain fell on June $23-24$ in central Nebraska. The larger amounts recorded were 5 to 6 inches in the upper reach of Wood River and 7 to 10 inches in the lower reach of South Loup River (fig. 15, table 12). A rancher living 1 mile east of Rockville reported that he measured 3.6 inches of rain in 1 hour, a pointrainfall rate having a recurrence interval of about 100 years (U.S. Weather Bureau, 1961). Hail and high-velocity wind accompaning the rain caused considerable damage.

Runoff from the storm caused Wood River to overflow in the vicinity of Lomax and South Loup River to overflow from above Ravenna to below St. Michael. The peak discharge near Lomax, $1,270 \mathrm{cfs}$, was the third largest in the 17 years of record and its recurrence interval is estimated to be 40 years (table 13). The peak discharges at Ravenna and St. Michael were greater than those for a 50-year flood.

Because the approaches to bridges were washed out and electric power and telephone service were cut off, Ravenna was almost completely isolated for a time. Wind, hail, and flood damages from the storm were estimated at $\$ 1,260,000$.

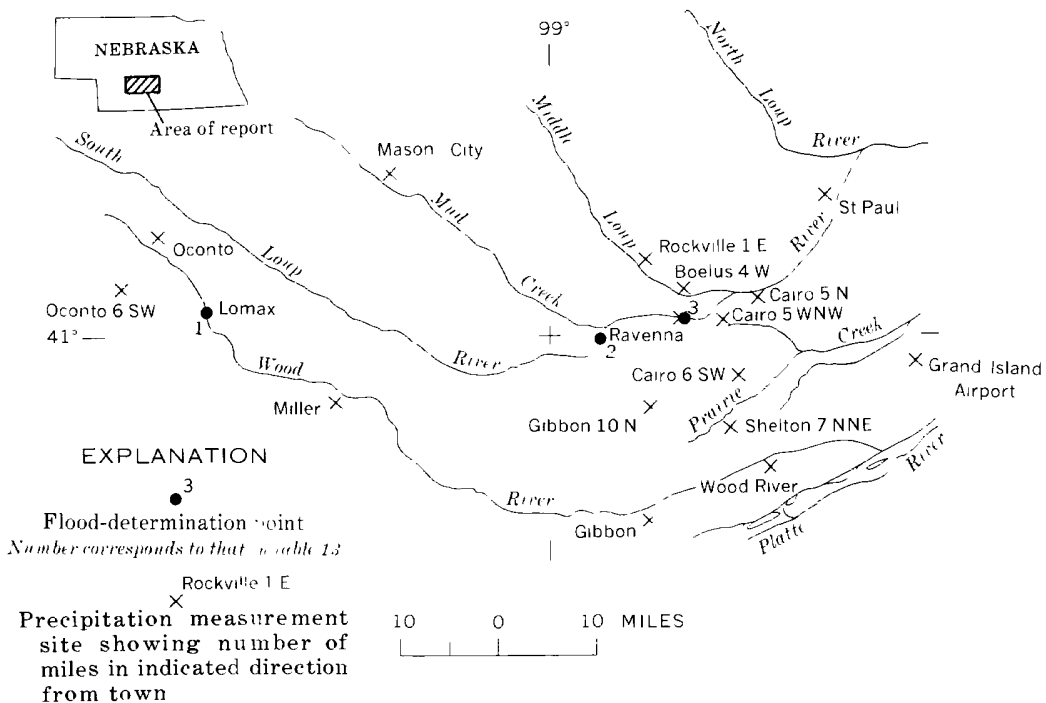

FIGURE 15.--Floorl area; location of flood-determination points and rainfall data sites, floods of June 24 in central Nebraska. 
TABLE 12.-Daily rainfall, in inches, associated with floods of June 24 in central Nebraska

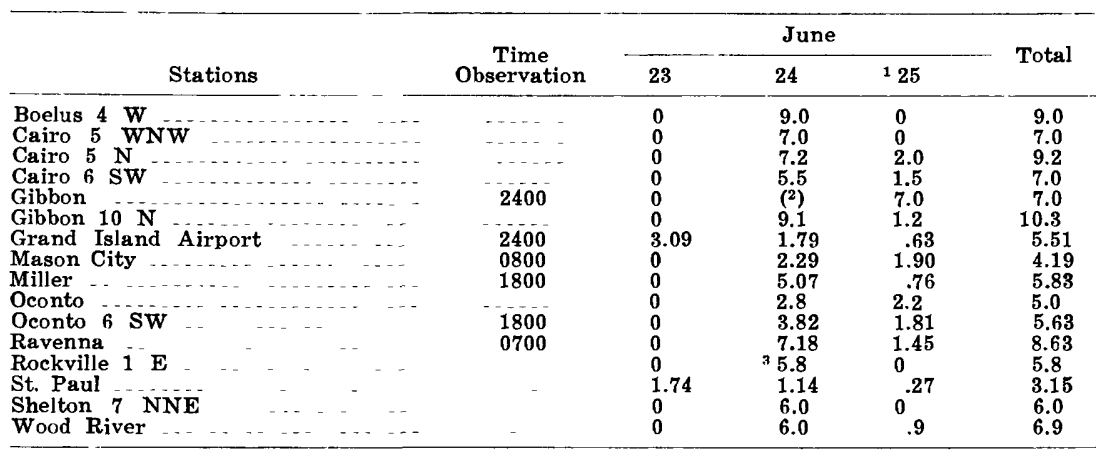

1 Readings for this day include rain that fell during part of the precering day.

2 Included in amount for following day.

${ }^{3}$ Of which 3.6 inches fell in 1 hour.

TABLE 13.-Flood stages and discharges, June 24, Platte River basin, central. Nebraska

\begin{tabular}{|c|c|c|c|c|c|c|c|c|}
\hline \multirow{4}{*}{ No. } & \multirow{4}{*}{$\begin{array}{l}\text { Stream and place } \\
\text { of determination }\end{array}$} & \multirow{4}{*}{$\begin{array}{l}\text { Drainage } \\
\text { area } \\
\text { (sq mi) }\end{array}$} & \multicolumn{6}{|c|}{ Maximum floods } \\
\hline & & & \multirow{2}{*}{\multicolumn{2}{|c|}{$\begin{array}{l}\text { Known before } \\
\text { June } 1968\end{array}$}} & \multirow{3}{*}{$\begin{array}{l}\text { June } \\
1968\end{array}$} & \multirow{3}{*}{$\begin{array}{l}\text { Gage } \\
\text { height } \\
\text { (feet) }\end{array}$} & \multicolumn{2}{|c|}{ Discharge } \\
\hline & & & & & & & & Recur- \\
\hline & & & Period & Year & & & Cfs & $\begin{array}{c}\text { interva } \\
\text { (years) }\end{array}$ \\
\hline 1 & $\begin{array}{l}\text { Wood River near } \\
\text { Lomax. }\end{array}$ & 79.6 & $1952-68$ & 1960 & 24 & $18.82^{-}$ & $\begin{array}{l}1,750 \\
1,270\end{array}$ & 40 \\
\hline 2 & $\begin{array}{l}\text { South Loup River } \\
\text { at Ravenna. }\end{array}$ & $\begin{array}{l}1,660 \\
2890\end{array}$ & $\begin{array}{r}1941-58 . \\
1968 .\end{array}$ & 1947 & & 12.6 & (1) & \\
\hline 3 & $\begin{array}{l}\text { South Loup River } \\
\text { at St. Michael. }\end{array}$ & $\begin{array}{r}2,560 \\
21,650\end{array}$ & $1943-68$ & 1947 & $\begin{array}{l}24 \\
24\end{array}$ & $\begin{array}{l}10.32 \\
(1) \\
11.00\end{array}$ & $\begin{array}{c}17,100 \\
(1) \\
27,500\end{array}$ & $\begin{array}{l}31.07 \\
31.25\end{array}$ \\
\hline
\end{tabular}

1 Not determined.

2 Contributing area.

${ }^{3}$ Ratio of peak discharge to 50 -year flood.

\section{FLOOD OF JULY 9 IN CANYON CREEK BASIN NEAR LOWMAN, IDAHO}

\section{By C. A. Thomas}

Intense flooding occurred in a few square miles of mountainous terrain along Canyon Creek near Lowman, Idaho (fig. 16), from precipitation associated with a thunderstorm of short duration which began about 6:30 p.m. on July 9. No precipitation data were available for the flood area, and only scattered light showers were recorded at nearby weather stations.

The resulting flood, although confined to a small area in a sparsely settled area, was outstanding owing to the unusually high unit discharges. An indirect measurement of the peak discharge in a small tributary to Canyon Creek determined that a discharge of $1,550 \mathrm{cfs}$ was produced from a drainage area of only one-fourth of a square mile. The high flow lasted for about 1 


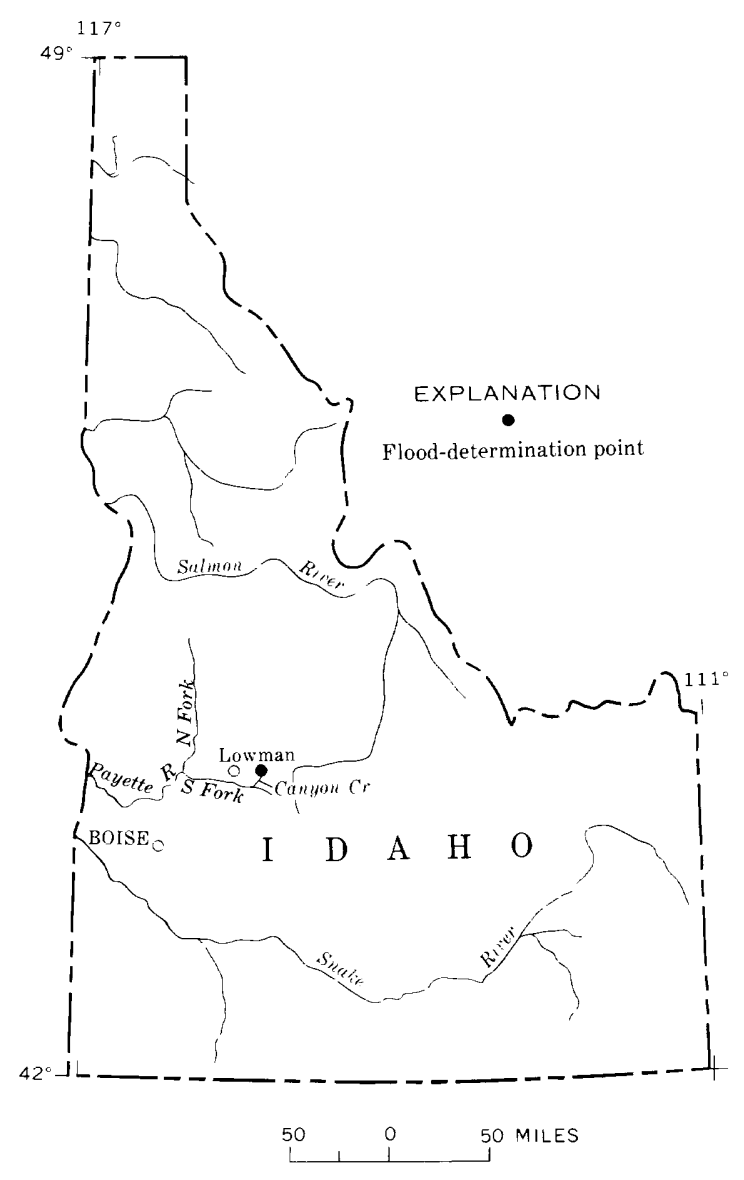

FiguRE 16.-Flood area; location of flood-determination point, flood of July 9 in Canyon Creek basin near Lowman, Idaho.

hour, and about $1 \mathrm{cfs}$ was still flowing in the ephemeral channel on July 12, a notably long period for such a flow to continue.

The stream channel was severely eroded, and debris and gravel were deposited up to 6 feet deep on adjacent State Highway 21 .

\section{FLOODS OF JULY 16-20 IN NORTHEASTERN IOWA}

By HARLAN H. Schwob

A severe storm occurred in the central part of northeastern Iowa on the night of July 16-17. The storm caused record floods 
in the central part of the Wapsipinicon River basin and on tributaries of the Cedar River, in the Iowa River basin. Four lives were lost, three by drowning and one by electrocution. A floodcontrol dam on Virden Creek was overtopped and the outlet structure was washed out when the design flood was exceeded. Total damage was estimated at $\$ 14.5$ million.

Three areas had total rainfall of 14 or more inches during the storm. As shown in figure 17, these three areas were at Waverly, north of Waterloo, and north of Independence. The official amount of 12.40 inches in an observational day (July 17) at the Shell Rock rain gage was a record for northeastern Iowa. An unofficial 16.20 inches in Waverly, from a bucket survey, was the greatest 24-hour rainfall known in northeastern Iowa (U.S. Weather Bureau, 1968). Three recording rain gages were in the storm area although not in any of the specific areas of greatest total fall. Figure 18 is a graph of the accumulated rainfall at the gages for July 16 and 17 . The graph shows that most of the rainfall was during a 12-hour nighttime period at the three widely separated gages.

Flood-peak data (table 14) were obtained at complete-record gaging stations, partial-record gaging stations, and at miscellaneous sites (fig. 17).

The Wapsipinicon River flood at the Independence gage (No. 5 , table 14) was maximum for the period of record. The stage of 21.11 feet is the greatest known since at least 1901. Only about half of the drainage area of 1,048 square miles at the gage received 4 or more inches of rain. The tributaries of the Wapsipinicon River, principally the Little Wapsipinicon River and Crane Creek, were discharging extremely large amounts of water. Smaller, unmeasured tributaries were also contributing large amounts of water to the flooding river upstream from Independence.

The heavy rains near Independence produced record floods on Malone Creek at Independence (No. 6, table 14) and on Pine and Buffalo Creeks, 6 and 10 miles, respectively, east of Independence. These streams are tributary to the Wapsipinicon River downstream from the Independence gage. As the crests on these streams and on the Wapsipinicon River moved downstream, the peak discharge was attenuated by valley storage and the lack of sustaining flow from the lower tributaries. Near the mouth of Wapsipinicon River (No. 14, table 14) the peak discharge was that of a flood with a recurrence interval of only 2 years. 


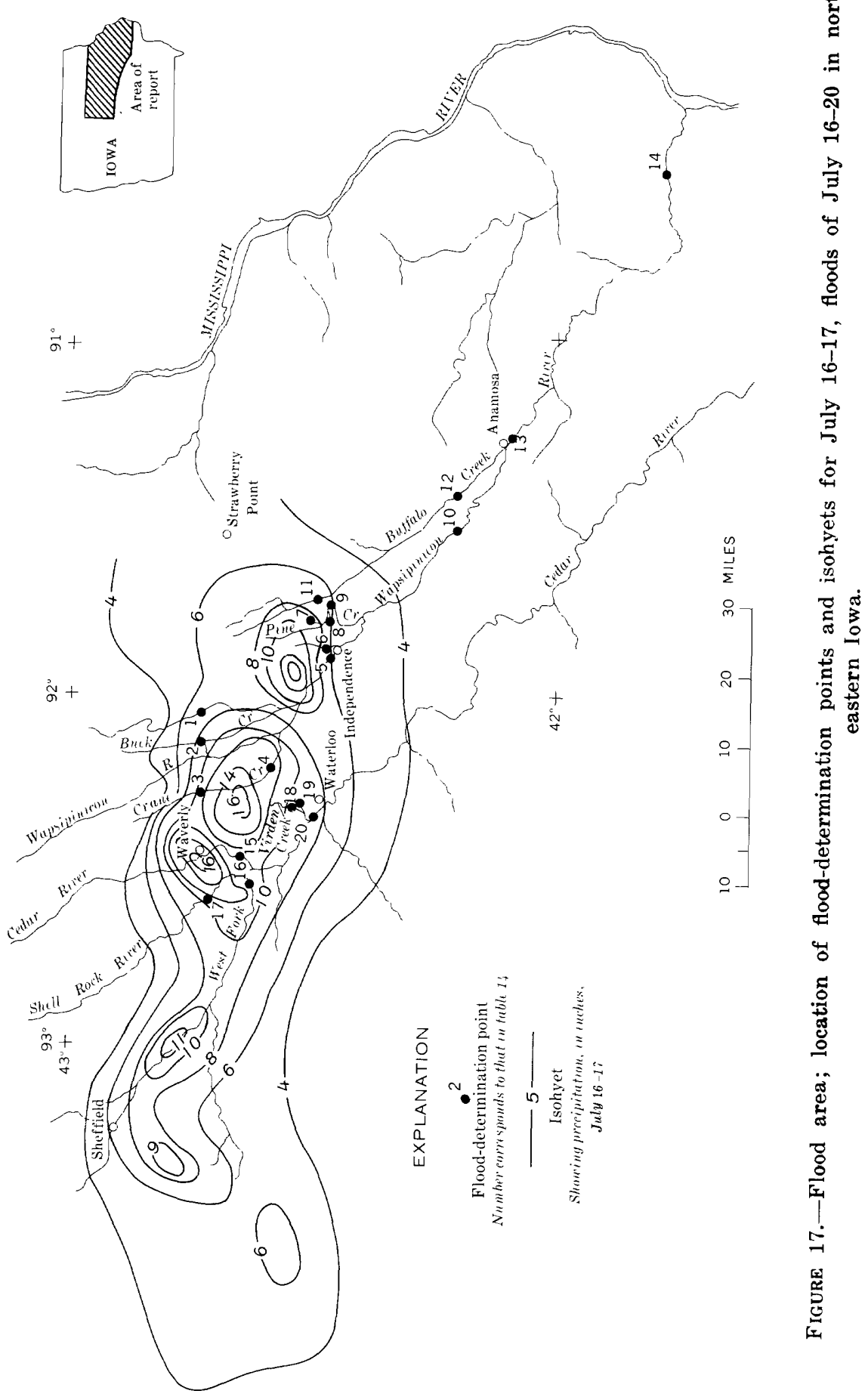




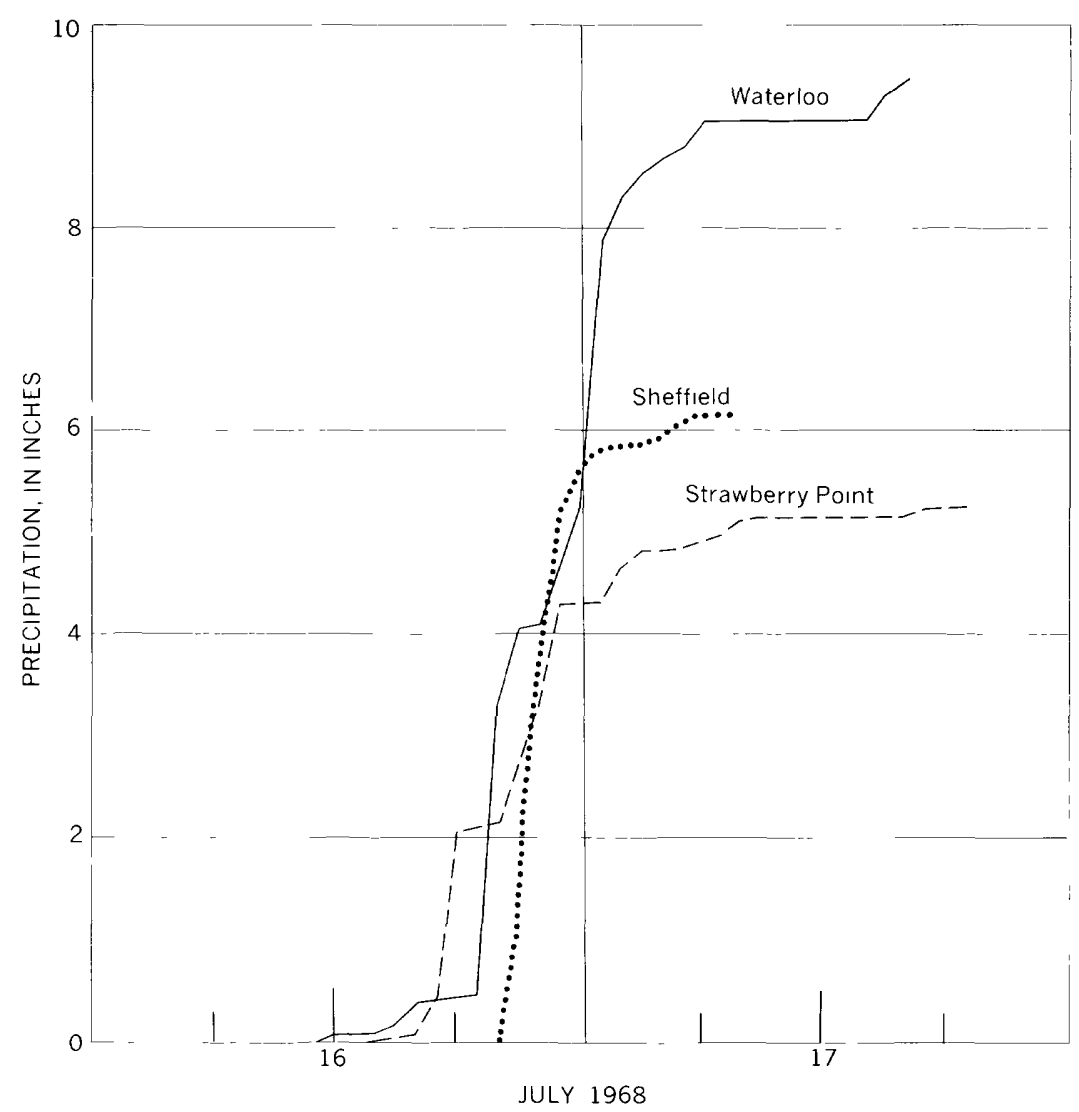

FIgURE 18.-Accumulated precipitation at three stations, floods of July 16-20 northeastern Iowa.

A relatively small part of the Cedar River basin was in the storm area. Consequently, floods on the major tributaries and the main stem were minor-generally less than a 10 -year flood. However, smaller tributaries in the vicinity of Waterloo, notably Virden Creek, produced large and damaging floods. Two miscellaneous measurements (Nos. 18, 19, table 14) indicate the severity of the floods on this creek.

The Corps of Engineers determined the damages or costs caused by the flood in three cities. Floods on Malone Creek caused $\$ 93,000$ damage at Independence, and floods on Virden Creek caused $\$ 950,000$ damage at Waterloo. On Wapsipinicon River flood fighting at Anamosa reportedly cost $\$ 20,000$. 
TABLE 14.-Flood stages and discharges, July 16-20 in northeastern Iowa

\begin{tabular}{|c|c|c|c|c|c|c|c|c|}
\hline \multirow{4}{*}{ No. } & \multirow{4}{*}{$\begin{array}{l}\text { Stream and place } \\
\text { of determination }\end{array}$} & \multirow{4}{*}{$\begin{array}{l}\text { Drainage } \\
\text { area } \\
\text { (sq mi) }\end{array}$} & \multicolumn{6}{|c|}{ Maximum floods } \\
\hline & & & \multirow{2}{*}{\multicolumn{2}{|c|}{$\begin{array}{l}\text { Known before } \\
\text { July } 1968\end{array}$}} & \multirow{3}{*}{$\begin{array}{l}\text { July } \\
1968\end{array}$} & \multirow{3}{*}{$\begin{array}{l}\text { Gage } \\
\text { height } \\
\text { (feet) }\end{array}$} & \multirow[b]{3}{*}{ Cfs } & ge \\
\hline & & & & & & & & \multirow{2}{*}{$\begin{array}{l}\text { Recur- } \\
\text { rence } \\
\text { interval } \\
\text { (years) }\end{array}$} \\
\hline & & & Period & Year & & & & \\
\hline \multicolumn{9}{|c|}{ Wapsipinicon River basin } \\
\hline 1 & $\begin{array}{l}\text { Little Wapsipini- } \\
\text { con River } \\
\text { near Oran. }\end{array}$ & 94.1 & $1966-68$ & 1966 & 17 & $\begin{array}{l}87.75 \\
90.00\end{array}$ & $\begin{array}{l}1,450 \\
5,000\end{array}$ & 1.27 \\
\hline 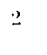 & $\begin{array}{l}\text { Buck Creek near } \\
\text { Oran. }\end{array}$ & 37.9 & $1966-68$ & 1966 & 17 & $\begin{array}{l}88.94 \\
89.11\end{array}$ & $\begin{array}{l}800 \\
950\end{array}$ & $<2$ \\
\hline 3 & $\begin{array}{l}\text { Crane Creek } \\
\text { near Readlyn. }\end{array}$ & 45.5 & - & $\ldots$ & 17 & $=988.78$ & 2,750 & 35 \\
\hline 4 & $\begin{array}{l}\text { Crane Creek } \\
\text { near Dunker- } \\
\text { ton. }\end{array}$ & 82.3 & $\ldots$ & - & 17 & 2959.84 & 17,000 & ${ }^{1} 5.05$ \\
\hline 5 & $\begin{array}{l}\text { Wapsipinicon } \\
\text { River at } \\
\text { Independence. }\end{array}$ & 1,048 & $\begin{array}{c}1934-68 \\
--\end{array}$ & 1947 & 18 & $\begin{array}{l}18.74 \\
21.11\end{array}$ & $\begin{array}{l}21,500 \\
26,800\end{array}$ & ${ }^{1} 1.06$ \\
\hline 6 & $\begin{array}{l}\text { Malone Creek at } \\
\text { Independence. }\end{array}$ & 10.0 & 1950 & 1950 & 17 & $\ldots$ & $\begin{array}{r}4,910 \\
10,700\end{array}$ & $>50$ \\
\hline 7 & $\begin{array}{l}\text { Pine Creek } \\
\text { tributary near } \\
\text { Winthrop. }\end{array}$ & .33 & $\begin{array}{l}1953-68 \\
\ldots\end{array}$ & $\begin{array}{l}1959 \\
\cdots\end{array}$ & 17 & $\begin{array}{l}8.67 \\
8.97\end{array}$ & $\begin{array}{l}304 \\
334\end{array}$ & 29 \\
\hline 8 & $\begin{array}{l}\text { Pine Creek near } \\
\text { Winthrop. }\end{array}$ & 28.3 & $\begin{array}{l}1950-68 \\
-\end{array}$ & 1950 & 17 & $\begin{array}{l}21.70 \\
22.98\end{array}$ & $\begin{array}{l}14,500 \\
24.200\end{array}$ & ${ }^{-} 6.35$ \\
\hline 9 & $\begin{array}{l}\text { Pine Creek } \\
\text { tributary at } \\
\text { Winthrop. }\end{array}$ & .70 & $\begin{array}{l}1953-68 \\
\ldots\end{array}$ & 1959 & 17 & $\begin{array}{l}7.12 \\
7.26\end{array}$ & $\begin{array}{l}443 \\
570\end{array}$ & 11.04 \\
\hline 10 & $\begin{array}{l}\text { Wapsipinicon } \\
\text { River at } \\
\text { Central City. }\end{array}$ & 1,263 & $\begin{array}{l}1929 \\
1941-50\end{array}$ & 1947 & 19 & $\begin{array}{l}2835.24 \\
=829.53\end{array}$ & $\begin{array}{l}22,500 \\
23,000\end{array}$ & 20 \\
\hline 11 & $\begin{array}{l}\text { Buffalo Creek } \\
\text { near Winthrop. }\end{array}$ & 68.2 & $1957-68$ & 1960 & 17 & $\begin{array}{l}18.59 \\
19.36\end{array}$ & $\begin{array}{r}5,350 \\
14,100\end{array}$ & ${ }^{1} 2.33$ \\
\hline 12 & $\begin{array}{l}\text { Buffalo Creek } \\
\text { near Central } \\
\text { City. }\end{array}$ & 185 & 1964 & 1964 & 18 & $\begin{array}{l}2847.84 \\
2848.58\end{array}$ & $\begin{array}{l}3,600 \\
4,000\end{array}$ & 3 \\
\hline 13 & $\begin{array}{l}\text { Wapsipinicon } \\
\text { River near } \\
\text { Anamosa. }\end{array}$ & 1,575 & $\begin{array}{l}1962,63 \\
\ldots \ldots\end{array}$ & 1962 & 20 & $\begin{array}{l}2775.61 \\
2777.90\end{array}$ & $\begin{array}{l}15,400 \\
19,000\end{array}$ & 8 \\
\hline 14 & $\begin{array}{l}\text { Wapsipinicon } \\
\text { River near } \\
\text { DeWitt. }\end{array}$ & 2,330 & $1935-68$ & 1944 & 25 & $\begin{array}{l}12.07 \\
11.91\end{array}$ & $\begin{array}{l}26,000 \\
13,800\end{array}$ & 2 \\
\hline \multicolumn{9}{|c|}{ Iowa River basin } \\
\hline 15 & $\begin{array}{l}\text { Cedar River at } \\
\text { Janesville. }\end{array}$ & 1,661 & $\begin{array}{c}1905-06, \\
1915-21, \\
1923-27, \\
1929, \\
1933-42 \\
1945-68 .\end{array}$ & 1961 & $\ldots$ & 16.33 & 37,000 & - \\
\hline & & & & & 17 & 12.79 & 21,700 & 6 \\
\hline 16 & $\begin{array}{l}\text { West Fork Cedar } \\
\text { River at } \\
\text { Finchford. }\end{array}$ & 846 & $1945-68$ & 1951 & 19 & $\begin{array}{l}17.28 \\
14.51\end{array}$ & $\begin{array}{l}31,900 \\
10,600\end{array}$ & 3 \\
\hline 17 & $\begin{array}{l}\text { Shell Rock River } \\
\text { at Shell Rock. }\end{array}$ & 1,746 & $\begin{array}{l}1856 \\
1953-68 .\end{array}$ & 1856 & $\cdots$ & 17.7 & 45,000 & $\cdots$ \\
\hline & & & $\ldots . .$. & $\ldots$ & 17 & 10.68 & 4,040 & $<2$ \\
\hline 18 & $\begin{array}{l}\text { Virden Creek near } \\
\text { Waterloo. }\end{array}$ & 8.64 & $\cdots$ & $\ldots$ & 17 & $\cdots$ & 6,640 & $>50$ \\
\hline 19 & $\begin{array}{l}\text { Virden Creek } \\
\text { tributary at } \\
\text { Waterloo. }\end{array}$ & .58 & $\ldots .$. & $\ldots$ & 17 & $\ldots$. & 811 & $>50$ \\
\hline 20 & $\begin{array}{l}\text { Cedar River at } \\
\text { Waterloo. }\end{array}$ & 5,146 & $\begin{array}{l}1929 \\
1933 \\
1941-68 .\end{array}$ & 1961 & $\ldots$ & 21.86 & 76,700 & -. \\
\hline & & & & - & 18 & 12.35 & 22,800 & 2 \\
\hline
\end{tabular}

${ }^{1}$ Ratio of peak discharge to 50 -year flood.

2 Elevation in feet above mean sea level (1929 adjustment). 


\section{FLOODS OF JULY 25 IN SANTA FE, NEW MEXICO}

By E. L. Hogue

On July 25 a flow of moist, unstable air from the Gulf of Mexico caused heavy precipitation, which was centered 2 miles northeast of downtown Santa Fe in the foothills of the Sangre de Christo Mountains. Intense precipitation was orographically influenced as is often the case with severe storms in the Santa $\mathrm{Fe}$ area. Most precipitation occurred between 1 p.m. and 5 p.m. The storm total of precipitation recorded by the U.S. Weather Bureau gage at Los Cerros Reservoir in the eastern part of Santa Fe was 3.51 inches; maximum observed precipitation (in a storage-type rain gage) was 4.0 inches; maximum recorded intensity was 2.08 inches per hour. Figure 19 shows the pattern and distribution of rainfall for the storm and the flood-determination points. The isohyets are based on bucket-survey readings obtained by employees of the U.S. Geological Survey, the U.S. Soil Conservation Service, and the office of the State Engineer of New Mexico.

The Santa Fe River flows westerly through the city and the channel is normally dry except during periods of snowmelt or storm runoff. Between Twomile Reservoir and downtown Santa Fe there are 6 square miles of contributing, unregulated drainage. Most of the flood flow came from Canada Ancha, a tributary that enters the Santa Fe River from the north, 1.4 miles downstream from Twomile Reservoir. During the storm period there was no significant outflow from Twomile Reservoir.

Most of the flood damage was confined to a narrow strip along the Santa Fe River between the mouth of Canada Ancha and the mouth of Arroyo Mascaras. Constriction of the channel by bridges and culverts augmented by a heavy accumulation of debris and vegetation in the channel forced flood water into adjacent streets along the right bank. Flooding was most severe in the business area immediately north of the Jefferson Street crossing. Flood water reached a depth of 4 feet in some buildings. Arroyo Mascaras also flooded Jefferson Street at the bridge crossing north of the business district, halting traffic for several hours.

A tabulation of peak discharges measured by indirect methods is shown in table 15. Flood frequency relationships have not been determined for this urbanized area. Previous floods occurred in $1872,1904,1914,1921,1929$, and 1957. The flood of 1904, prior to the construction of Twomile Reservoir, destroyed three bridges in downtown Santa Fe.

A summary of flood damage compiled by the U.S. Army Corps of Engineers for the floods of July 25 is given below: 
Residential

Type of damage

Estimated damage

Business

$\$ 35,000$

134,000

Utilities

12,000

Highways, streets, and roads

74,000

Flood control and drainage structures

127,000

Miscellaneous

15,000

Total

$\$ 397,000$

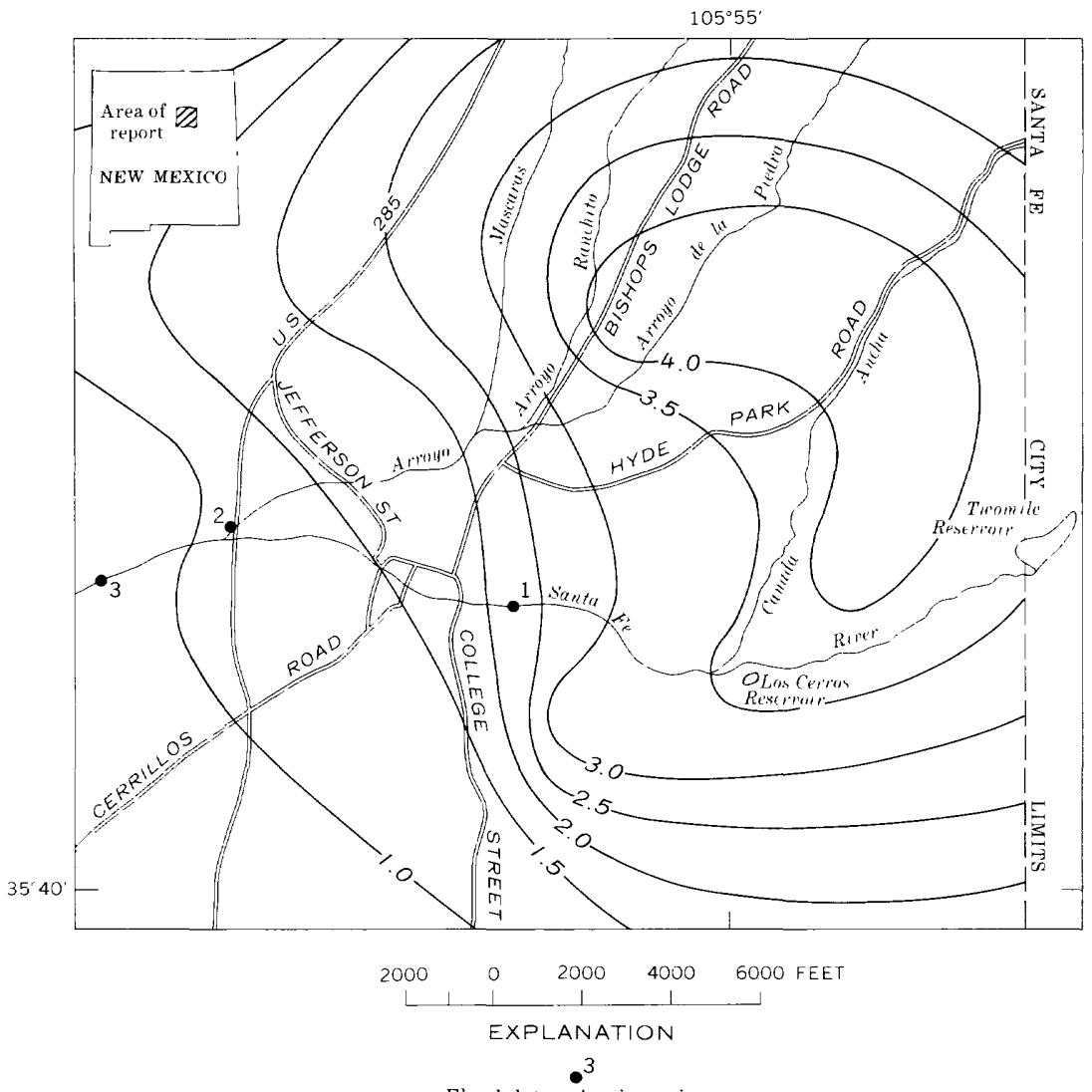

Flood-determination point

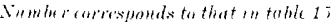

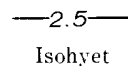

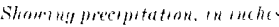

Figure 19.-Flood area; location of flood-determination points and isohyets for July 25 (from map furnished by the U.S. Army Corps of Engineers), floods of July 25 in Santa Fe, N. Mex. 
TABLE 15.-Flood stages and discharges, July 25 at Santa Fe, N. Mex.

\begin{tabular}{|c|c|c|c|c|c|c|c|}
\hline \multirow{3}{*}{ No. } & \multirow{3}{*}{$\begin{array}{l}\text { Stream and place } \\
\text { of determination }\end{array}$} & \multirow{3}{*}{$\begin{array}{l}\text { Drainage } \\
\text { area } \\
\text { (sq mi) }\end{array}$} & \multicolumn{5}{|c|}{ Maximum floods } \\
\hline & & & \multicolumn{2}{|c|}{$\begin{array}{c}\text { Known before } \\
\text { July } 1968\end{array}$} & \multirow{2}{*}{$\begin{array}{l}\text { July } \\
1968\end{array}$} & \multirow{2}{*}{$\begin{array}{c}\text { Gage } \\
\text { height } \\
\text { (feet) }\end{array}$} & \multirow{2}{*}{$\frac{\text { Discharge }}{\text { Cfs }}$} \\
\hline & & & Period & Year & & & \\
\hline 1 & $\begin{array}{l}\text { Santa Fe River at } \\
\text { Santa Fe, } 1,500 \mathrm{ft} \\
\text { upstream from } \\
\text { College Street. }\end{array}$ & ${ }^{1} 6.1$ & $1952-57$ & 1957 & 25 & . & $\begin{array}{l}1,450 \\
3,420\end{array}$ \\
\hline 2 & $\begin{array}{l}\text { Arroyo Mascaras at } \\
\text { Santa Fe, } 100 \mathrm{ft} \text { up- } \\
\text { stream from mouth. }\end{array}$ & 6.27 & 1957 & 1957 & 25 & (n.... & $\begin{array}{l}3,040 \\
2,490\end{array}$ \\
\hline 3 & $\begin{array}{l}\text { Santa Fe River below } \\
\text { Arroyo Mascaras at } \\
\text { Santa Fe, } 0.5 \mathrm{mi} \\
\text { downstream from } \\
\text { Arroyo Mascaras. }\end{array}$ & ${ }^{1} 13.9$ & - & $\ldots$ & 25 & $\ldots$ & 6,110 \\
\hline
\end{tabular}

${ }^{1}$ Contributing area.

FLOODS OF AUGUST 1-2 IN SOUTHEASTERN UTAH

By G. E. PYPER

Thunderstorms were frequent throughout Utah from July 21 to August 28, with most of the State receiving substantial amounts of precipitation. Fifteen precipitation stations in the State, two with records beginning in the 1890 's, reported the wettest August of record. Many isolated floods occurred around the State but caused little damage.

Only the record-breaking rainfall and floods that occurred on August 1-2 in southeastern Utah are considered in this report. Hardest hit was the San Juan River basin. The area affected is shown in figure 20.

The severity of the rainfall and of the floods was unusual for Utah, and hence they were of considerable hydrologic interest. The floods were caused by an intense thunderstorm following 11 days of antecedent rainfall that left the upper soil zone near saturation; therefore, most of the cloudburst rainfall on August 1 became surface runoff.

The severe thunderstorm of August 1 centered on the mountains north of Bluff and Blanding and west of Monticello. The precipitation recorded at Bluff for August 1 was 0.40 inch for 24 hours, whereas the Monticello gage recorded 3.38 inches and the Blanding gage recorded 4.48 inches. New precipitation-intensity records at Blanding were recorded on August 1 for the following periods :

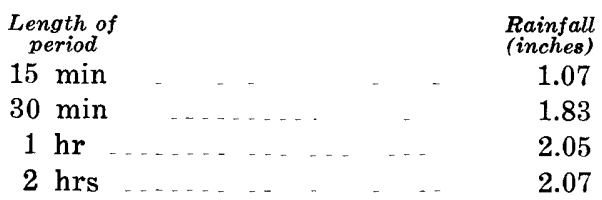

$\begin{gathered}\text { Beginning } \\ \text { of period }\end{gathered}$
$1: 45$ p.m.
$9: 42$ a.m.
$9: 42$ a.m.
$9: 42$ a.m.




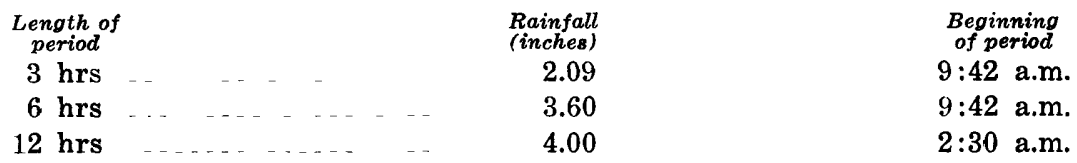

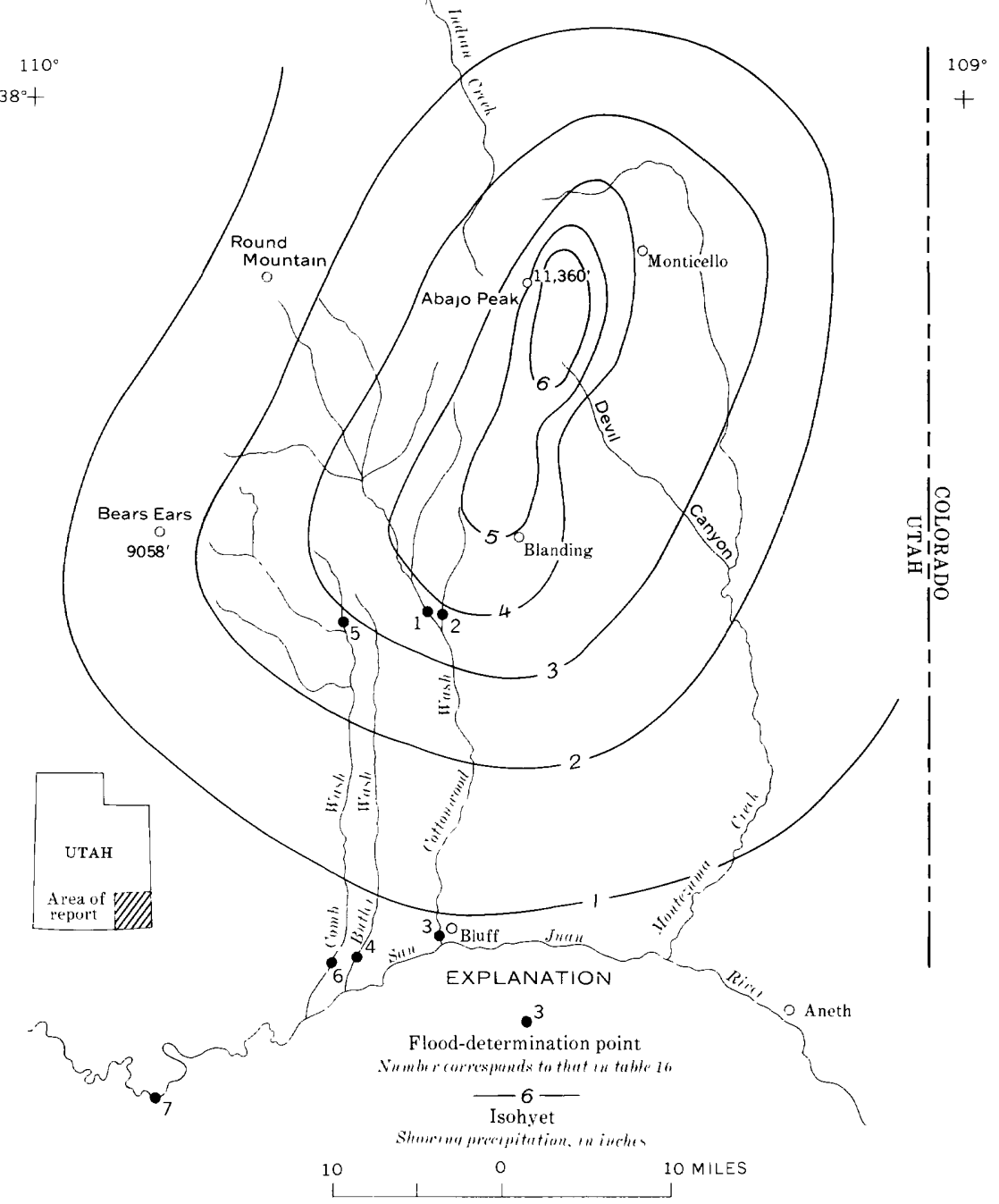

Figure 20.-Flood area; location of flood-determination points and isohyets for 24-hour precipitation, August 1-2, floods of August 1-2 in southeastern Utah.

A 24-hour catch of 4.59 inches at Blanding (recorded August 1-2), although not a record for the State, was more than 1.6 times as much as that of the U.S. Weather Bureau "100-year 24- 
hour" storm at Blanding, and it was near the normal May-September (5-month) catch of 5 inches. The precipitation for a 24hour maximum May-October storm at Blanding for return intervals of 50 and 100 years is 2.5 and 2.8 inches, respectively (U.S. Weather Bureau, 1961).

The peak discharge of $42,100 \mathrm{cfs}$ at the gaging station Cottonwood Wash at Bluff (No. 3, table 16), from a drainage area of 340 square miles, was more than 10 times the previous maximum in the 10 years of record. Twenty-one miles upstream, at Cottonwood Wash near Blanding (No. 1, table 16), the peak discharge of $20,500 \mathrm{cfs}$ from 205 square miles was more than twice the previous maximum in the 10 years of record.

The recurrence interval at most stations exceeded 50 years. A summary of the flood stage and discharge for the points in figure 20 is shown in downstream order in table 16 .

The area affected hy flooding is very sparsely populated, and no lives were lost. Damage was limited to roads, bridges, and rangelands, except in Bluff where businesses, homes, and farmlands were damaged. Damage in Bluff was estimated by the U.S. Soil Conservation Service at $\$ 33,700$.

Although the precipitation was intense also in the area east of Monticello and Blanding (Montezuma Creek drainage), the flooding was not severe. The peak discharge in Montezuma Creek had a recurrence interval of less than 10 years.

TABLE 16.-Flood stages and discharges, August 1-2, San Juan River basin, southeastern Utah

\begin{tabular}{|c|c|c|c|c|c|c|c|c|}
\hline \multirow{4}{*}{ No. } & \multirow{4}{*}{$\begin{array}{l}\text { Stream and place } \\
\text { of determination }\end{array}$} & \multirow{4}{*}{$\begin{array}{c}\text { Drainage } \\
\text { area } \\
\text { (sq mi) }\end{array}$} & \multicolumn{6}{|c|}{ Maximum floods } \\
\hline & & & \multirow{2}{*}{\multicolumn{2}{|c|}{$\begin{array}{l}\text { Known before } \\
\text { August } 1968\end{array}$}} & \multirow{3}{*}{ August } & \multirow{3}{*}{$\begin{array}{c}\text { Gage } \\
\text { height } \\
\text { (feet) }\end{array}$} & \multicolumn{2}{|c|}{ Discharge } \\
\hline & & & & & & & \multirow[b]{2}{*}{ Cfs } & \multirow{2}{*}{$\begin{array}{c}\text { Recur- } \\
\text { rence } \\
\text { interva } \\
\text { (years) }\end{array}$} \\
\hline & & & Period & Year & & & & \\
\hline 1 & $\begin{array}{l}\text { Cottonwood Wash } \\
\text { near Blanding. }\end{array}$ & 205 & $1959-68$ & 1963 & $i$ & $\begin{array}{r}118.55 \\
20.68\end{array}$ & $\begin{array}{r}8,650 \\
20,500\end{array}$ & 26.4 \\
\hline 2 & $\begin{array}{l}\text { Unnamed tributa- } \\
\text { ry to Cotton- } \\
\text { wood Wash } \\
\text { near Blanding. }\end{array}$ & 13.6 & ....... & ... & 1 & $\ldots$ & 7,300 & $\left(^{3}\right)$ \\
\hline 3 & $\begin{array}{l}\text { Cottonwood Wash } \\
\text { at Bluff. }\end{array}$ & 340 & $\begin{array}{c}1959-68 \\
-\end{array}$ & 1963 & $i$ & $\begin{array}{r}+15.24 \\
18.38\end{array}$ & $\begin{array}{r}4,060 \\
42,100\end{array}$ & 211.8 \\
\hline 4 & $\begin{array}{l}\text { Butler Wash near } \\
\text { Bluff. }\end{array}$ & 54 & $\begin{array}{c}1959-68 \\
-\end{array}$ & 1963 & 1 & $\begin{array}{l}16.10 \\
14.87\end{array}$ & $\begin{array}{r}1,000 \\
820\end{array}$ & $\left({ }^{3}\right)$ \\
\hline 5 & $\begin{array}{l}\text { Comb Wash near } \\
\text { Blanding. }\end{array}$ & 10.3 & $\begin{array}{r}1959-68 \\
-\end{array}$ & 1964 & 1 & $\begin{array}{l}12.80 \\
14.48\end{array}$ & $\begin{array}{l}1,400 \\
3,430\end{array}$ & $\left({ }^{3}\right)$ \\
\hline 6 & $\begin{array}{l}\text { Comb Wash near } \\
\text { Bluff. }\end{array}$ & 280 & $\begin{array}{c}1959-68 \\
-9\end{array}$ & 1959 & 1 & $\begin{array}{l}3.32 \\
5.57\end{array}$ & $\begin{array}{l}2,840 \\
8,390\end{array}$ & 23.1 \\
\hline \multirow[t]{2}{*}{7} & $\begin{array}{l}\text { San Juan River } \\
\text { near Bluff. }\end{array}$ & 23,000 & $\begin{array}{l}1914-17 \\
1927-68\end{array}$ & 1927 & & 32.0 & 70,000 & $\ldots$ \\
\hline & & & & $\ldots$ & 2 & 14.06 & 17,500 & 2 \\
\hline
\end{tabular}

1 At site $300 \mathrm{ft}$ upstream at different datum.

2 Ratio of peak discharge to 50 -year flood.

${ }^{3}$ Undefined by flood-frequency data but probably greater than 50-year flood.

4 At site of old highway crossing at different datum. 


\section{FLOODS OF AUGUST 3 AND 4 IN GLOBE, ARIZONA}

\section{By S. C. Brown}

On August 3 a storm cell centered over Globe, and 2.56 inches of rain fell at the U.S. Weather Bureau precipitation station in the downtown area (fig. 21) between 2:45 p.m. and 4:10 p.m. A resident about 0.6 mile northeast of the Weather Bureau gage reported 3.3 inches of rain in 45 minutes. Pinal Creek, choked with debris that had washed in from its tributary canyons, overflowed its banks and flooded a few stores and part of U.S. Highway 60 and 70 in downtown Globe; flooding in the northerly draining tributary canyons was severe.

A child was drowned in the rapidly rising water when he and his sister attempted to cross a street about a quarter of a mile below the confluence of Graveyard and Echo Canyons (No.

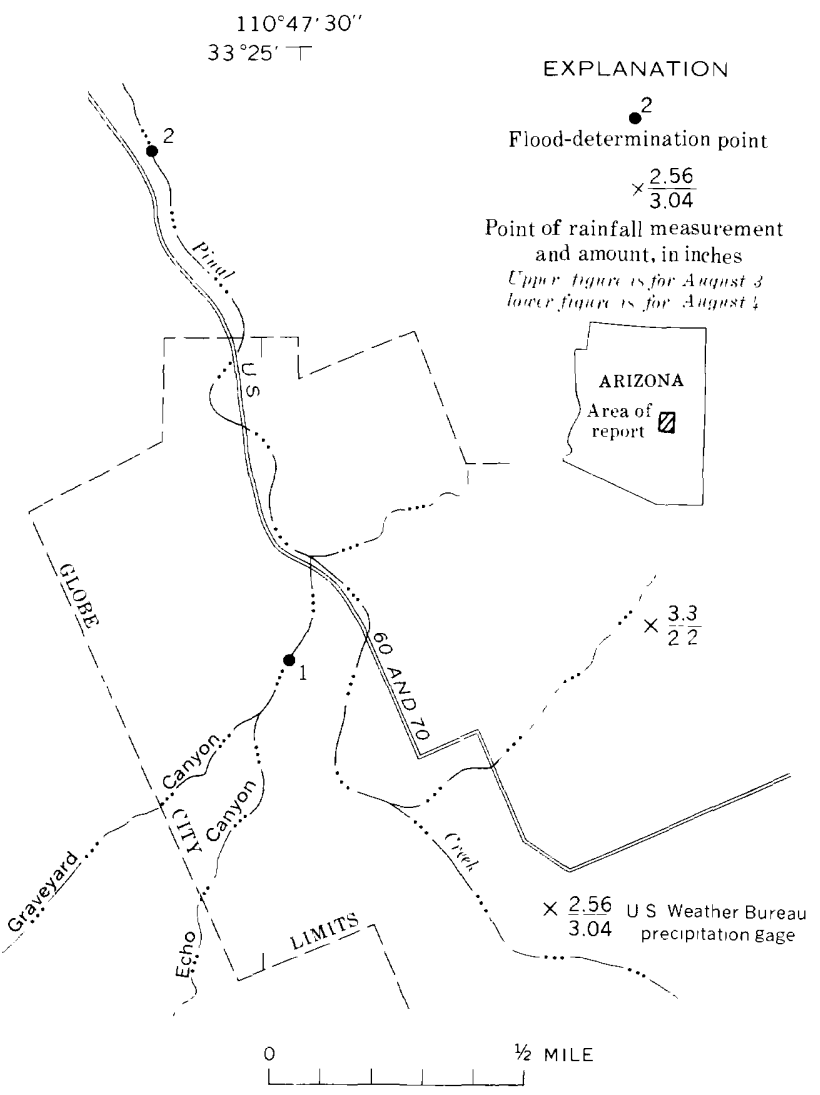

FigURE 21.-Flood area; location of flood-determination points and rainfalldata sites, floods of August 3 and 4 in Globe, Ariz. 
1, fig. 22) ; the peak discharge estimated from channel properties, was about $3,000 \mathrm{cfs}$ from a drainage area of 1.2 square miles. A resident who had lived in Graveyard Canyon since 1933 described the flood as the greatest of any he had seen in the canyon. According to local reports, 16 automobiles were swept down Pinal Creek, bridge and culvert openings were choked with debris and some were overtopped, roofs were ripped from buildings by the high winds that preceded the rain, and electrical power was off for several hours on August 3 and 4. The Globe water system sustained about $\$ 15,000$ in damage, and a reported $\$ 25,000$ in damage occurred at a local automobile agency.

An indirect measurement of the peak discharge of Pinal Creek about half a mile north of the Globe city limits (No. 2, fig. 22) was calculated as 7,400 cfs from a drainage area of 34.4 square miles; the discharge has a recurrence interval of about 25 years. On July 29, 1954, a peak discharge of 8,130 cfs was measured at about the same site and on August 17, 1904, a peak discharge of $13,200 \mathrm{cfs}$ was measured at a site nearby, where the drainage area was 33.4 square miles.

Another heavy rainstorm on August 4 began about 7:00 p.m. and 3.04 inches of rain was recorded at the U.S. Weather Bureau precipitation station. According to local observers, 2 inches of rain fell in the first hour. The rain gage 0.6 mile northeast of the Weather Bureau gage recorded 2.2 inches during the storm. Although Pinal Creek continued to flow on August 4, it did not overflow its banks.

\section{FLOODS OF AUGUST 5-6 IN CEDAR RIVER BASIN, IOWA}

By HaRlan H. Schwob

Heavy rainfall on the night of August 4-5 caused a flash flood in the Dry Run Creek basin (drainage area of $24.2 \mathrm{sq} \mathrm{mi}$ ) in Cedar Falls, Iowa. The most rainfall reported was 7.44 inches at Parkersburg about 17 miles west of Cedar Falls. However, much heavier rainfall in a few hours was probable over the creek basin if unofficial estimates are considered. The severity of the resulting flood also tends to confirm this probability. Figure 22 shows the location of the creek, the gaging stations, and the rainfall amounts reported by the U.S. Weather Bureau.

Two indirect measurements of discharge were made in the Dry Run Creek basin. Both peak discharges were much greater than the estimate of the 50-year flood (table 17). Floods at regular gaging stations in the vicinity (Nos. 1, 4, 5, table 17) were comparatively small. 


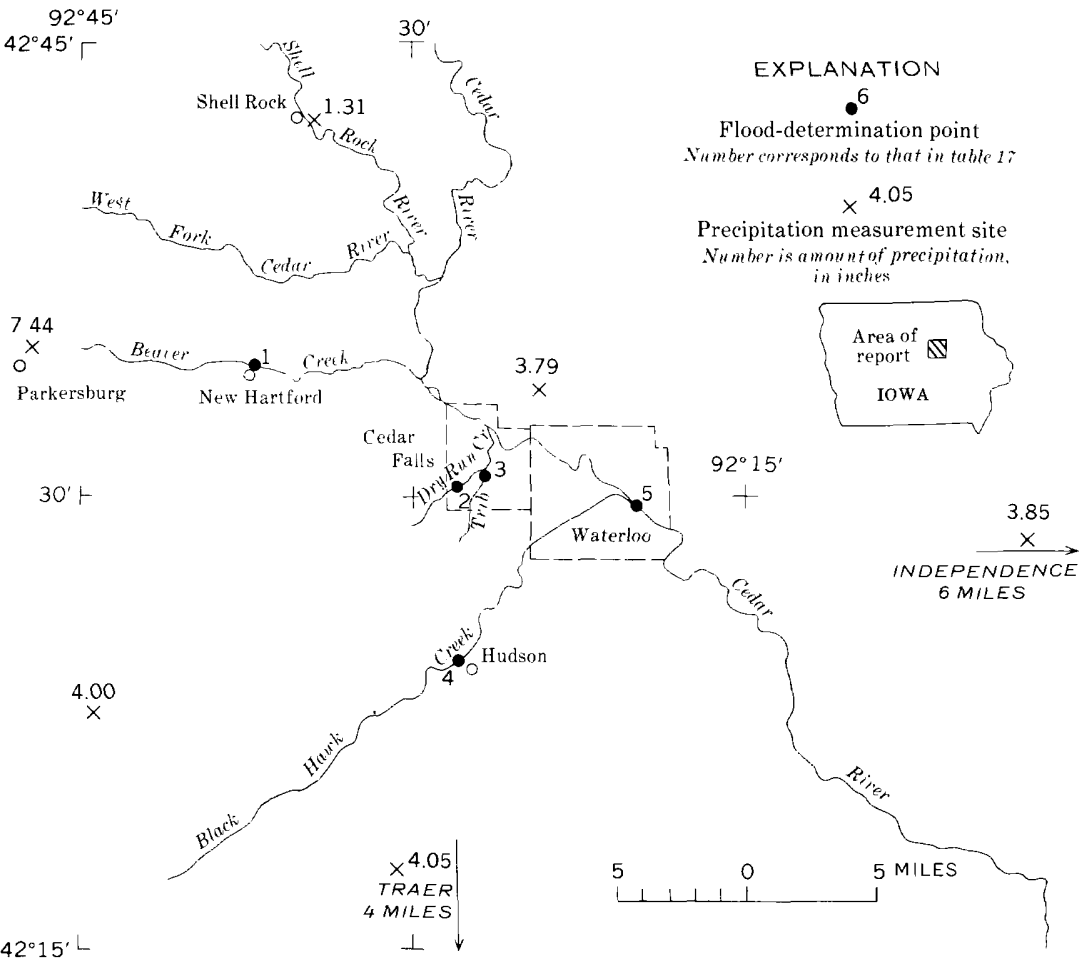

FIGURE 22.-Flood area; location of flood-determination points, floods of August 5-6 in Cedar River basin, Iowa.

TABLE 17.-Flood stages and discharges, August 5-6 in Cedar River basin, lowa

\begin{tabular}{|c|c|c|c|c|c|c|c|c|}
\hline \multirow[b]{4}{*}{ No. } & \multirow{4}{*}{$\begin{array}{l}\text { Stream and place } \\
\text { of determination }\end{array}$} & \multirow{4}{*}{$\begin{array}{l}\text { Drainage } \\
\text { area } \\
\text { (sq mi) }\end{array}$} & \multicolumn{6}{|c|}{ Maximum floods } \\
\hline & & & \multirow{2}{*}{\multicolumn{2}{|c|}{$\begin{array}{l}\text { Known before } \\
\text { August } 1968\end{array}$}} & \multirow{3}{*}{$\underset{1968}{\text { August }}$} & \multirow{3}{*}{$\begin{array}{c}\text { Gage } \\
\text { height } \\
\text { (feet) }\end{array}$} & \multicolumn{2}{|c|}{ Discharge } \\
\hline & & & & & & & \multirow[b]{2}{*}{ Cfs } & \multirow{2}{*}{$\begin{array}{l}\text { Recur- } \\
\text { rence } \\
\text { interval } \\
\text { (years) }\end{array}$} \\
\hline & & & Period & Year & & & & \\
\hline 1 & $\begin{array}{l}\text { Beaver Creek at } \\
\text { New Hartford. }\end{array}$ & 347 & $1946-68$ & 1947 & 6 & $\begin{array}{l}13.5 \\
9.39\end{array}$ & $\begin{array}{r}18,000 \\
2,510\end{array}$ & $-<$ \\
\hline 2 & $\begin{array}{l}\text { Dry Run Creek } \\
\text { at Cedar Falls. }\end{array}$ & 8.46 & $\ldots \ldots$ & $\cdots$ & 5 & 2892.5 & 7,600 & ${ }^{1} 6.3$ \\
\hline 3 & $\begin{array}{c}\text { Dry Run Creek } \\
\text { tributary at } \\
\text { Cedar Falls. }\end{array}$ & 9.71 & $\ldots \ldots$ & 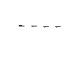 & 5 & 2870.71 & 5,170 & ${ }^{1} 4.1$ \\
\hline 4 & $\begin{array}{c}\text { Black Hawk } \\
\text { Creek at } \\
\text { Hudson. }\end{array}$ & 303 & $1953-68$ & 1960 & 6 & $\begin{array}{l}16.93 \\
15.26\end{array}$ & $\begin{array}{l}9,000 \\
3,470\end{array}$ & $<2$ \\
\hline \multirow[t]{2}{*}{5} & Cedar River at & 5,146 & 1929,33 & 1961 & $\cdots$ & 21.86 & 76,700 & $\cdots$ \\
\hline & & & $\begin{array}{r}1941-68 \\
\ldots \ldots\end{array}$ & - - - & 5 & 9.64 & 13,800 & $<2$ \\
\hline
\end{tabular}

${ }^{1}$ Ratio to 50-year flood.

2 Elevation in feet above mean sea level (datum of 1929) at downstream side at bridge. 
The flood elevations through Cedar Falls were determined after the flood peak. These data are shown in Table 18 and can be used to plot a flood profile along the reach of the creeks within the city.

One life was lost and considerable damage resulted from the floods. Several homes and small businesses were flooded, highways and streets were temporarily closed, and equipment from the city storage yard was washed into the creek. At one point on the tributary to Dry Run Creek a manhole on a sanitary sewer located under the creek bed was broken and raw sewage entered the creek until repairs were made.

TABLE 18.-A ugust flood elevations, in feet above mean sea level, in Dry Run Creek basin, Cedar Falls, Iowa

\begin{tabular}{|c|c|c|c|}
\hline \multirow[b]{2}{*}{ Mile } & \multirow[b]{2}{*}{ Place of determination } & \multicolumn{2}{|c|}{ Elevation } \\
\hline & & Downstream & Upstream \\
\hline \multicolumn{4}{|c|}{ Dry Run Creek } \\
\hline 0 & $\begin{array}{l}\text { Mouth of Dry Run Creek at Cedar River, } \\
\text { mile } 194.9\end{array}$ & 852.4 & \\
\hline .05 & C.R.I. \& P. RR. bridge & 852.8 & 853.0 \\
\hline .42 & Waterloo Road bridge & 859.8 & 860.3 \\
\hline .53 & City storage yard $\ldots$ & 861.1 & $\ldots$ \\
\hline .60 & At end of 17 th St. & 862.8 & \\
\hline .72 & Upstream of Rainbow Dr. & & 864.4 \\
\hline .83 & Intersection 20th St. \& Bluff St. & 865.8 & \\
\hline 1.15 & Downstream of Seerley Blvd. ........ & 868.4 & \\
\hline 1.19 & Upstream of Seerley Blvd. & & 870.4 \\
\hline 1.27 & Main Street bridge & 870.8 & 873.6 \\
\hline 1.56 & 27th Street bridge (Highway 58) & 876.5 & 877.9 \\
\hline 2.40 & At Panther Lane - & 889.7 & \\
\hline 2.63 & Highway 58 bridge (Hudson Rd.) & 892.5 & 895.4 \\
\hline \multicolumn{4}{|c|}{ Dry Run Creek tributary } \\
\hline 1.22 & Tributary mouth & 870.6 & \\
\hline 1.40 & Highway 218 bridge & 870.7 & 872.9 \\
\hline 1.86 & Main Street bridge & 879.1 & \\
\hline 1.90 & C.G.W. RR. bridge & $\ldots$ & 881.4 \\
\hline
\end{tabular}

\section{FLOODS OF AUGUST I5 IN WESTERN NEBRASKA}

By H. D. Brice

As much as 6.40 inches of rain fell in the southeastern part of the Nebraska panhandle within a 12-hour period on August 15, according to U.S. Weather Bureau measurements (fig. 23). Even larger amounts-as much as 9 inches-were reported unofficially. Because the recurrence interval of a point rainfall of 4.5 inches in 12 hours in this part of Nebraska is 100 years (U.S. Weather Bureau, 1961), the recurrence interval of amounts as large as those recorded and reported would be much greater than 100 years. 


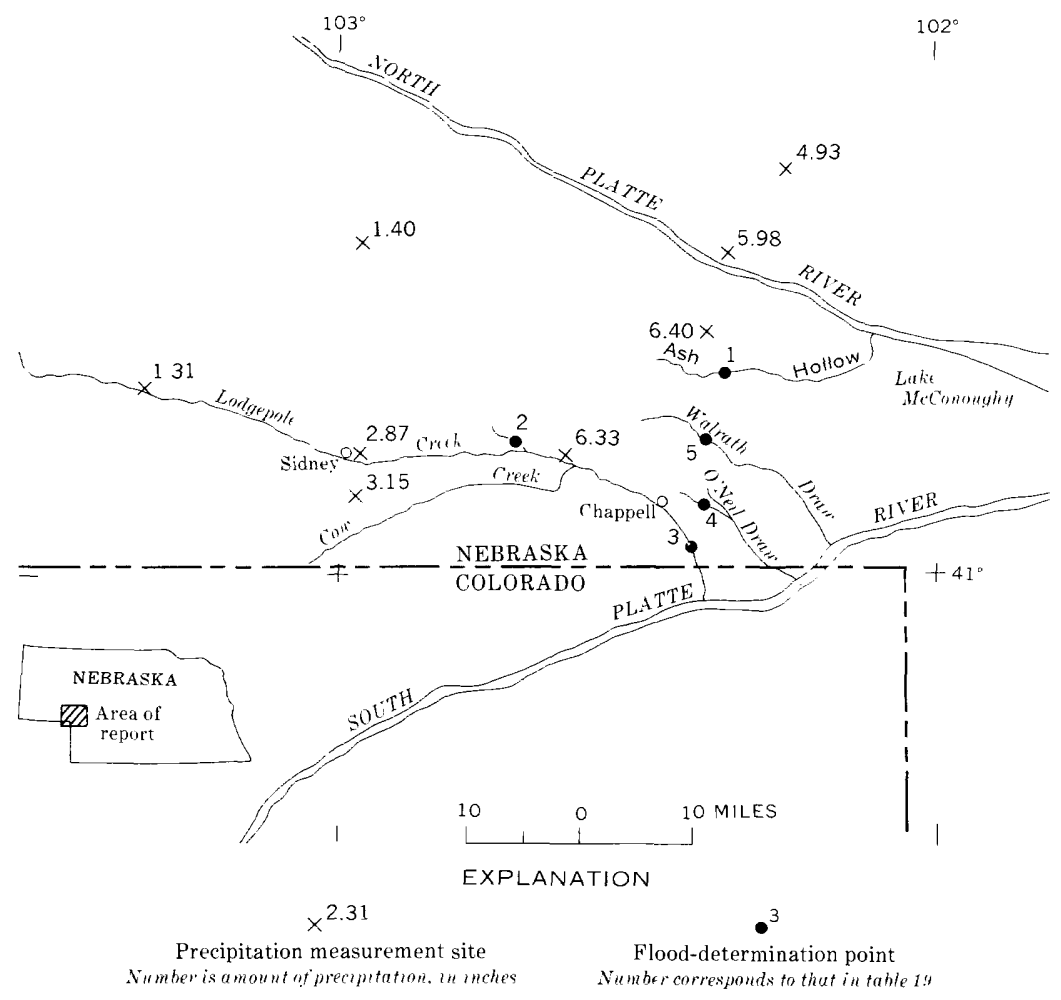

FIGURE 23.-Flood area; location of flood-determination points and rainfall data sites, floods of August 15 in western Nebraska.

Peak discharges were measured at five sites in the storm area (table 19). The peak on Lodgepole Creek at Ralton (No. 3, table 19) was about four times the previous maximum and had an estimated recurrence interval greater than 100 years. As flood-frequency relations have not been defined for streams in this area and no records of earlier peak discharges are available, recurrence intervals could not be estimated for the other four sites.

Reports of damages were most numerous from the vicinity of Chappell. U.S. Highway 30 near that town was under water and several sections of roadbed were eroded. South of Chappell, a gully 100 feet wide and 6 feet deep was incised across a 90-acre field. Also, many bridges and culverts in the area were washed out. Although a small dam west of Chappell gave way, four ranch dams north of town held and were credited with preventing serious flooding in the town. A few elderly area residents, whose memories spanned about 75 years, stated that they had 
never experienced such a severe storm nor seen such great flood damage before.

TABLE 19.-Flood stages and discharges, August 15 in western Nebraska

\begin{tabular}{|c|c|c|c|c|c|c|c|c|}
\hline \multirow[b]{4}{*}{ No. } & \multirow{4}{*}{$\begin{array}{l}\text { Stream and place } \\
\text { of determination }\end{array}$} & \multirow{4}{*}{$\begin{array}{l}\text { Drainage } \\
\text { area } \\
(\mathrm{sq} \mathrm{mi})\end{array}$} & \multicolumn{6}{|c|}{ Maximum floods } \\
\hline & & & \multirow{2}{*}{\multicolumn{2}{|c|}{$\begin{array}{l}\text { Known before } \\
\text { August } 1968\end{array}$}} & \multirow{3}{*}{$\underset{1968}{\text { August }}$} & \multirow{3}{*}{$\begin{array}{l}\text { Gage } \\
\text { height } \\
\text { (feet) }\end{array}$} & \multicolumn{2}{|c|}{ Discharge } \\
\hline & & & & & & & & Recur- \\
\hline & & & Period & Year & & & Cfs & $\begin{array}{c}\text { interval } \\
\text { (years) }\end{array}$ \\
\hline 1 & $\begin{array}{l}\text { Ash Hollow near } \\
\text { Oshkosh. }\end{array}$ & 48.4 & - - - . . . - & $\cdots$ & 15 & $\ldots$ & 3,440 & $\cdots$ \\
\hline 2 & $\begin{array}{l}\text { Tributary to } \\
\text { Lodgepole Creek } \\
\text { near Lodgepole. }\end{array}$ & 15.8 & $\ldots \ldots$ & - . - & 15 & - & 820 & - \\
\hline 3 & $\begin{array}{l}\text { Lodgepole Creek } \\
\text { at Ralton. }\end{array}$ & 3,307 & $1931,1951-68$ & 1951 & 15 & $\begin{array}{c}5.70 \\
6.49\end{array}$ & $\begin{array}{l}1,150 \\
4,560\end{array}$ & 100 \\
\hline 4 & $\begin{array}{l}\text { Tributary to } \\
\text { O'Neil Draw } \\
\text { near Chappell. }\end{array}$ & 2.07 & $\ldots$ & & 15 & - & 302 & - \\
\hline 5 & $\begin{array}{l}\text { Walrath Draw } \\
\text { near Chappell. }\end{array}$ & 69.5 & $-\ldots \ldots$. & & 15 & & 2,200 & - \\
\hline
\end{tabular}

\section{FLOODS OF AUGUST 28-29 IN THE TEXAS HIGH PLAINS}

By L. G. Stearns

Heavy rains of up to 8 inches fell on Prairie Dog Town Fork Red River basin (fig. 24) between Canyon and Estelline during the afternoon and night of August 28. On August 28, as a result of these heavy rains, a $\$ 2$ million train wreck 15 miles northwest

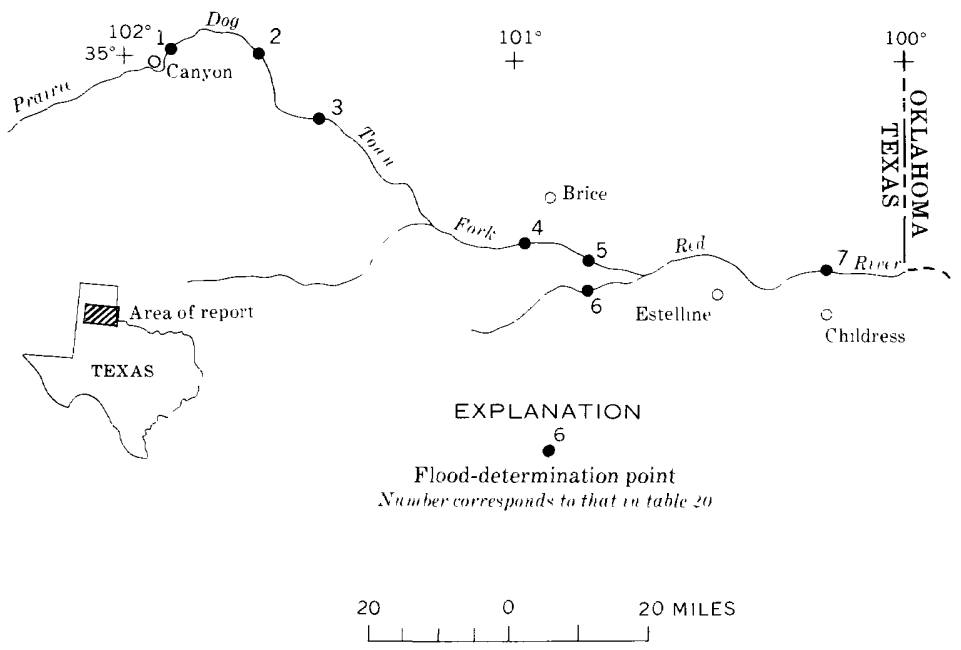

Figure 24.-Flood area; location of flood-determination points, floods of August 28-29 in the Texas High Plains. 
of Childress claimed one life. The derailment occurred at a point where the railroad crosses a small draw.

The U.S. Weather Bureau reported 7.87 inches of rain at Canyon, and all highways through Canyon were closed for a time during and immediately after the storm. A peak stage of 20.54 feet (determined from drift marks) occurred at the discontinued stream-gaging station Prairie Dog Town Fork Red River near Canyon (No. 1, table 20). Above Palo Duro State Park (No. 2, table 20) survey levels to drift at the slope-area site show that the crest of the flood was about 1.5 feet lower than the flood of June 1960.

The maximum discharges at gaging stations 2 and 3 in table 20 were the most outstanding observed in the basin during this period of flooding.

TABLE 20.-Flood stages and discharges, August 28-29 in the Red River basin, Texas High Plains

\begin{tabular}{|c|c|c|c|c|c|c|c|c|}
\hline \multirow[b]{4}{*}{ No. } & \multirow{4}{*}{$\begin{array}{l}\text { Stream and place } \\
\text { of determination }\end{array}$} & \multirow{4}{*}{$\begin{array}{l}\text { Drainage } \\
\text { area } \\
\text { (sq mi) }\end{array}$} & \multicolumn{6}{|c|}{ Maximum floods } \\
\hline & & & \multirow{2}{*}{\multicolumn{2}{|c|}{$\begin{array}{l}\text { Known before } \\
\text { August } 1968\end{array}$}} & \multirow{3}{*}{ August } & \multirow{3}{*}{$\begin{array}{c}\text { Gage } \\
\text { height } \\
\text { (feet) }\end{array}$} & \multicolumn{2}{|c|}{ Discharge } \\
\hline & & & & & & & & Recur- \\
\hline & & & Period & Year & & & Cfs & $\begin{array}{c}\text { interval } \\
\text { (years) }\end{array}$ \\
\hline 1 & $\begin{array}{l}\text { Prairie Dog Town Fork } \\
\text { Red River near } \\
\text { Canyon. }\end{array}$ & $\begin{array}{l}3,369 \\
1711\end{array}$ & $\begin{array}{l}1905-68 \\
\cdots\end{array}$ & $\begin{array}{l}1951 \\
\ldots-\end{array}$ & 28 & $\begin{array}{l}20.31 \\
20.54\end{array}$ & $\begin{array}{l}15,200 \\
15,500\end{array}$ & 14 \\
\hline 2 & $\begin{array}{l}\text { Prairie Dog Town Fork } \\
\text { Red River above Palo } \\
\text { Duro State Park } \\
\text { near Canyon. }\end{array}$ & ${ }^{1} 743$ & $1951-68$ & 1960 & 28 & 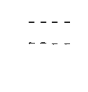 & $\begin{array}{l}52,700 \\
48,000\end{array}$ & 21.6 \\
\hline 3 & $\begin{array}{l}\text { Prairie Dog Town Fork } \\
\text { Red River near } \\
\text { Wayside. }\end{array}$ & $\begin{array}{l}4,211 \\
1930\end{array}$ & $\ldots$ & - - & 28 & 13.0 & 58,000 & 21.7 \\
\hline 4 & $\begin{array}{l}\text { Prairie Dog Town Fork } \\
\text { Red River near } \\
\text { Brice. }\end{array}$ & $\begin{array}{r}6,082 \\
11,581\end{array}$ & $\begin{array}{l}1906-68 \\
1958-68\end{array}$ & $\begin{array}{l}1957 \\
1960\end{array}$ & 28 & $\begin{array}{l}19.2 \\
12.20 \\
12.6\end{array}$ & $\begin{array}{r}\left({ }^{3}\right) \\
49,000 \\
54,000\end{array}$ & 14 \\
\hline 5 & $\begin{array}{l}\text { Prairie Dog Town Fork } \\
\text { Red River near } \\
\text { Lakeview. }\end{array}$ & $\begin{array}{r}6,792 \\
12,023\end{array}$ & $\begin{array}{c}1963-68 \\
-\quad \ldots\end{array}$ & 1965 & $\overline{29}$ & $\begin{array}{r}10.50 \\
9.10\end{array}$ & $\begin{array}{l}37,900 \\
\mathbf{5 1 , 0 0 0}\end{array}$ & $\left(\mathbf{l}^{3}\right)$ \\
\hline 6 & $\begin{array}{l}\text { Little Red River near } \\
\text { Turkey. }\end{array}$ & 139 & $\cdots-$ & $\ldots$ & 28 & 13.48 & 3,570 & 3 \\
\hline 7 & $\begin{array}{l}\text { Prairie Dog Town Fork } \\
\text { Red River near } \\
\text { Childress. }\end{array}$ & $\begin{array}{r}7,725 \\
12,956\end{array}$ & $1899-1968$ & 1957 & 29 & $\begin{array}{l}16.9 \\
11.15\end{array}$ & $38,{ }^{(3)}$ & (3) \\
\hline
\end{tabular}

1 Contributing area.

2 Ratio of peak discharge to 50-year flood.

3 Not determined.

\section{FLOODS OF AUGUST 28-31 IN THE VICINITY OF JACKSONVILLE, FLORIDA}

By JAMES W. RABON

A low-pressure disturbance which formed over central Florida on August 27 drifted aimlessly for several days before moving northward into Georgia at the end of the month. Resulting rains were especially heavy in Duval and Clay Counties (fig. 25), where 
considerable flooding occurred on small streams having drainage areas of less than 200 square miles.

The U.S. Weather Bureau at Imeson Airport, Jacksonville (fig. 25), recorded 15.17 inches of rainfall during the 4-day storm period of August 28-31, 7.82 inches of which fell on the 28th, 2.73 inches on the 29th, 3.88 inches on the 30 th, and 0.74 inches on the 31st. The recurrence interval of the 4-day storm rainfall at this point is about 100 years. Major damage resulted in the upper Cedar Creek basin where many houses and commercial establishments were flooded or were surrounded by flood waters, and 11 bridges were inundated to depths from a few inches to 4.5 feet (U.S. Army Corps of Engineers, 1969).

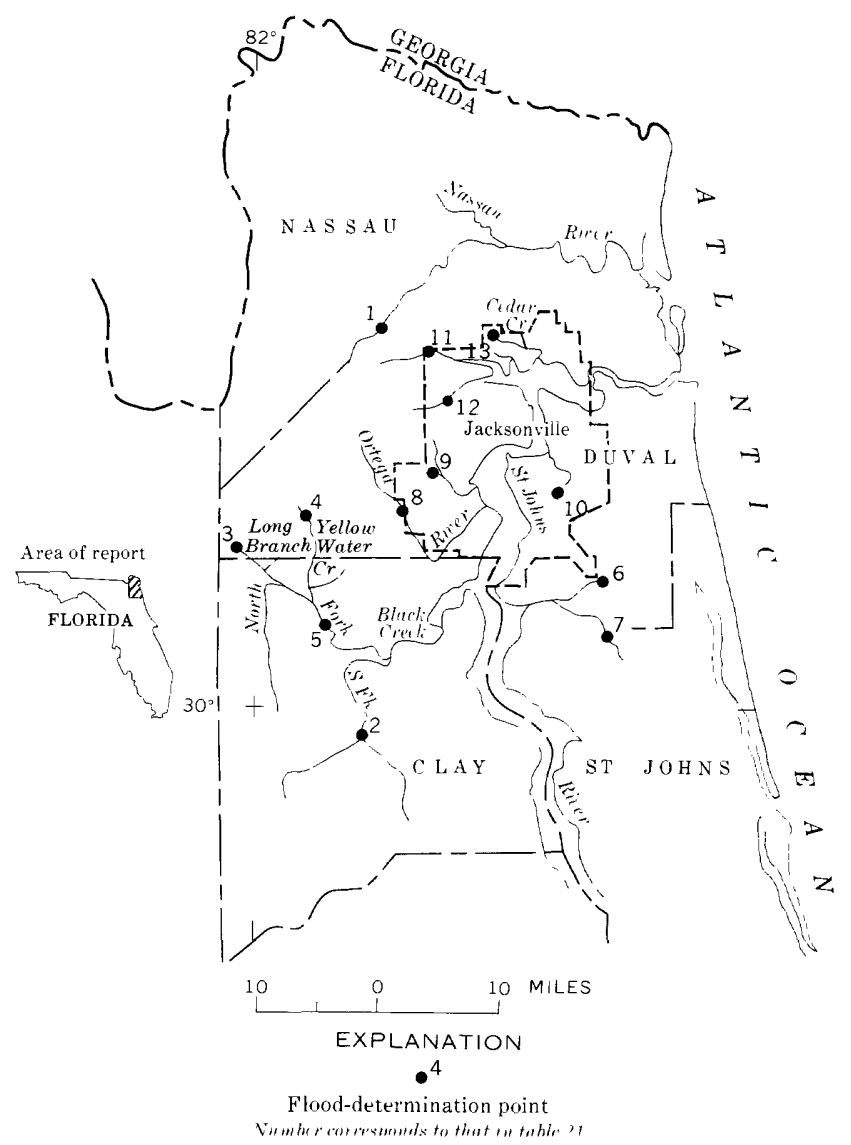

FIgURE 25.-Flood area; location of flood-determination points, floods of August $28-31$ in the vicinity of Jacksonville, Fla. 
Long-term flood record on streams in the Jacksonville urban area and Duval County are scarce. (See table 21.) The U.S. Geological Survey obtained highwater marks after two notable floods in May 1964 and in September 1964 (Hurricane Dora) and installed some crest-stage gages. The U.S. Army Corps of Engineers made additional field investigations, searched newspaper records, and interviewed local residents to supplement the data. Flood records for two long-term stations on the North Fork (No. 5, table 21) and South Fork (No. 2, table 21) of Black Creek, including data for the historic floods of 1919 and 1944, were used as references in determining relative frequencies for the August

TABLE 21.-Flood stages and discharges, August 28-31 in vicinity of Jacksonville, Fla.

\begin{tabular}{|c|c|c|c|c|c|c|c|c|}
\hline \multirow[b]{4}{*}{ No. } & \multirow{4}{*}{$\begin{array}{l}\text { Stream and place } \\
\text { of determination }\end{array}$} & \multirow{4}{*}{$\begin{array}{l}\text { Drainage } \\
\text { area } \\
(\mathrm{sq} \mathrm{mi})\end{array}$} & \multicolumn{6}{|c|}{ Flood peaks } \\
\hline & & & \multirow{2}{*}{\multicolumn{2}{|c|}{$\begin{array}{c}\text { Known before } \\
\text { August } 1968\end{array}$}} & \multirow{3}{*}{-August } & \multirow{3}{*}{$\begin{array}{c}\text { Elevation } \\
\text { (ft above } \\
\text { mean } \\
\text { sea level) }\end{array}$} & \multirow[b]{3}{*}{ Cfs } & \multirow{3}{*}{$\begin{array}{c}\text { Charge } \\
\begin{array}{c}\text { Recur- } \\
\text { rence } \\
\text { interval } \\
\text { (years) }\end{array}\end{array}$} \\
\hline & & & & & & & & \\
\hline & & & Period & Year & & & & \\
\hline \multicolumn{9}{|c|}{ Coastal basins between St. Marys River and St. Johns River. } \\
\hline 1 & $\begin{array}{l}\text { Thomas Creek near } \\
\text { Crawford. }\end{array}$ & 29.8 & $\begin{array}{l}1950 \\
1965-68 \\
\end{array}$ & $\begin{array}{l}1950 \\
1966 \\
-\end{array}$ & $\overline{3 i}$ & $\begin{array}{l}23.3 \\
18.97 \\
19.49\end{array}$ & $\begin{array}{r}(\mathbf{1}) \\
1,610 \\
1,920 \\
\end{array}$ & (1) \\
\hline \multicolumn{9}{|c|}{ St. Johns River basin } \\
\hline 2 & $\begin{array}{l}\text { South Fork Black } \\
\text { Creek near Penney } \\
\text { Farms. }\end{array}$ & 134 & $1940-68$ & $\begin{array}{r}1944 \\
21964 \\
31964\end{array}$ & $\begin{array}{l}-- \\
-- \\
\overline{3} \overline{1}\end{array}$ & $\begin{array}{l}36.15 \\
26.30 \\
29.86 \\
30.44\end{array}$ & $\begin{array}{r}13,900 \\
3,090 \\
\mathbf{6 , 4 4 0} \\
\mathbf{7 , 0 8 0}\end{array}$ & $\begin{array}{l}\cdots \\
\cdots \\
\cdots\end{array}$ \\
\hline 3 & $\begin{array}{l}\text { Long Branch at } \\
\text { Maxville. }\end{array}$ & 2.98 & $1965-66$ & 1965 & $\overline{30}$ & $\begin{array}{l}86.93 \\
87.90\end{array}$ & $\begin{array}{l}294 \\
380\end{array}$ & $\begin{array}{l}22 \\
22\end{array}$ \\
\hline 4 & $\begin{array}{l}\text { Yellow Water Creek } \\
\text { near Maxville. }\end{array}$ & 25.7 & $1958-68$ & $\begin{array}{rl}2 & 1964 \\
3 & 1964 \\
-\end{array}$ & $\begin{array}{l}-- \\
29\end{array}$ & $\begin{array}{l}59.81 \\
58.97 \\
59.27\end{array}$ & $\begin{array}{l}3,220 \\
1,890 \\
2,520\end{array}$ & 24 \\
\hline 5 & $\begin{array}{l}\text { North Fork Black } \\
\text { Creek near Middle- } \\
\text { burg. }\end{array}$ & 174 & $\begin{array}{l}1919-\overline{-}-\overline{1932-68}\end{array}$ & $\begin{array}{r}1919 \\
21964 \\
31964 \\
\ldots \ldots\end{array}$ & 30 & $\begin{array}{l}25.9 \\
24.53 \\
23.54 \\
23.94\end{array}$ & $\begin{array}{l}15,000 \\
12,000 \\
11,200 \\
11,800\end{array}$ & $\begin{array}{l} \\
\cdots \\
-14\end{array}$ \\
\hline 6 & $\begin{array}{l}\text { Big Davis Creek at } \\
\text { Bayard. }\end{array}$ & 13.6 & $1964-68$ & ${ }^{3} 1964$ & $\overline{29}$ & $\begin{array}{l}10.05 \\
10.47\end{array}$ & $\begin{array}{r}780 \\
1,170\end{array}$ & ii \\
\hline 7 & $\begin{array}{l}\text { Durbin Creek near } \\
\text { Durbin. }\end{array}$ & 36.7 & $1961-68$ & $\begin{array}{l}21964 \\
\quad 31964\end{array}$ & $3 \overline{0}$ & $\begin{array}{r}6.78 \\
10.10 \\
10.07\end{array}$ & $\begin{array}{l}1,350 \\
4,140 \\
4,050\end{array}$ & 50 \\
\hline 8 & $\begin{array}{l}\text { Ortega River near } \\
\text { Jacksonville. }\end{array}$ & 27.8 & $1964-68$ & 21964 & 30 & $\begin{array}{l}38.28 \\
39.32\end{array}$ & $\begin{array}{l}1,670 \\
2,950\end{array}$ & 35 \\
\hline 9 & $\begin{array}{l}\text { Wills Branch near } \\
\text { Marietta. }\end{array}$ & 7.04 & $1964-66$ & ${ }^{3} 1964$ & 30 & $\begin{array}{l}10.38 \\
10.55\end{array}$ & $\begin{array}{l}791 \\
820\end{array}$ & 23 \\
\hline 10 & $\begin{array}{l}\text { Pottsburg Creek near } \\
\text { South Jacksonville. }\end{array}$ & 9.89 & $1964-66$ & ${ }^{3} 1964$ & $\mathbf{3 0}$ & $\begin{array}{r}10.09 \\
8.95\end{array}$ & $\begin{array}{l}1,740 \\
1,250\end{array}$ & 42 \\
\hline 11 & $\begin{array}{l}\text { Trout River at Dins- } \\
\text { more. }\end{array}$ & 19.9 & $1961-68$ & $\begin{array}{l}=1964 \\
31964\end{array}$ & $\overline{29}$ & $\begin{array}{l}7.13 \\
7.54 \\
9.62\end{array}$ & $\begin{array}{r}564 \\
646 \\
1,140\end{array}$ & $\cdots_{4}$ \\
\hline 12 & $\begin{array}{l}\text { Sixmile Creek near } \\
\text { Marietta. }\end{array}$ & 19.0 & $1964-66$ & ${ }^{3} 1964$ & 30 & $\begin{array}{r}8.63 \\
11.60\end{array}$ & $\begin{array}{l}1,090 \\
2,750\end{array}$ & 41.2 \\
\hline 13 & $\begin{array}{l}\text { Cedar Creek near } \\
\text { Panama Park. }\end{array}$ & 12.0 & $1964-66$ & $\begin{array}{l}21964 \\
331964 \\
3\end{array}$ & $\overline{30}$ & $\begin{array}{l}5.67 \\
5.91 \\
8.39\end{array}$ & $\begin{array}{r}239 \\
282 \\
1,250\end{array}$ & 20 \\
\hline
\end{tabular}

1 Unknown.

2 May; a non-tropical storm.

3 September; Hurricane Dora.

${ }^{4}$ Ratio of peak discharge to 50 -year flood. 
28-31 floods. A comparison of basin characteristics for streams in the Jacksonville area with those of North and South Forks of Black Creek indicated that runoff characteristics were probably similar, particularly with respect to basin and channel slopes. one possible exception is Thomas Creek (No. 1, table 21), head of Nassau River basin, whose characteristics may be more comparable to those of St. Marys River basin, particularly with respect to basin storage.

Recurrence intervals for the August 28-31 flood peaks ranged from about 4 years to more than 50 years. Unit peak discharges ranged from $53 \mathrm{cfs}$ per sq mi to $145 \mathrm{cfs}$ per sq mi (No. 12, table 21). In the southern part of Duval County peak discharges at four stations, $7,8,10$, and 12, averaged $122 \mathrm{cfs}$ per $\mathrm{sq} \mathrm{mi}$; that at Sixmile Creek (No. 12, table 21) was $145 \mathrm{efs}$ per sq mi and 1.2 times the 50-year discharge. Table 21 and figure 26 may be used to compare the $\mathrm{Au}-$ gust 28-31 floods with the two outstanding floods of 1964. Storm rainfall associated with each of the 1964 floods was considerably less than that for the August 28-31 storm. However, soil moisture and general streamflow conditions preceding the 1964 storms were much more conducive to producing floods than antecedent conditions associated with the August floods. For example, cumulative runoff preceding the 1964 floods at long-term station 5 was

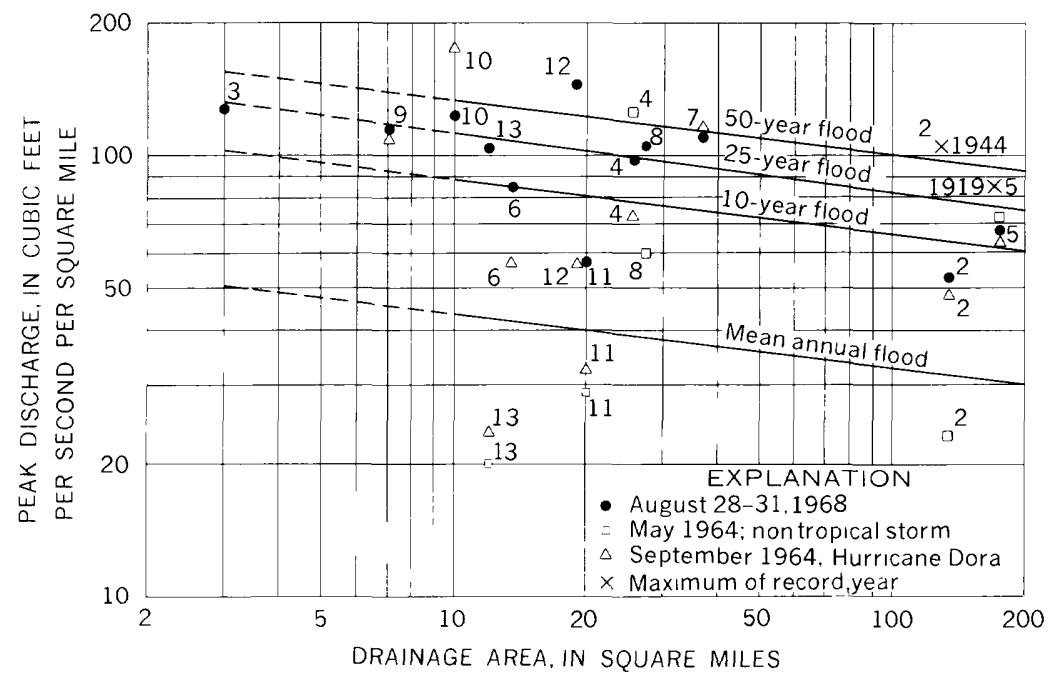

Figure 26.-Relation of August 28-31 flood peaks to major flood peaks of record in the vicinity of Jacksonville, Fla. Numbers correspond to flood determination points in table 21 . 
about four times normal for that time of year, while cumulative runoff preceding the August 1968 floods was only about 11/2 times normal. In fact, the northern and northwestern part of the State was experiencing a moderate drought until the rains in late spring and summer.

Peak stages at four stations, Nos. 2, 7, 8, and 11, ranged from about 1 to 4 feet higher than those of May 1964. Peak stages of August 1968 at six stations, Nos. 2, 4-7, and 9, ranged from about the same to about half a foot higher than those resulting from Hurricane Dora (September 1964); peak stages at three stations, Nos. 11-13, were about 2 to 3 feet higher. At one station, No. 5, the August peak stage was 2 feet lower than the maximum known stage which occurred in 1919 and the peak discharge was only 20 percent less than that of 1919 .

\section{REFERENCES CITED}

Bodhaine, G. L., 1963, Indirect measurement of peak discharge through culverts: U.S. Geol. Survey open-file rep., 49 p.

Corbett, D. M., and others, 1943, Stream-gaging procedure, a manual describing methods and practices of the Geological Survey: U.S. Geol. Survey Water-Supply Paper 888, 245 p.

Cross, W. P., and Webber, E. E., 1959, Floods in Onio, magnitude and frequency: Ohio Dept. Natural Resources, Div. Water Bull. 32, 325 p.

Dalrymple, Tate, and others, 1937, Major Texas floods of 1936: U.S. Geol. Survey Water-Supply Paper 816, $146 \mathrm{p}$.

Gilstrap, R. C., 1972, Floods of May 1968 in south Arkansas: U.S. Geol. Survey Water-Supply Paper 1970-A, 94 p.

Johnson, Hollister, 1936, The New York State flood of July 1935: U.S. Geol. Survey Water-Supply Paper 773-E, p. 233-268.

Kindsvater, C. E., Carter, R. W., and Tracy, H. J., 1953, Computation of peak discharge at contractions: U.S. Geol. Survey Circ. 284, 34 p.

Rostvedt, J. O., 1965, Summary of floods in the United States during 1960: U.S. Geol. Survey Water-Supply Paper $1790-B, 147$ p.

Speer, P. R., and Gamble, C. R., 1965, Magnitude and frequency of floods in the United States-Part 3-A, Ohio River basin except Cumberland and Tennessee River basins: U.S. Geol. Survey Water-Supply Paper $1675,630 \mathrm{p}$.

Tracy, H. J., 1957, Discharge characteristics of broad-crested weirs: U.S. Geol. Survey Circ. 397, 15 p.

U.S. Army Corps of Engineers, 1968a, Post flood report, storm of 5 January 1968, Island of Oahu: Hawaii Division Water and Land Devel. Circ. C49, 15 p.

1968b, Post flood report, storm of 15-16 April 1968, Island of Maui: Hawaii Div. Water and Land Devel. Circ. C50, 20 p.

-Jacksonville District, 1969, Flood plain information, St. Johns River, Jacksonville, Fla.: 71 p.

U.S. Weather Bureau, 1961, Rainfall frequency atlas of the United States: U.S. Weather Bur. Tech. Paper 40, 115 p. 
1962, Rainfall frequency atlas of the Hawaiian Islands for areas to 200 square miles, durations to 24 hours, and return periods from 1 to 100 years: U.S. Weather Bur. Tech. Paper 43, 60 p.

1968, Climatological data (by States): U.S. Weather Bureau, various months, 1968.

Wood, G. K., Swallow, L. A., Johnson, C. G., and Searles, G. H., 1970, Flood of March 1968 in eastern Massachusetts and Rhode Island: U.S. Geological Survey open-file rep., $33 \mathrm{p}$. 


\section{INDEX}

[ Italic numbers indicate major references ]

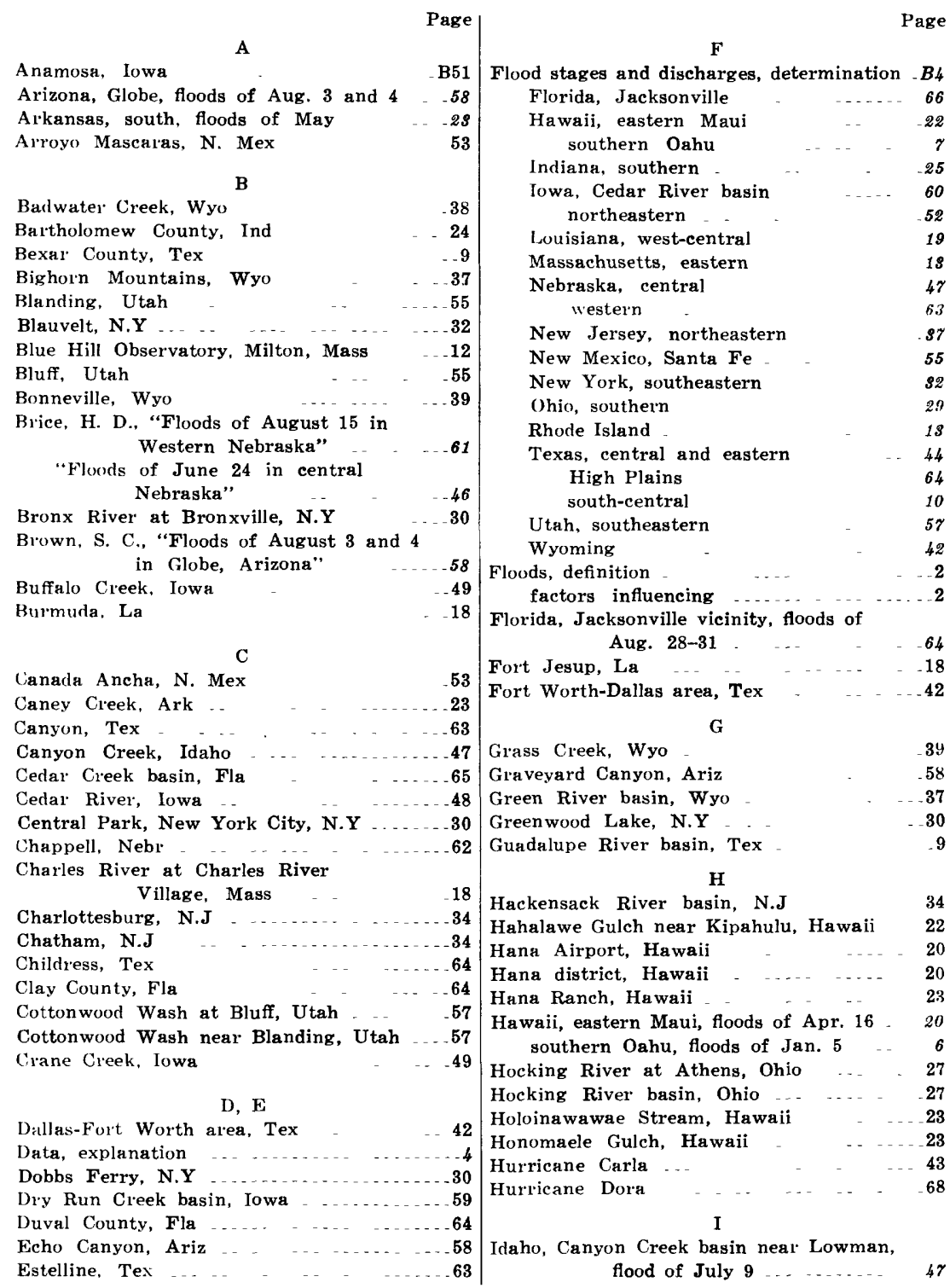


Imeson Airport, Jacksonville, Fla. . . _ _ B65

Independence, Iowa _......... . . . 49

Indiana, southern, floods of May 24-27 _. - 24 Iowa, Cedar River basin, floods of Aug. 5-6 .59 Iowa, northeastern, floods of July 16-20 _..48 Iowa River basin, Iowa _......... . 48 Ipswich River near Ipswich, Mass _..... 12

Jackson County, Ind

$$
\mathbf{J}, \mathbf{K}
$$

Jennings County, Ind . . . . . . . . . . . . . 24

Johnson County, Ind ............... 24

Kaupo Ranch House, Hawaij ...........21

Kawaipapa Gulch at Hana, Hawaii ....... 22

Kipahulu, Hawaii _................. 21

Koolau Range, Hawaii .. . . . . . . . 6

L

Lake Houston, Tex

Laurelville, Ohio .

Lee, F. N., "Floods of April 8-11 in west-central Louisiana"

Little Gooseberry Creek, Wyo

Little Miami River basin, Ohio ......... 27

Little Missouri River, Ark .............23

Little Rock, Ark ..................... 23

Little Wapsipinicon River, Iowa _........49

Lodgepole Creek at Ralton, Nebr ........62

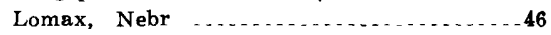

Los Cerros Reservoir, Santa Fe, N. Mex ....53

Louisiana, west-central, floods of

Lowman, Idaho

$$
\text { Apr. 8-11 ........... } 18
$$

\section{M}

Makiki Valley area, Hawaii

Makiki Valley area, Hawaii
Malone Creek at Independence, Iowa $\ldots \ldots .49$

Mamaroneck River at Mamaroneck, N.Y _. . 30

Many, La - . . . . . . . . . . . 18

Many-Robeline area, La .................. 18

Massachusetts, eastern, floods of Mar.

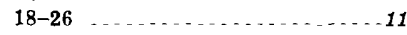

Maui, Hawaii ....................... 20

Montgomery area, La ......................

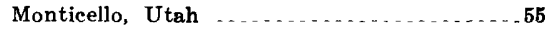

Montezuma Creek, Utah _............. 57

Moomoonui Gulch at Hana, Hawaii ....... 22

Mountian View, Wyo ...................40

\section{$\mathbf{N}$}

Nassau County, N.Y

Nassau River basin, Fla

Natchitoches Parish, La ............... 19

Nebraska, central, floods of June $24 \ldots \ldots .46$

western, floods of Aug. 15 .......61

New Jersey, northeastern, floods of

$$
\text { May 29-31 }
$$

New Mexico, Santa Fe, floods of July 25

New York, southeastern, floods of May

$$
\text { 29-30 }
$$

New York City, N.Y

North Fork Black Creek near Middleburg,

$$
\text { Fla }
$$

Nueces River basin, Tex

\section{o}

Page

Oahu, Hawaii . . . .

O'Connell, D. J., “Floods of June and

August in Wyoming" .........37

Ohio Brush Creek near West Union, Ohio - 27

Ohio, southern, floods of May 24-28 _......27

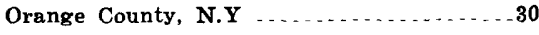

Pacific Palisades Drainageway, Hawaii ... . 9

Paint Creek basin, Ohio ..............27

Palikea Stream below diversion dam near Kipahulu, Hawaii ........22

Parkexsburg, Iowa . . . . . . . . . . . . . 59

Passaic River basin, N.J . . . . . . . . 34

Passaic River near Chatham, N.J _...... 34

Pawcatuck River, R.I _.............. 18

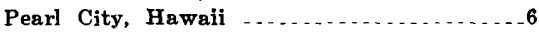

Pearl City Terrace station, Pearl

City, Hawaij $\ldots \ldots \ldots \ldots$

Pinal Creek, Ariz _.................58

Pine Creek, Iowa . . . . . . . . . . . 49

Potowomut River, R.I _................... 18

Prairie Dog Town Fork Red River above

Palo Duro State Park near

Canyon, Tex ..............64

Prairie Dog Town Fork Red River

basin, Tex ................63

Prairie Dog Town Fork Red River near

Canyon, Tex ..............64

Punanani Gulch, Hawaii ................

Pyper, G. E., "Floods of August 1-2

in southeastern Utah"

\section{R}

Rabon, J. W., "Floods of August 28-31 in the vicinity of Jacksonville, Florida"

Racoon Creek at Adamsville, Ohio ...... 27

Racoon Creek basin, Ohio _......... 27

Rahway River basin, N.J _........... 34

Ramapo River near Mahwah, N.J ......31

Ravenna, Nebr ................. 46

Rhode Island, floods of Mar. 18-26 _

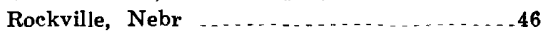

References cited .................68

Robeline-Many area, La _......... 18

Robinson, F. L., "Floods of May 29-30 in southeastern New York" ......so

Rockland County, N.Y ............

\section{$\mathbf{S}$}

Sabine Parish, La _.................... 19

Saddle River basin, N.J _............. 34

St. Marys River basin, Fla ..........67

St. Michael, Nebr ................. 46

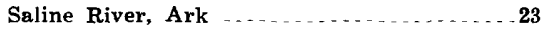

Salt Creek above damsite near

Londonderry, Ohio _. $\ldots 27$

Salt Creek basin, Ohio ...............27

San Antonio, Tex … . . . . . . . . 9

San Jacinto River basin, Tex ....... 42

San Juan River basin, Utah ......... 55

Sangre de Christo Mountains, N. Mex _... 53

Schwob, H. H., "Floods of August 5-6 in

Cedar River basin, Iowa" .... 59 
Schwob, H. H.--Continued

"Flood of July 16-20 in northeastern Iowa""

Shelby County, Ind ........

Shell Rock, Iowa _................ 49

Sixmile Creek near Marietta, Fla ........66

Smackover Creek, Ark _...............23

South Fork Black Creek near Penney

Far'os, Fla.

South Loup River, Nebr _...........46

Stearns, L. G., "Floods of August 28-29 in the Texas High Plains" ...63

"Floods of January 18-24 in south.

Suffern, N.Y central Texas"

Taunton River, Mass

\section{$\mathbf{T}$}

Texas, central and eastern, floods of

Tune 23-28

High Plains, floods of Aug. 28-29 _. _.69

south-central, floods of Jan. 18-24 $\ldots \ldots-9$

Thermopolis-Worland area, Wyo ..........39

Thomas, C. A., "Flood of July 9 in Canyon Creek basin near Lowman, Idaho"

Thomas Creek near Crawford, Fla _.....67

Todd Fork near Roachester, Ohio ........28

Tropical storm Candy _..................42

Twomile Reservoir, N. Mex _...........53

Page

Uinta Mountains, Wyo _...

Ulster County, N.Y $\ldots \ldots \ldots \ldots$

Utah, southeastern, floods of Aug. 1-2 ....55

Virden Creek, Iowa ..................49

$\mathbf{W}, \mathbf{Y}$

Wading River, Mass _.................18

Waiawa, Hawaii _................... 9

Waiawa Stream, Hawaii _.............

Wailuku, Hawaii _. $\ldots \ldots \ldots 22$

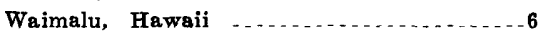

Waimano Flood Channel, Hawaii _......... 7

Wapsipinicon River at Independence,

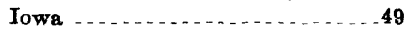

Wapsipinicon River basin, Iowa ........48

Wapsipinicon River near DeWitt, Iowa _. . 49

Waterloo, Iowa _.......... 49

Waverly, Iowa _...

Webber, E. E., "Floods of May 24-28

in southern Ohio" .........27

Westchester County, N.Y ......................

Winn Parish, La ......................

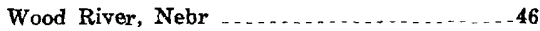

Worland Airport, Wyo _..............39

Worland-Thermopolis area, Wyo .........39

Wyoming, floods of June and August ...... 87

Yellowstone River basin, Wyo _.........37 


\section{Floods of 1968 in the United States}

GEOLOGICALSURVEY WATER-SUPPLYPAPER 1970

This volume was published as separate chapters $A$ and $B$

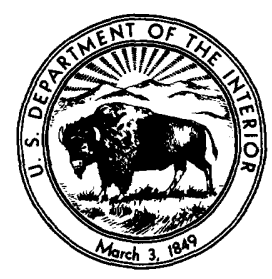


UNITED STATES DEPARTMENT OF THE INTERIOR

ROGERS C. B. MORTON, Secretary

GEOLOGICAL SURVEY

V. E. McKelvey, Director 


\section{CONTENTS}

[Letters designate the separately published chapters]

(A) Floods of May 1968 in south Arkansas, by R. C. Gilstrap.

(B) Summary of floods in the United States during 1968, by J. O. Rostvedt and others. 


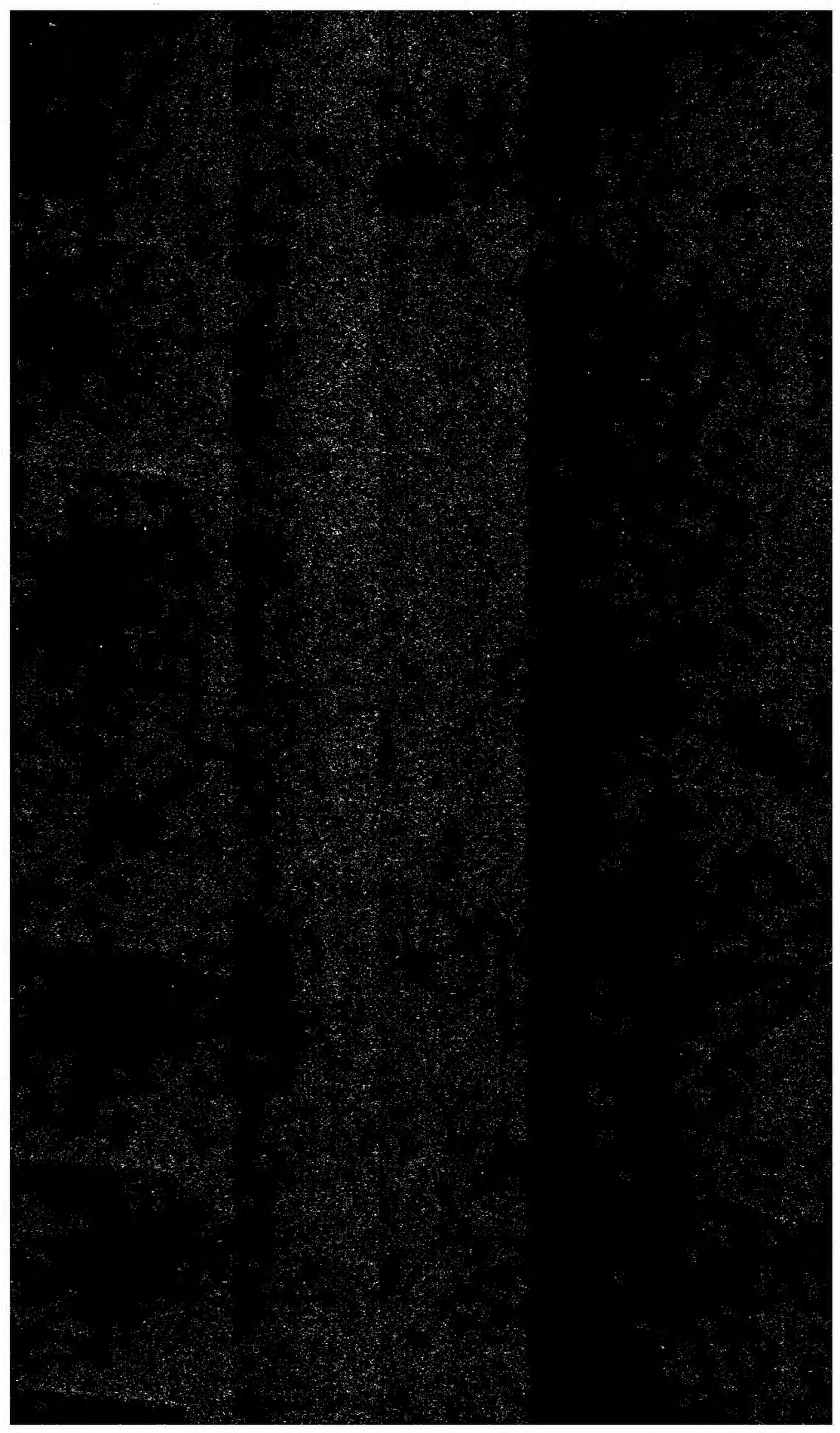

